\title{
HTGR Dust Safety Issues and Needs for Research and Development
}

The INL is a

U.S. Department of Energy National Laboratory

operated by

Battelle Energy Alliance

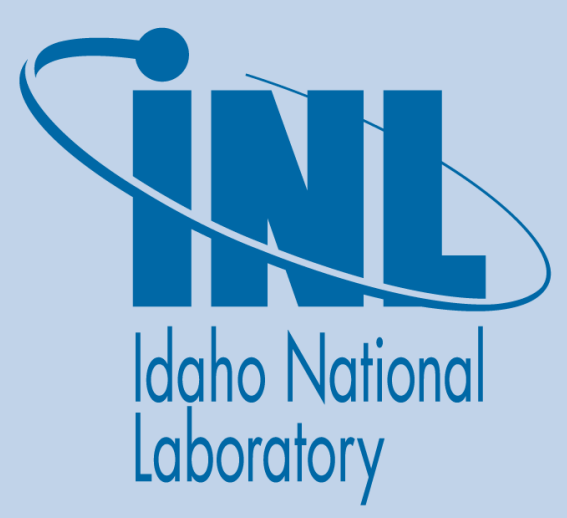

P. W. Humrickhouse

June 2011

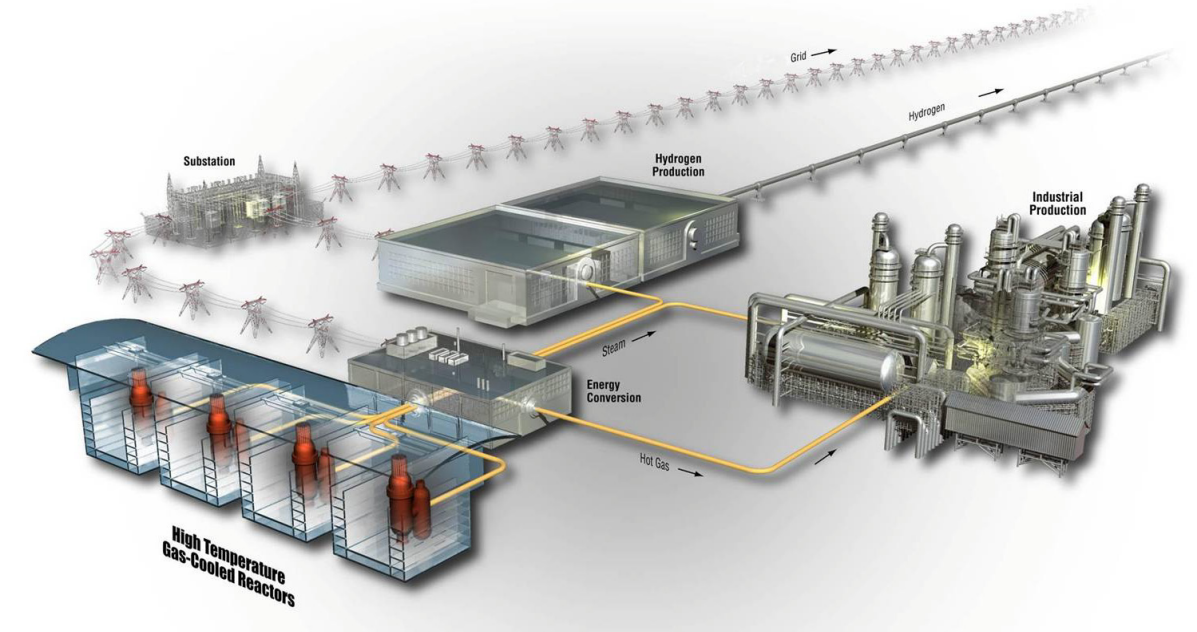




\section{DISCLAIMER}

This information was prepared as an account of work sponsored by an agency of the U.S. Government. Neither the U.S. Government nor any agency thereof, nor any of their employees, makes any warranty, expressed or implied, or assumes any legal liability or responsibility for the accuracy, completeness, or usefulness, of any information, apparatus, product, or process disclosed, or represents that its use would not infringe privately owned rights. References herein to any specific commercial product, process, or service by trade name, trade mark, manufacturer, or otherwise, does not necessarily constitute or imply its endorsement, recommendation, or favoring by the U.S. Government or any agency thereof. The views and opinions of authors expressed herein do not necessarily state or reflect those of the U.S. Government or any agency thereof. 


\title{
HTGR Dust Safety Issues and Needs for Research and Development
}

\author{
P. W. Humrickhouse
}

June 2011

\begin{abstract}
Idaho National Laboratory
Next Generation Nuclear Plant Project

Idaho Falls, Idaho 83415
\end{abstract}

http://www.inl.gov

Prepared for the

U.S. Department of Energy

Office of Nuclear Energy

Under DOE Idaho Operations Office

Contract DE-AC07-05ID14517 

Next Generation Nuclear Plant Project

HTGR Dust Safety Issues and Needs for Research and Development

INL/EXT-11-21097

June 2011

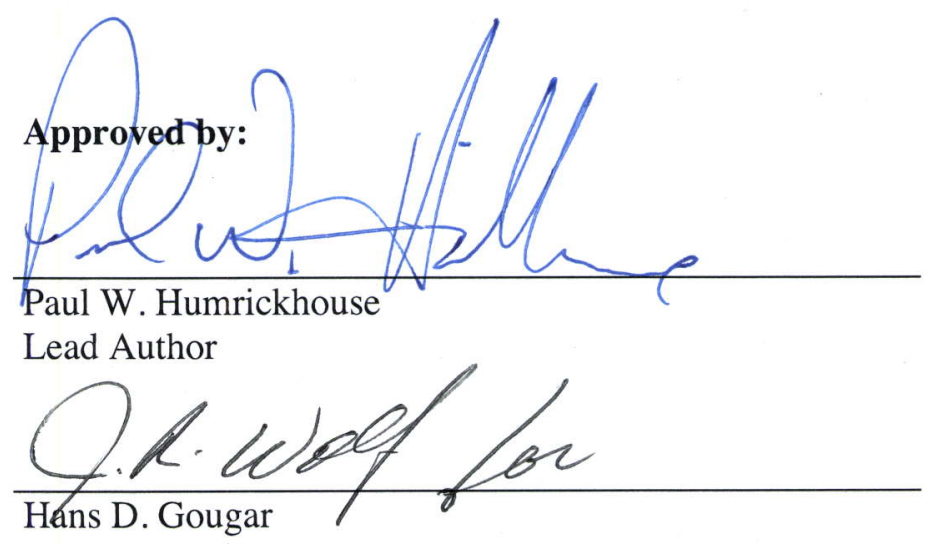

VHTR TDO Technical Deputy Director

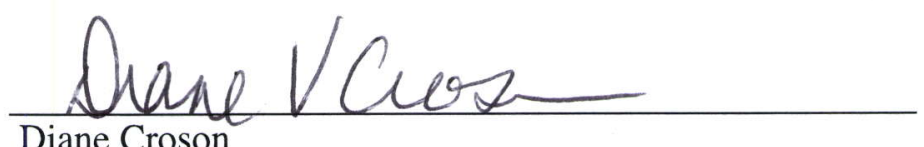

Diane Croon

VHTR TDO Deputy Director
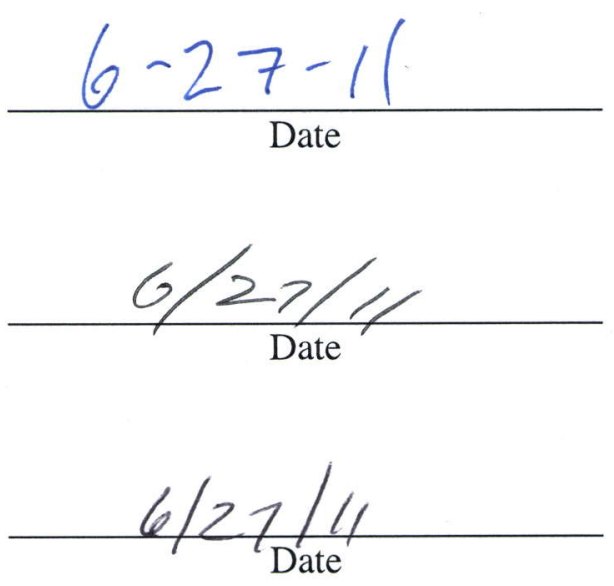



\begin{abstract}
This report presents a summary of high temperature gas-cooled reactor dust safety issues. It draws upon a literature review and the proceedings of the Very High Temperature Reactor Dust Assessment Meeting held in Rockville, MD in March 2011 to identify and prioritize the phenomena and issues that characterize the effect of carbonaceous dust on high temperature reactor safety. It reflects the work and input of approximately 40 participants from the U.S. Department of Energy and its National Labs, the U.S. Nuclear Regulatory Commission, industry, academia, and international nuclear research organizations on the topics of dust generation and characterization, transport, fission product interactions, and chemical reactions. The meeting was organized by the Idaho National Laboratory under the auspices of the Next Generation Nuclear Plant Project, with support from the U.S. Nuclear Regulatory Commission.

Information gleaned from the report and related meetings will be used to enhance the fuel, graphite, and methods technical program plans that guide research and development under the Next Generation Nuclear Plant Project. Based on meeting discussions and presentations, major research and development needs include: generating adsorption isotherms for fission products that display an affinity for dust, investigating the formation and properties of carbonaceous crust on the inside of high temperature reactor coolant pipes, and confirming the predominant source of dust as abrasion between fuel spheres and the fuel handling system.
\end{abstract}




\section{CONTENTS}

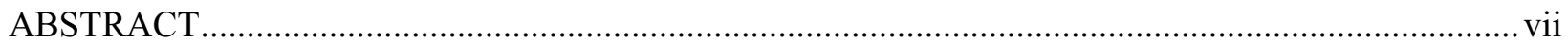

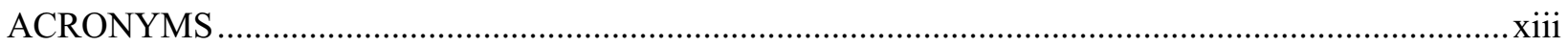

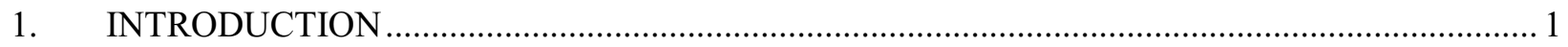

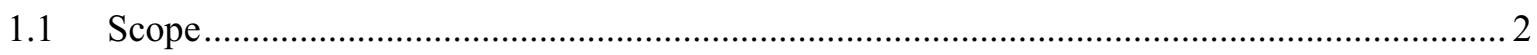

1.2 Outline

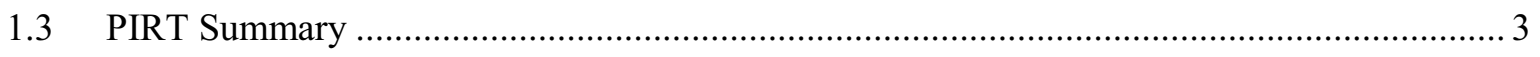

2. DUST GENERATION AND CHARACTERIZATION .............................................................. 6

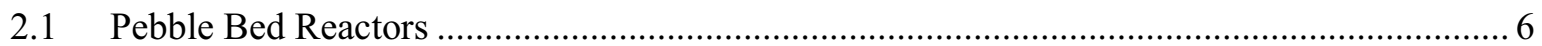

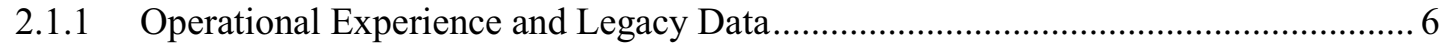

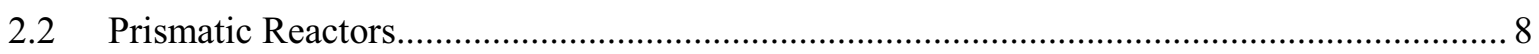

2.2.1 Operational Experience and Legacy Data ......................................................... 8

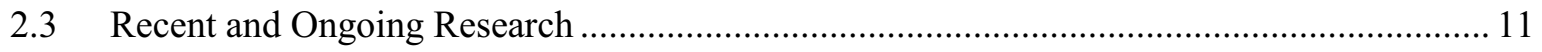

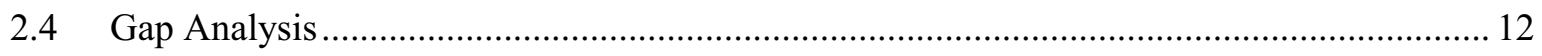

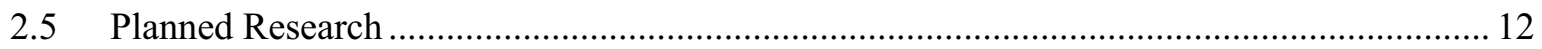

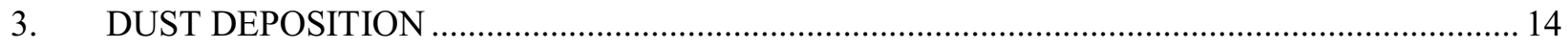

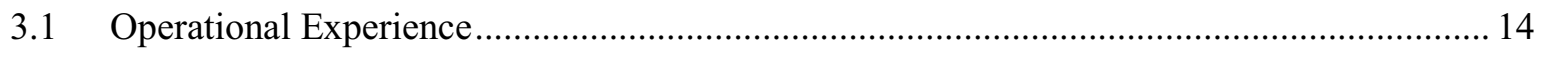

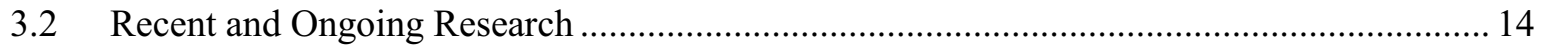

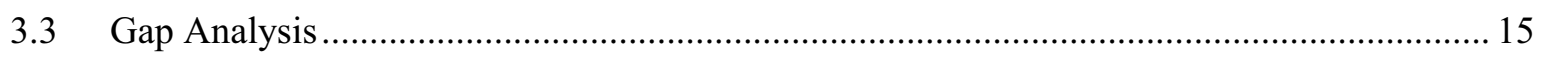

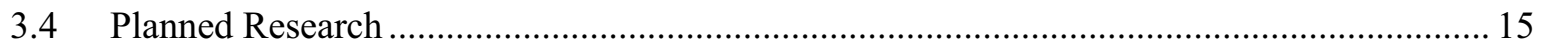

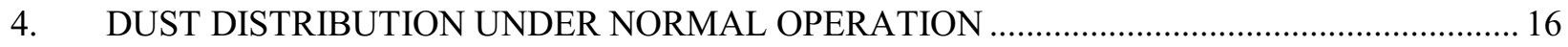

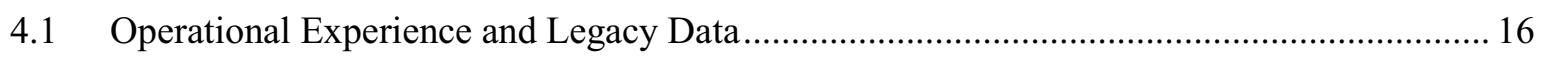

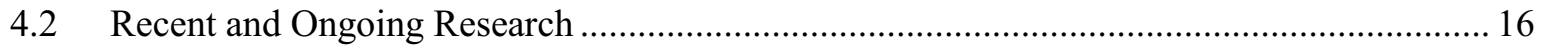

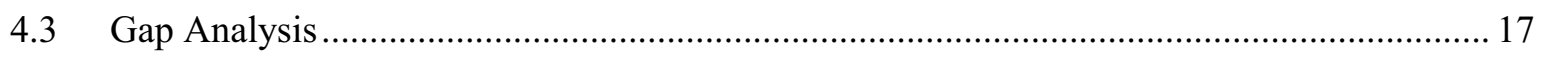

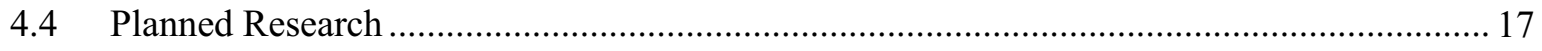

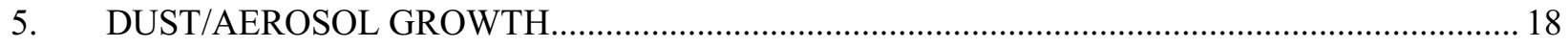

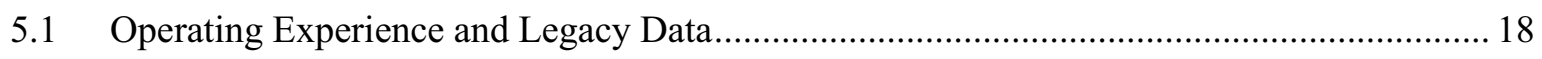

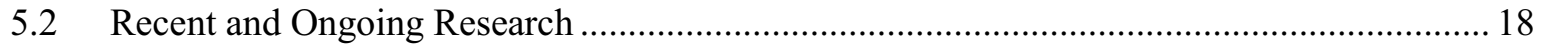

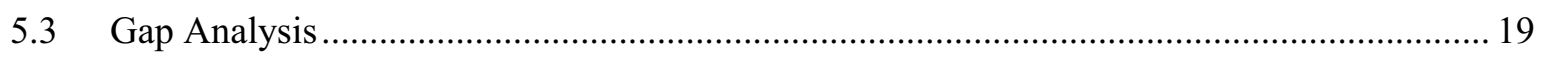

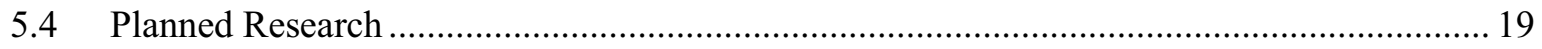

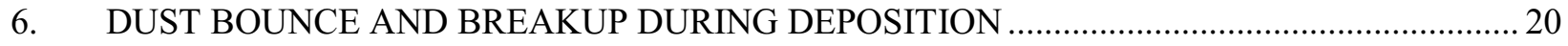

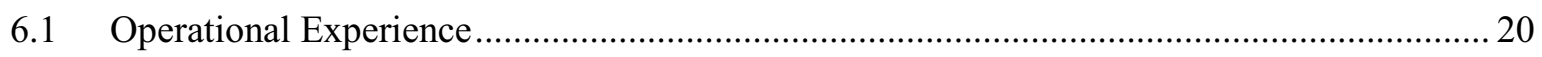

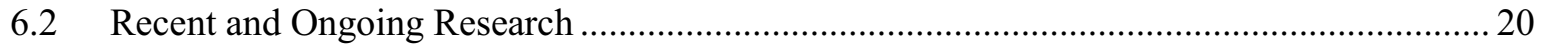

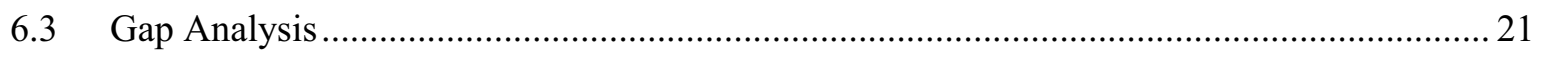

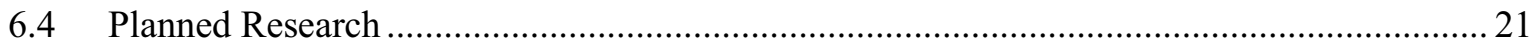

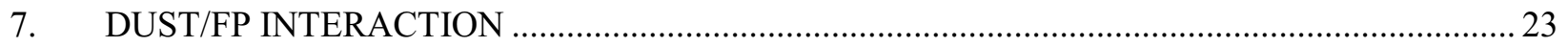




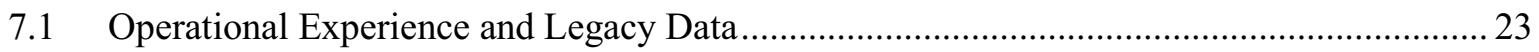

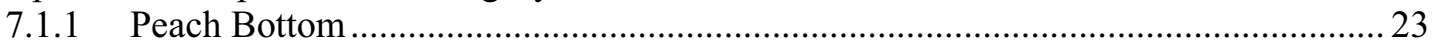

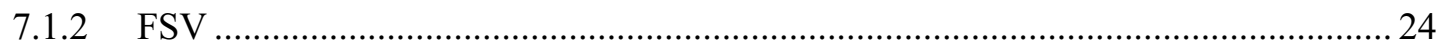

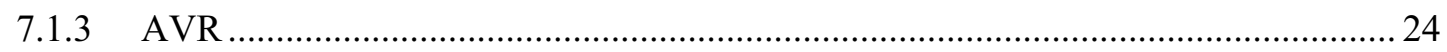

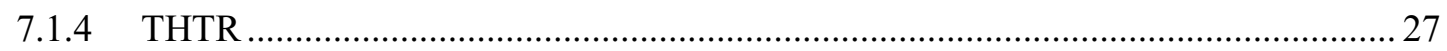

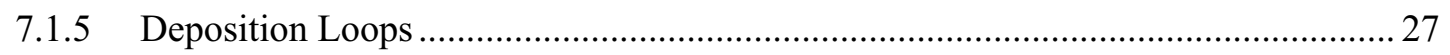

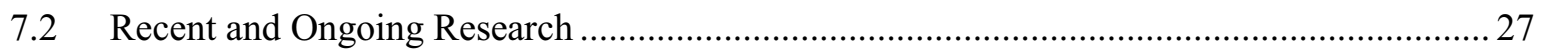

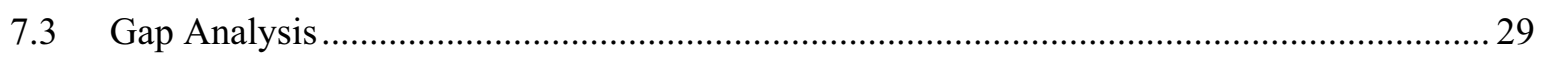

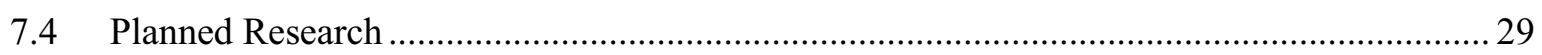

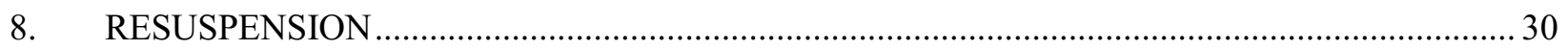

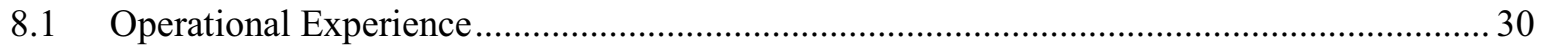

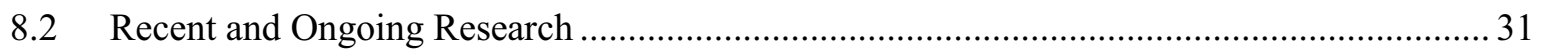

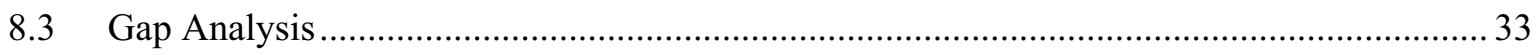

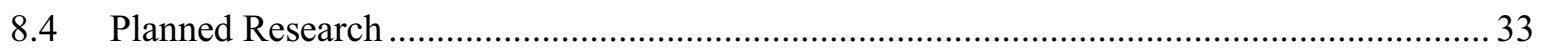

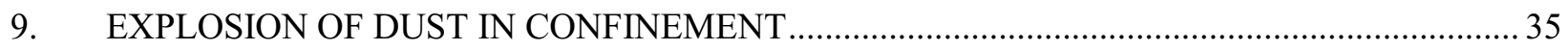

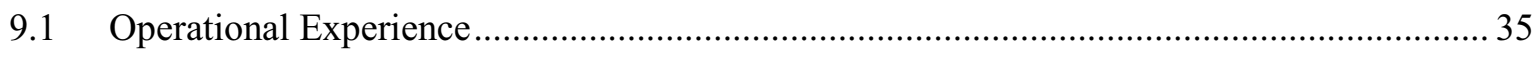

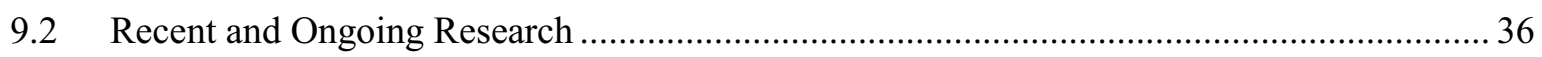

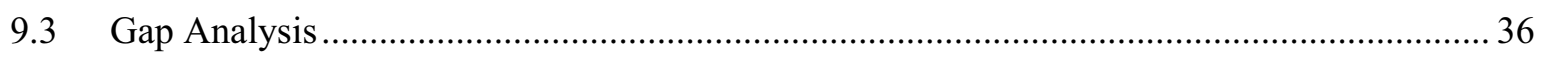

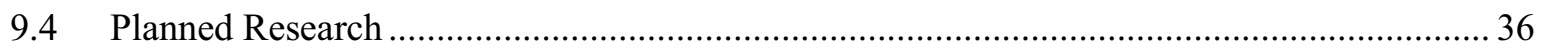

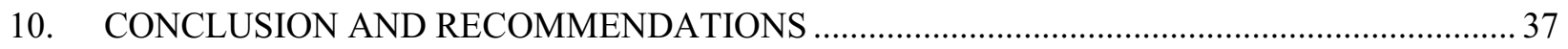

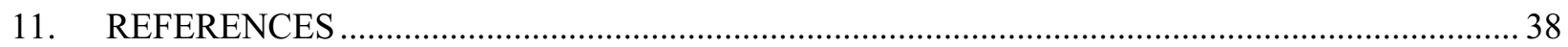

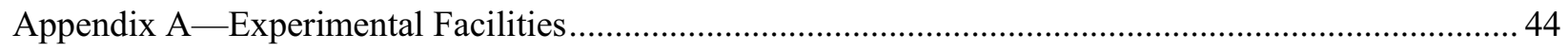

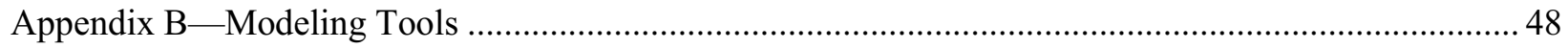

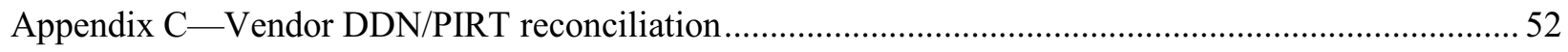

Appendix D_Proceedings of the VHTR Dust Assessment Meeting .........................................................56

\section{FIGURES}

Figure 1. Schematic of AVR and associated experiments (Moorman 2008) ......................................... 7

Figure 2. Dust size distributions in AVR (left [IAEA 1997]; right [von der Decken \& Wawrzik 1990]).

Figure 3. Size distribution of fine Peach Bottom dust (Dyer, Wichner, Martin, \& de Nordwall 1977). 10

Figure 4. Particle size distribution as a function of time in the University of Missouri experiment. ......... 12

Figure 5. Circulating dust concentrations in AVR (Moorman 2008)...................................................... 16

Figure 6. Dynamic shape factor as a function of particles size, from (NEA 2009).................................. 18 
Figure 7. Probability of fly ash particle bounce as a function of kinetic energy (D. Powers, Dust Issues Assessment Meeting).

Figure 8. Circulating dust concentrations in AVR (Moorman 2008). ....................................................25

Figure 9. Circulating dust concentrations in the AVR resuspension experiments.................................. 30

Figure 10. Removal of dust by scraping a section of AVR pipe (Fachinger et al. 2008)........................ 31

Figure 11. ECART correlation for the resuspension rate and experiment data (Parozzi 1997)................. 32

\section{TABLES}

Table 1. Dust issues extracted from Table 10 of the NGNP FP transport PIRT (NRC 2008).................... 3

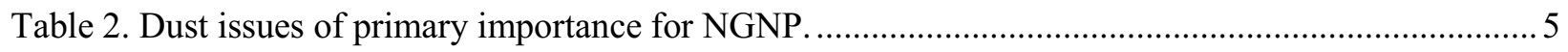

Table 3. Specific activity of Peach Bottom Dust as a function of particle size Bottom (Dyer, Wichner, Martin, \& de Nordwall 1977).

Table 4. Specific activity of soot-like dust in Peach Bottom (Dyer, Wichner, Martin, \& de

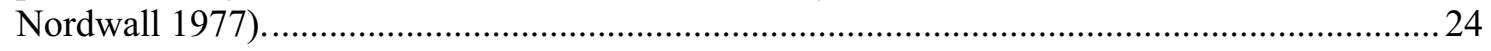

Table 5. Results of AVR dust experiments (IAEA 1997) ...................................................................25

Table 6. Other dust specific activities from various locations in AVR (IAEA 1997).............................26 


\section{ACRONYMS}

AVR Arbeitsgemeinschaft Versuchsreaktor (Germany; Association Experimental Reactor Ltd.)

BET Brunauer-Emmett-Teller

CFD computational fluid dynamics

DDN Design Data Need

FHS fuel handling system

FP fission product

FSV Fort St. Vrain

HTGR high temperature gas-cooled reactor

HTHL high temperature helium loop

HTR high temperature reactor

HTTR High Temperature Engineering Test Reactor

INET Institute for Nuclear Energy Technology

INL Idaho National Laboratory

JAEA Japan Atomic Energy Agency

LWR light water reactor

NGNP Next Generation Nuclear Plant

NRC Nuclear Regulatory Commission

PBMR Pebble Bed Modular Reactor

PIRT Phenomena Identification and Ranking Table

RCCS reactor cavity cooling system

$\mathrm{R} \& \mathrm{D} \quad$ research and development

SNL Sandia National Laboratory

THTR Thorium Hochtemperatur Reaktor

TRISO tristructural isotropic

VHTR very high temperature reactor 


\section{INTRODUCTION}

Though the design of tristructural isotropic (TRISO) fuel for high temperature gas-cooled reactors (HTGRs) will prevent large-scale failure and fission product release, even in accident scenarios, radiologically significant quantities of fission products will be present in the primary system. Some result from a very small fraction of initially defective fuel particles, while others may be released from intact fuel because of diffusion at very high temperatures. Uranium contamination outside the fuel particles is also a source of fission products.

Following release from fuel during normal operation, fission products will be transported in the coolant gas, and plateout onto surfaces in the primary system. This distribution of circulating and deposited activity is important for estimating maintenance doses and establishes the initial condition of an accident scenario. The high-pressure coolant provides a mechanism to transport fission products from the primary system to the environment in the event of a breach of the reactor coolant system (RCS). Research into dust issues within the primary loop is characterized largely by the study of graphite component degradation and interactions with fission products. Outside the primary system (but within the reactor building), research is focused on thermal fluid transport and the retention of dust within large structures.

Analysis of the fission product distribution, in both normal and accident scenarios, is complicated in an HTGR by the likely presence of dust. Tens to hundreds of kilograms of dust have been generated within operating high temperature reactors via a variety of physical phenomena. In pebble bed reactors, the primary generation mechanism is assumed to be friction between circulating pebbles; the resulting material is carbonaceous. Lacking that generation mechanism, a prismatic reactor of comparable size and operating history would be expected to contain far less dust.

Dust complicates the prediction of fission product (FP) transport since it presents a parallel transport path for FPs. In the absence of dust, the following general transport path can be envisioned:

1. Release from fuel and transport through the graphite fuel element (prismatic) or fuel matrix (pebble bed)

2. Transport as a vapor in the primary coolant

3. Plate-out by adsorption on RCS surfaces

4. Lift off in accident flow transients

5. Subsequent transport in the gas phase dependent on the scenario.

A similar path can be outlined for dust particles generated in the core, transported in the coolant, deposited on surfaces, and resuspended and transported during flow transients. However, the mechanisms governing each step of the process are different for solid particles than for gaseous FPs. The difficulty arises when FPs interact with (adsorb on/adhere to) dust particles, which may occur where both circulate in the coolant or where both co-deposit on surfaces. To the extent that this occurs, FP transport will then be governed by dust transport.

When dispersed in a gas, the nominally micron-sized dust particles constitute an aerosol. Thus, many of the models and methods of aerosol transport, such as those applied to light water reactor (LWR) FP aerosols, apply. There are, however, important differences. The generation mechanism is clearly one of these; an HTGR aerosol comprises graphite particles generated by friction, rather than, for example, nucleation of FP vapors. The properties of the constituent particles, then, can be expected to be rather different. The absence of water or steam in most HTGR accident scenarios is another important difference that may affect various transport phenomena. 
A Phenomena Identification and Ranking Table (PIRT) exercise was conducted for issues related to FP transport in general. A subset of these are related to dust, and these formed the basis for a more indepth consideration of specific dust issues. To that end, a Very High Temperature Reactor Dust Assessment Meeting was held in Rockville, MD during March 2011 to identify and prioritize these phenomena. The meeting included approximately 40 participants from the U.S. Department of Energy and its National Labs, the U.S. Nuclear Regulatory Commission (NRC), industry, academia, and international nuclear research organizations. It was organized by Idaho National Laboratory under the auspices of the Next Generation Nuclear Plant (NGNP) Project, with support from the U.S. Nuclear Regulatory Commission.

This resulting document attempts to capture the work and thoughts of the participants in the meeting on the topics of dust generation and characterization, transport, FP interactions, and chemical reactions. Information gleaned from the report and meeting will be used to enhance the fuel, graphite, and methods technical program plans that guide research and development (R\&D) under the NGNP Project.

\subsection{Scope}

Many issues related to NGNP safety in general, and FP behavior in particular, are unrelated to dust. PIRT documents (NRC 2008), gap analyses (Ball 2008), and many other issue summaries related to gas reactors or NGNP and FP transport can be found in the literature (cf. the references of this report). Understanding many of these issues is a prerequisite to any meaningful NGNP safety analysis, regardless of the specific design and with or without the presence of dust. For example, the importance of dust as a carrier of FPs obviously depends on the FP release from fuel. Nevertheless, this work does not seek to address such issues. The focus here is exclusively on issues related to dust.

Furthermore, this study is primarily restricted to issues related to the source term. Other potential harmful effects of dust not considered in any detail here include fouling of heat transfer surfaces, instrument line plugging, and carburization of metals above $850^{\circ} \mathrm{C}$ (Hanson 2008). Throughout, it will be apparent that the preferred accident modeling tools are system codes such as MELCOR, SPECTRA, DAMD, ASTEC, RELAP, etc. The first three have been or are being developed for gas reactors, and as such are the focus of the discussion. Of these, MELCOR will be the most frequently cited example, as it is available to (and more familiar to) the author.

\subsection{Outline}

The remainder of the report is organized as follows:

- Identification of relevant dust issues from the NGNP PIRT.

- Discussion of each issue, including:

- A review of HTGR operating experience with respect to dust and summary of available legacy data

- A review of recent and ongoing research on each dust issue

- Identification of knowledge gaps.

- Conclusions and recommendations.

Vendor responses to the PIRT dust issues, summaries of codes and operating experiment facilities related to the HTGR dust issue, and notes from the very high temperature reactor (VHTR) dust issues assessment meeting are provided in the appendixes. 


\subsection{PIRT Summary}

Safety-relevant phenomena for NGNP have been previously identified in a six-volume PIRT (NRC 2008). Volume 3 of this document focuses on issues related to FP transport, a subset that is related to dust. This section summarizes these dust issues from the PIRT and their knowledge and importance rankings. A total of 56 issues/phenomena were identified in the FP PIRT, and the importance and knowledge level of each was ranked from 1 (low) to 5 (high) by each of five panelists. Sixteen of these issues are directly related or applicable to dust. This subset of 16 issues from the original PIRT table is reproduced in Table 1.

Table 1. Dust issues extracted from Table 10 of the NGNP FP transport PIRT (NRC 2008).

\begin{tabular}{|c|c|c|c|c|c|c|}
\hline ID & $\begin{array}{c}\text { Issue } \\
\text { (Phenomena, } \\
\text { Process, } \\
\text { Geometry } \\
\text { Condition) } \\
\end{array}$ & $\begin{array}{l}\text { Importance } \\
\text { for NGNP } \\
\text { (High, } \\
\text { Medium, } \\
\text { Low) }\end{array}$ & Rationale & $\begin{array}{l}\text { Level of } \\
\text { Knowledge } \\
\text { (High, } \\
\text { Medium, } \\
\text { Low) }\end{array}$ & Rationale & $\begin{array}{c}\text { Status of FP } \\
\text { Modeling } \\
\text { (Adequate, } \\
\text { Minor Mod, } \\
\text { Major Need) }\end{array}$ \\
\hline 9 & $\begin{array}{l}\text { FP plate-out } \\
\text { and dust } \\
\text { distribution } \\
\text { under normal } \\
\text { operation }\end{array}$ & 5-High & Starting conditions & $\begin{array}{l}\text { 1-Low, } \\
\text { 4-Medium }\end{array}$ & $\begin{array}{l}\text { Theory and models lack } \\
\text { specifics }\end{array}$ & Major need \\
\hline 18 & $\begin{array}{l}\text { Sorptivity } \\
\text { graphite }\end{array}$ & 5-High & $\begin{array}{l}\text { Can determine holdup } \\
\text { and release of FP (IC } \\
\text { and Trans.) }\end{array}$ & 5-Medium & $\begin{array}{l}\text { Historical data, need } \\
\text { specific information on } \\
\text { graphite and radiation } \\
\text { effects }\end{array}$ & Minor mod \\
\hline 26 & $\begin{array}{l}\text { Dust } \\
\text { generation }\end{array}$ & 5-High & $\begin{array}{l}\text { Vector for FP } \\
\text { transport; possibility } \\
\text { of high mobility }\end{array}$ & 5-Medium & $\begin{array}{l}\text { Limited experience; lack } \\
\text { specific system } \\
\text { information }\end{array}$ & $\begin{array}{l}\text { Major need, } \\
\text { import from } \\
\text { other groups }\end{array}$ \\
\hline 27 & $\begin{array}{l}\text { (De)Absorptio } \\
n \text { on dust }\end{array}$ & 5-High & $\begin{array}{l}\text { Provides copious } \\
\text { surface area for FP } \\
\text { absorption }\end{array}$ & $\begin{array}{l}\text { 2-Low, } \\
\text { 3-Medium }\end{array}$ & $\begin{array}{l}\text { Limited experience, lack } \\
\text { specific details }\end{array}$ & Major need \\
\hline 32 & $\begin{array}{l}\text { Aerosol } \\
\text { growth }\end{array}$ & $\begin{array}{l}\text { 1-Medium, } \\
\text { 4-High }\end{array}$ & $\begin{array}{l}\text { Low concentration } \\
\text { growth can lead to } \\
\text { high shape factors and } \\
\text { unusual size } \\
\text { distribution }\end{array}$ & 5-Low & $\begin{array}{l}\text { Regime has not been } \\
\text { studied previously }\end{array}$ & Major need \\
\hline 33 & $\begin{array}{l}\text { Surface } \\
\text { roughness }\end{array}$ & 5-Medium & $\begin{array}{l}\text { Affects aerosol } \\
\text { deposition } 1-5 \text { micron } \\
\text { particles (IC and } \\
\text { Trans.) }\end{array}$ & 5-Medium & $\begin{array}{l}\text { Initial information from } \\
\text { manufacturer; evolution } \\
\text { of surface roughness } \\
\text { during operation not } \\
\text { well known }\end{array}$ & Minor mod \\
\hline 36 & $\begin{array}{l}\text { Aerosol/dust } \\
\text { deposition }\end{array}$ & 5-High & $\begin{array}{l}\text { Gravitational, inertial, } \\
\text { thermophoresis, } \\
\text { electrostatic, } \\
\text { diffusional, } \\
\text { turbophoresis (Trans.) }\end{array}$ & 5-Medium & $\begin{array}{l}\text { Reasonably well } \\
\text { developed theory of } \\
\text { aerosol deposition by } \\
\text { most mechanisms } \\
\text { except inertial impact in } \\
\text { complex geometries; } \\
\text { applicability to NGNP } \\
\text { unclear }\end{array}$ & Minor mod \\
\hline
\end{tabular}




\begin{tabular}{|c|c|c|c|c|c|c|}
\hline ID & $\begin{array}{c}\text { Issue } \\
\text { (Phenomena, } \\
\text { Process, } \\
\text { Geometry } \\
\text { Condition) } \\
\end{array}$ & $\begin{array}{l}\text { Importance } \\
\text { for NGNP } \\
\text { (High, } \\
\text { Medium, } \\
\text { Low) }\end{array}$ & Rationale & $\begin{array}{l}\text { Level of } \\
\text { Knowledge } \\
\text { (High, } \\
\text { Medium, } \\
\text { Low) } \\
\end{array}$ & Rationale & $\begin{array}{c}\text { Status of FP } \\
\text { Modeling } \\
\text { (Adequate, } \\
\text { Minor Mod, } \\
\text { Major Need) }\end{array}$ \\
\hline 37 & $\begin{array}{l}\text { Aerosol/dust } \\
\text { bounce, } \\
\text { breakup } \\
\text { during } \\
\text { deposition }\end{array}$ & $\begin{array}{l}\text { 4-Medium, } \\
\text { 1-High }\end{array}$ & $\begin{array}{l}\text { Can modify deposition } \\
\text { profile and suspended } \\
\text { aerosol distribution }\end{array}$ & $\begin{array}{l}\text { 4-Low, } \\
\text { 1-Medium }\end{array}$ & $\begin{array}{l}\text { Theory, data, and } \\
\text { models lacking }\end{array}$ & Major need \\
\hline 38 & Resuspension & 5-High & $\begin{array}{l}\text { Flow/vibration } \\
\text { induced, saltation; } \\
\text { mechanical forces can } \\
\text { release FPs from pipe } \\
\text { surface layers/films } \\
\text { (Trans.) }\end{array}$ & $\begin{array}{l}\text { 4-Low, } \\
\text { 1-Medium }\end{array}$ & $\begin{array}{l}\text { Lack of data and models } \\
\text { for anticipated } \\
\text { conditions }\end{array}$ & Major need \\
\hline 39 & $\begin{array}{l}\text { Confinement } \\
\text { aerosol } \\
\text { physics }\end{array}$ & 5-High & $\begin{array}{l}\text { Analogous to LWR } \\
\text { aerosol } \\
\text { behavior/physics, } \\
\text { delta-T, chemistry; } \\
\text { important holdup } \\
\text { mechanism (Trans.) }\end{array}$ & 5-Medium & $\begin{array}{l}\text { Reasonably well } \\
\text { developed theory of } \\
\text { aerosol behavior; } \\
\text { applicability to NGNP } \\
\text { unclear }\end{array}$ & Minor mod \\
\hline 40 & $\begin{array}{l}\text { Dust } \\
\text { deposition on } \\
\text { vessel and } \\
\text { RCCS } \\
\text { hardware }\end{array}$ & 5-Low & $\begin{array}{l}\text { Not important for FP } \\
\text { transport but may } \\
\text { affect radiative heat } \\
\text { transfer in reactor } \\
\text { cavity }\end{array}$ & 5-Low & Very limited data & Major need \\
\hline 41 & $\begin{array}{l}\text { Corrosion } \\
\text { products }\end{array}$ & 5-Low & $\begin{array}{l}\text { Spalled surface films; } \\
\text { low corrosion } \\
\text { environment } \\
\text { (IC and Trans.) }\end{array}$ & 5-High & Past experience & Adequate \\
\hline 42 & $\begin{array}{l}\text { Erosion } \\
\text { products, } \\
\text { noncarbon }\end{array}$ & 5-Low & $\begin{array}{l}\text { Low concentration of } \\
\text { course materials } \\
\text { (IC and Trans.) }\end{array}$ & 5-Low & $\begin{array}{l}\text { Lack of design } \\
\text { information; } \\
\text { configuration and } \\
\text { materials specific }\end{array}$ & Adequate \\
\hline 43 & Wash-off & 5-High & $\begin{array}{l}\text { If credible source of } \\
\text { water present; design } \\
\text { dependent (Trans.) }\end{array}$ & 5-Medium & $\begin{array}{l}\text { Some experimental data } \\
\text { available }\end{array}$ & Minor mod \\
\hline 46 & Filtration & 5-High & $\begin{array}{l}\text { Traditional passive } \\
\text { charcoal/ high } \\
\text { efficiency particulate } \\
\text { air (HEPA) filter } \\
\text { (Trans.) }\end{array}$ & 5-High & Historical experience & Adequate \\
\hline 48 & $\begin{array}{l}\text { Combustion } \\
\text { of dust in } \\
\text { confinement }\end{array}$ & 5-High & $\begin{array}{l}\text { Source of heat and } \\
\text { distribution of FPs } \\
\text { with in confinement }\end{array}$ & $\begin{array}{l}\text { 1-Low, } \\
\text { 4-Medium }\end{array}$ & ITER data & Major need \\
\hline
\end{tabular}

Of the 16 issues in the table above, several do not warrant further discussion because of low importance or high knowledge, or may be combined with other issues in the table. Four have either a low importance or high level of knowledge: filtration (46), erosion products, corrosion products, and dust deposition on vessel and reactor cavity cooling system (RCCS) hardware. Some issues may apply to both FPs and dust: wash-off (43) is one such example. It can be assumed (conservatively) that dust will be 
easily removed from surfaces in the event of water ingress, much more so than FPs adsorbed on solid surfaces. Thus, greater knowledge of this issue is less important for dust than for FPs. Similarly, sorptivity on graphite (18) is assumed to refer to sorptivity of FPs on solid graphite surfaces. It is included for completeness above, as the physics of FP sorption on graphite may apply also to graphite dust, or surfaces that are covered with it, even though the surface morphology is much different in this case. This issue will be combined with (De)Absorption on dust (27), or referred to more broadly below as "dust-FP interaction." Surface roughness (33) affects not only dust deposition, but is a governing factor in dust resuspension (38); the two are grouped together in subsequent discussions. Finally, Confinement aerosol physics (39) is presumably governed by the individual phenomena already listed in the table, and is not retained as a separate issue in the following discussion.

The eight remaining dust issues of primary importance for NGNP are summarized in Table 2. A section is devoted to each in what follows. Legacy data, recent and ongoing research, and remaining knowledge gaps related to each issue are discussed in each subsequent section.

These conclusions must be addressed in the context of the overall NGNP R\&D program. A 'Need' is 'Major' relative to the other dust issues identified. The importance and priority relative to other R\&D needs (in fuels, graphite, alloys, and methods) is not prescribed here. In particular, as the impact of dust on HTGR safety arises only as a potential vector for FP transport, the impetus for further dust research must be assessed relative to the gaps in the ability to model HTGR source terms.

Table 2. Dust issues of primary importance for NGNP.

\begin{tabular}{|llll|}
\hline \multicolumn{1}{c}{ Issue } & \multicolumn{1}{c|}{ Importance } & \multicolumn{1}{c|}{ Knowledge level } & \multicolumn{1}{c|}{ Status } \\
\hline Dust distribution under normal operation & 5-High & 1-Low, 4-Medium & Major need \\
Dust generation & 5-High & 5-Medium & Major need \\
(De)Absorption on dust & 5-High & 2-Low, 3-Medium & Major need \\
Aerosol growth & 1-Medium, 4-High & 5-Low & Major need \\
Aerosol/dust deposition & 5-High & 5-Medium & Minor mod \\
Aerosol/dust bounce, breakup during & 4-Medium, 1-High & 4-Low, 1-Medium & Major need \\
deposition & & & \\
Resuspension & 5-High & 4-Low, 1-Medium & Major need \\
Combustion of dust in confinement & 5-High & 1-Low, 4-Medium & Major need \\
\hline
\end{tabular}




\section{DUST GENERATION AND CHARACTERIZATION}

\subsection{Pebble Bed Reactors}

Dust generation in pebble bed reactors is presumed to result primarily from friction, both between pebbles within the core, and between pebbles and the fuel handling system. The latter may contribute a significant portion of the total dust to be found in such a reactor.

\subsubsection{Operational Experience and Legacy Data}

Estimates of the quantity of dust to be expected in a future pebble bed reactor vary and are largely based on scaled estimates of Arbeitsgemeinschaft Versuchsreaktor (AVR; German Association Experimental Reactor Ltd.) dust production.

If one simply scales the AVR dust production rate (revised upward to $\sim 5 \mathrm{~kg} / \mathrm{y}$ by (Moorman 2008)) linearly with reactor power, a $400 \mathrm{MW}$ pebble bed reactor would be expected to generate $\sim 43 \mathrm{~kg} / \mathrm{y}$ of dust (Kissane, 2009). An estimate of $15 \mathrm{~kg} / \mathrm{y}$ dust production has been made in the PBMR SAR (PBMR). This is based on an estimated dust generation rate of $10 \mathrm{mg}$ per pebble per pass, adopted from a Siemens prediction for High Temperature Reactor (HTR)-Modul, based in turn on AVR experience. Other estimates are as high as $100 \mathrm{~kg} / \mathrm{yr}$ (Moorman 2008). This includes abrasion between pebbles in the core itself as well as abrasion between pebbles and the components of the fuel handling system. Results from a series of tests performed on AVR indicate that the latter was the most significant source of dust in AVR (Hittner 2011). This is an important issue to resolve. If significant amounts of dust are generated only between pebble matrix and the fuel handling system, design measures can be taken to mitigate dust generation.

\subsubsection{AVR (Germany)}

The German AVR was a $46 \mathrm{MW}(\mathrm{t})(15 \mathrm{MW}(\mathrm{e}))$ prototype pebble bed reactor that operated for 21 years until 1988. Over that time, it operated using a wide variety of fuel types. Rather large FP inventories resulting from operation with BISO fuel at $950^{\circ} \mathrm{C}$ outlet temperatures and the presence of dust have complicated the decommissioning and decontamination of AVR. During its operation, several experiments related to FP and dust transport were conducted, from which considerable information is available. These include the VAMPYR-I and VAMPYR-II plateout experiments and the cold gas filter experiment shown in Figure 1. Details of the experiment described in this section are largely taken from (IAEA 1997), though discussions can also be found in (EPRI 2002; Gottaut \& Kruger 1990; von der Decken \& Wawrzik 1990; and Moorman 2008).

The size distribution of AVR dust has been measured and was found to largely contain submicron particles with a mean diameter of $0.6 \mu \mathrm{m}$, corresponding to a volume-weighted mean of 5 to $10 \mu \mathrm{m}$. The small size of particles is cited as a reason for the aforementioned small deposition per pass; the size distribution did not appear to vary significantly with the location of the sample. Examples of measured AVR dust size distributions are shown in Figure 2.

Though the consensus at the Dust Assessment Meeting was that friction is indeed the primary dust generation mechanism, it was also proposed that AVR dust was generated by chemical reactions such as precipitation of carbon (Nieder 1990) defined by

$$
\begin{aligned}
& \mathrm{C}+\mathrm{H}_{2} \mathrm{O} \rightleftharpoons \mathrm{H}_{2}+\mathrm{CO} \\
& \mathrm{C}+\mathrm{CO}_{2} \rightleftharpoons 2 \mathrm{CO}
\end{aligned}
$$




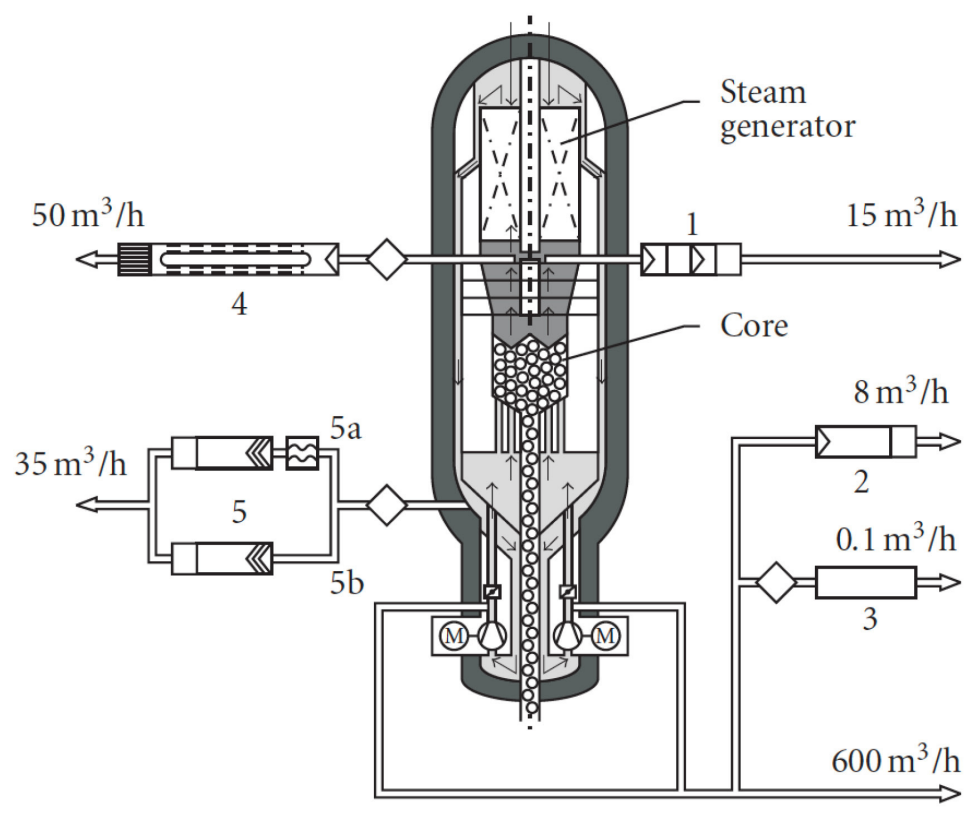

1 VAMPYR I (hot gas filter)

2 Cold gas filter

3 Noble gas measurement

4 VAMPYR II (plate-out experiment)

5 Dust experiment

5a With plate-out section upflow of the dust filters

$5 b$ Without plate-out section

Figure 1. Schematic of AVR and associated experiments (Moorman 2008).
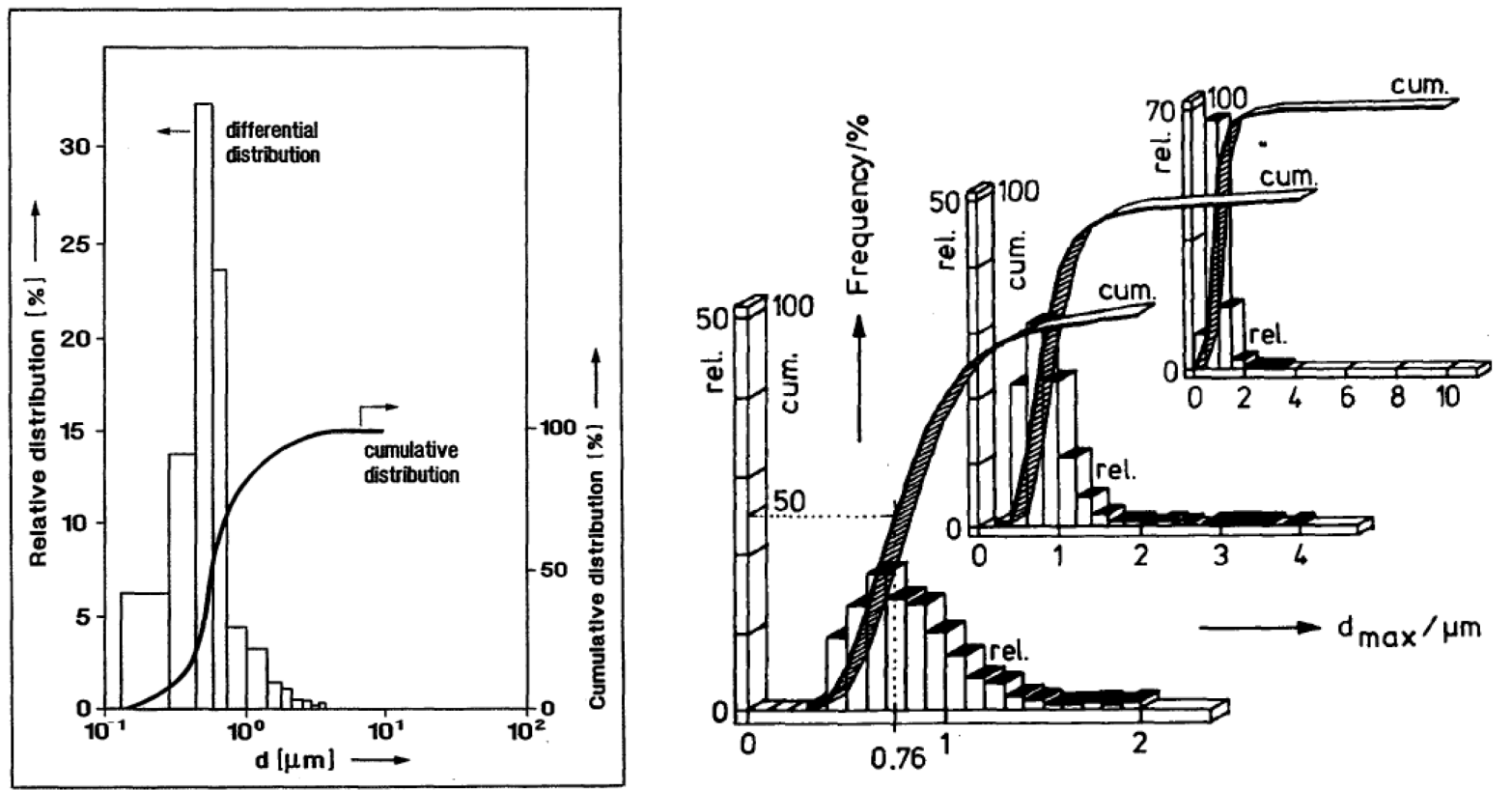

Figure 2. Dust size distributions in AVR (left [IAEA 1997]; right [von der Decken \& Wawrzik 1990]). 
This is possible if $\mathrm{CO}$ and $\mathrm{H}_{2}$ concentrations are high in a reducing gas atmosphere, and in the presence of a suitable catalyst. Such catalysts include iron carbides and metals, but not metal oxides; however, $\mathrm{Fe}_{3} \mathrm{O}_{4}$ can reduce to metallic iron if $\mathrm{H}_{2} / \mathrm{H}_{2} \mathrm{O}$ is $>10$ (high levels of $\mathrm{H}_{2}$ were present in AVR). As chromium oxides (as would be expected in the presence of high nickel alloys) are not a suitable catalyst, and provided that impurity concentrations are controlled, this mechanism should not be present in a future HTGR. Furthermore, this generation mechanism would be active in either a prismatic or pebble bed reactor. The apparent absence of graphite dust in Fort St. Vrain (FSV; Section 2.2.1.3) suggests it was not.

\subsubsection{Thorium Hochtemperatur Reaktor (Germany)}

The German Thorium Hochtemperatur Reaktor (THTR) was a 300 MW(e) pebble bed reactor that operated from 1983 to 1988 and used (Th,U)O $\mathrm{O}_{2}$ BISO fuel. Limited information is available on dust in THTR and its associated activity; in situ measurements of plate-out and activity were precluded by the presence of a concrete reactor vessel, which acted as a biological shield (EPRI 2004). Following 300 fullpower days, dust was collected from moisture sensors in an effort to estimate its quantity. That expected value was $25 \mathrm{~kg}$ with a predicted range of 13 to $400 \mathrm{~kg}$. Elsewhere (Baumer, Kalinowski, Rohler, Schoning, \& Wachholz 1990) it is noted that, while filtration was necessary in THTR to remove dust, filtration was effective, and dust posed no safety concern.

\subsubsection{HTR-10 (China)}

The Chinese HTR-10 is a $10 \mathrm{MW}(\mathrm{t})$ prototype pebble bed reactor. Operating since 2003, there does not appear to be any information available on the nature or quantity of dust in the reactor. Some information obtained from experiments in support of HTR-10 is discussed below.

\subsection{Prismatic Reactors}

\subsubsection{Operational Experience and Legacy Data}

As pebble friction is the primary dust generation mechanism in that reactor type, we can expect significantly less dust to be present in a prismatic reactor. Nevertheless, small quantities of dust may be present in a reactor of this type for a variety of reasons (Hanson 2008):

- Foreign material introduced during construction or refueling

- Friction or erosion of prismatic fuel and reflector surfaces exposed to helium

- Foreign material from interfacing systems

- Corrosion or erosion of metallic surfaces in the coolant system

- CO decomposition.

The nature of these sources seems to preclude any sort of mechanistic modeling of dust generation in a prismatic reactor, and there does not appear to be any ongoing research on the topic. Past experience will have to serve as a basis for dust generation and size information for a future prismatic reactor. Conservative (below) and best estimate recommendations are given by (Hanson 2008) based on Peach Bottom, FSV, and High Temperature Engineering Test Reactor (HTTR) data:

- 0.01 to $10 \mu \mathrm{m}$ graphite particles

- $3 \mathrm{mg} / \mathrm{m}^{3}$ gas concentration

- $5 \mathrm{~g} / \mathrm{m}^{2}$ surface loading. 
Dust is assumed to have no impact on FP transport in the Design Data Needs (DDN)/PIRT reconciliation report for the prismatic reactor discussed below in Appendix A on the basis of Peach Bottom and FSV data. With regard to Peach Bottom, this assumption appears to be based on success in modeling the actual FP distribution with the PAD code (Hanson, Baldwin, \& Selph 1976), which calculates FP transport and sorption on surfaces, without consideration of dust.

If the effect of dust in a prismatic reactor is not important, it should be possible to demonstrate this fact with a representative system code model using the prescribed loading and properties given above. Simple, conservative assumptions regarding the more uncertain physical processes discussed below can be made initially and then refined if the consequences of even relatively little dust are significant.

\subsubsection{Dragon (England)}

Limited information is available on particulate from the Dragon HTGR - a prismatic reactor that operated in England from 1965 to 1976. Particles in Dragon were found to be only 20 to $25 \mathrm{wt} \%$ carbon, the remainder being metallic oxides (Hanson \& Bolin 2007). The (presumably more detailed) reference therein was not available at the time of this writing.

\subsubsection{Peach Bottom}

Peach Bottom Unit 1 was a $115 \mathrm{MW}(\mathrm{t})(40 \mathrm{MWe})$ prototype HTGR that operated for 7 years until 1974. An extensive end-of-life R\&D program was conducted, and a considerable amount of information on dust is available as a result.

Dust in Peach Bottom was sampled in several ways, including plate-out probe and cascade impactor sampling, removal from cyclone filters, and examination of deposits on the steam-generator tubes at the end of life; a detailed report on the sampling and characterization efforts is available (Dyer, Wichner, Martin, \& de Nordwall 1977).

The composition of the Peach Bottom dust was determined by $\mathrm{x}$-ray fluorescence and x-ray diffraction. The former, which was incapable of detecting carbon (or oxygen), revealed that the remaining dust was 50 to $80 \%$ iron, 20 to $50 \%$ silicon, 1 to $5 \%$ sulfur, and the remainder other materials. X-ray diffraction measurements indicated 5 to $25 \%$ graphite dust, and most of the remainder in iron or iron oxides. This technique would also have been unable to detect amorphous carbon.

Subsequently, dust samples were obtained for analysis with a cyclone separator. The composition of these samples was determined by a spark emission spectrographic method, except for carbon, which was measured by burning the sample and measuring the resultant $\mathrm{CO}_{2}$. Its composition was primarily $(\sim 50 \%)$ graphite, with a significant $(\sim 25 \%)$ amount of iron. The material was separated with a sieve, and most of the particulate occupied the smallest $(<44 \mu \mathrm{m})$ or second smallest size bin. The finer particles in two of these samples were also analyzed with a Zeiss analyzer to characterize their size distribution; they range from submicron to $\sim 10 \mu \mathrm{m}$, with a most frequently observed diameter of around $2 \mu \mathrm{m}$ (Figure 3 ).

Samples collected from fuel element surfaces in Peach Bottom contained fine, soot-like material that was presumably created from oil injected during an oil ingress event. There was difficulty in deagglomerating the material to characterize its size, but it was generally submicron (0.1 to $0.4 \mu \mathrm{m})$, and primarily $(>90 \%)$ carbon. If the oil ingress is a significant source of Peach Bottom dust, it may not be characteristic of that to be expected in future gas reactors. 


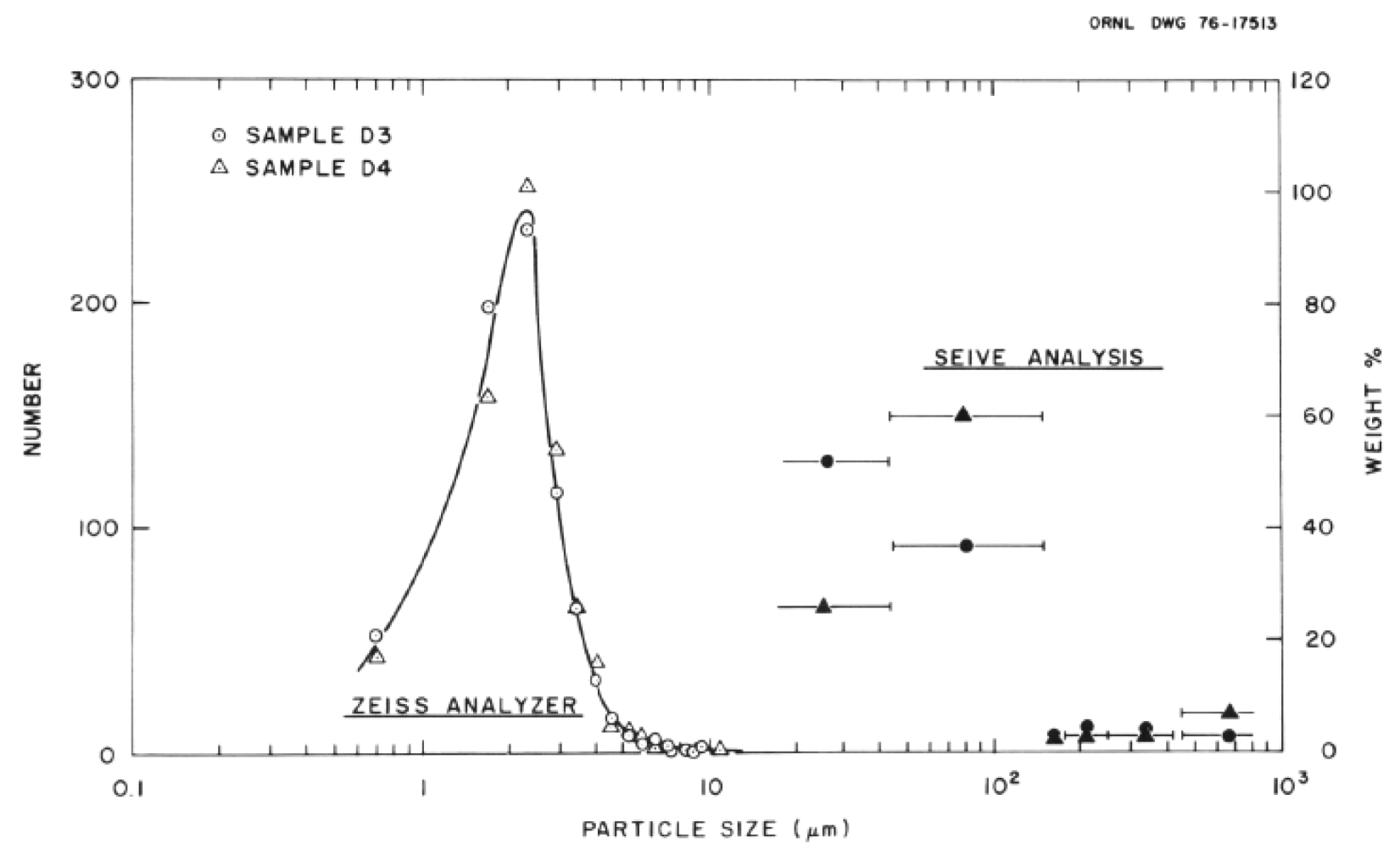

Figure 3. Size distribution of fine Peach Bottom dust (Dyer, Wichner, Martin, \& de Nordwall 1977).

\subsubsection{FSV (Fort St. Vrain)}

FSV has a $842 \mathrm{MW}(\mathrm{t})(330 \mathrm{MW}(\mathrm{e}))$ prismatic reactor that operated from 1974 to 1989 using high enriched (U,Th) $\mathrm{C}_{2}$ TRISO fuel. As with the THTR, FSV had a prestressed concrete reactor vessel (Hanson 2008) that would not have allowed for in situ measurement of dust. What little is known is taken from a $0.7 \mathrm{~g}$ sample of dust obtained from the circulator when it was replaced. The composition of the FSV dust, determined by $\mathrm{x}$-ray diffraction and fluorescence, was primarily $\mathrm{Fe}_{3} 0_{4}(90 \%)$ with the remaining $10 \% \mathrm{MoS}_{2}$. The source of the $\mathrm{MoS}_{2}$ was a lubricant used on the bolts that mounted the circulator, and was also used on the control rod drive cables; $\mathrm{Fe}_{3} \mathrm{O}_{4}$ resulted from chronic water ingress (Sparks et al. 1991). As a prismatic NGNP will not use water bearings, this source of dust appears to not apply to such a design. Interestingly, no graphite was found.

The particulate sampled in FSV was examined by scanning electron microscopy and back-scatter electron microscopy. This is briefly discussed in (Sparks et al. 1991), and only a few general statements are made regarding the size of the dust (no attempt seems to have been made to characterize its distribution). Most particles were agglomerates with diameters less than $1 \mu \mathrm{m}$; a significant number were less than $0.2 \mu \mathrm{m}$.

\subsubsection{HTTR (Japan)}

The Japanese HTTR is a $30 \mathrm{MW}(\mathrm{t})$ prismatic gas-cooled reactor operated by the Japan Atomic Energy Agency (JAEA). To date, limited information is available on dust in the HTTR. ${ }^{\text {a }}$ Thus far, most material collected has been generated by abrasion of graphite piston rings (Hanson 2008), and no contribution from the core is known. The particles are composed of graphite or amorphous carbon, and

a. Some data is available from JAEA, but proprietary. 
have diameters in the range of 1 to $3 \mu \mathrm{m}$; about $1 \mathrm{~kg}$ was collected on primary circuit prefilters. The quantity observed to date is apparently small enough that JAEA has determined full-flow filters for dust will not be necessary. Information on HTTR dust is perhaps more relevant to future designs, as it has not suffered the oil or water ingress that generated significant dust in Peach Bottom and FSV, respectively.

\subsection{Recent and Ongoing Research}

Recent attempts tried to model dust production mechanistically using the PEBBLES code, which tracks the movement of pebbles in a discrete element method. Based on the friction load on each pebble and given a wear coefficient, the dust production rate in the core can be estimated. A model of AVR indicated production of only $4 \mathrm{~g} / \mathrm{y}$ (Cogliati \& Ougouag 2008), far lower than the $3 \mathrm{~kg} / \mathrm{y}$ present. This may be consistent with the hypothesis that most AVR dust was created by abrasion between pebbles and the fuel handling system, which was not modeled in the PEBBLES simulation. Experiments on pebble friction in support of HTR-10, using a pebble force estimate based on the hydrostatic pressure, arrived at a generation rate of $\sim 3 \mathrm{~g} / \mathrm{y}$ (Cogliati, Ougouag, \& Ortensi 2010). ${ }^{\mathrm{b}}$ A PEBBLES model of HTR-10 arrived at a similarly low result of $4.7 \mathrm{~g} / \mathrm{y}$ (Cogliati \& Ougouag 2010). A primary difficulty in the simulations is the wear coefficient, which depends on conditions such as temperature. Little data are available at VHTR relevant conditions, and many experiments are conducted in air at room temperature. There is even conflicting data on the trend of wear coefficient with temperature; different experiments have found it to increase or decrease with increasing temperature (Cogliati, Ougouag, \& Ortensi 2010). In order to achieve an estimate of dust production with PEBBLES that compare to the AVR, the wear coefficient must increase several orders of magnitude from best-estimate values. While wear coefficients of this magnitude have been observed in the so-called "dusting" regime of graphite contact in air above $200^{\circ} \mathrm{C}$, it is not clear that these should also apply in helium.

Experiments on dust generation by pebble friction are being performed at the University of Idaho and the University of Missouri under the auspices of Nuclear Energy University Program and Nuclear Energy Research Initiative, respectively. The Idaho facility is presently under construction, and they are planning to test graphite-graphite and graphite-steel abrasion at VHTR conditions (helium at VHTR temperature and pressure). Some properties of dust produced by friction between graphites have been measured in the University of Missouri (Loyalka 2010) experiment. It uses a drill press to control friction loading between spheres of graphite (grades designed for pebble bed use), sweeps particles out by helium flow, and measures their size distribution as a function of time. The distribution, found to change in time as shown in Figure 4, was presumably a function of wear, and did not have either a normal, lognormal, or exponential distribution. This experiment is conducted at room temperature; the effects of high temperatures are presently unknown.

b. The original reference (in Chinese) is summarized in this document. A presentation on the topic (by S. Yu) is also available on the dust website. 


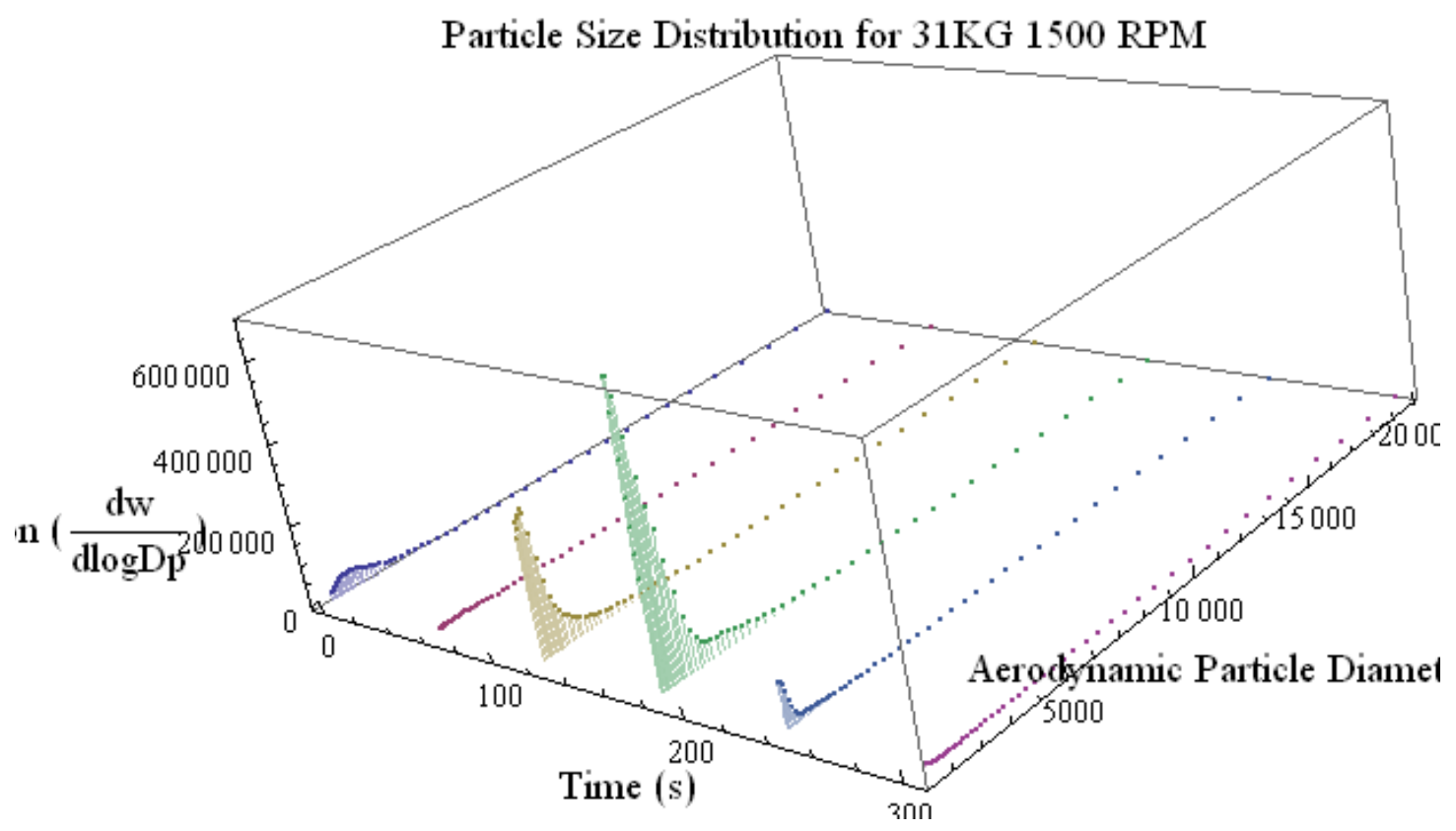

Figure 4. Particle size distribution as a function of time in the University of Missouri experiment.

\subsection{Gap Analysis}

Though the consensus remains that dust is produced primarily by friction in pebble bed reactors, attempts to-date to model dust production with frictional models give estimates far lower than what was observed in AVR. Two apparent shortcomings of the modeling are the failure to include the fuel handling system, which is a significant source of dust production, and uncertainty in wear coefficients.

Both will need to be informed by experiments, and several are ongoing. Key points for future experiments include:

- Based on the graphite under consideration, it is highly desirable that A3-3 and A3-27 matrix material, not just structural graphite, be tested.

- Based on the conditions of the experiment, wear can be expected to vary with the composition, pressure, and temperature of the surround gas. These should be as close to VHTR conditions (helium at high temperature and pressure) as possible.

- In consideration of fuel handling system components, China is presently conducting these experiments.

\subsection{Planned Research}

The research plan for dust production (and all other dust issues) depends strongly on the NGNP design (pebble bed or prismatic) that is ultimately selected.

Experience at Peach Bottom, FSV, and HTTR thus far indicates that little carbonaceous dust can be expected in a prismatic reactor. Oil ingress at Peach Bottom created some, but there should not be a source of oil in an NGNP. In the event the prismatic design is chosen, a research program on dust need not be planned.

Since the quantity of carbonaceous dust for a pebble bed reactor may be large, a research program will be necessary. In order to estimate dust production in a pebble bed reactor, further experimental and 
theoretical work is required. On the experimental side, small-scale friction testing of pebbles is necessary. Friction/tribology studies are already called out in the Graphite Technology Development Plan (Windes 2007) and Methods Technical Program Plan (Gougar 2010). In order to provide relevant data, these experiments must:

- Be performed on the appropriate matrix material (e.g., A3-3)

- Be performed in a helium atmosphere at NGNP temperatures and pressures

- Investigate both friction between pebbles and between pebbles and structural materials (e.g., fuel handling system components).

If they can be made available, irradiated samples should be tested because their wear properties may be altered; friction tests on irradiated graphite are mentioned in the Graphite Technology Development Plan (Windes 2007). Again, these should be conducted on matrix materials such as A3-3.

In addition to the quantity of dust generated, the analysis of data resulting from these experiments should rigorously quantify properties relevant to subsequent transport, including:

- Size distribution

- $\quad$ BET surface area (distribution)

- Mass/density/porosity

- Shape factors.

Further theoretical investigations with PEBBLES should also be carried out. New models should focus on:

- Inclusion of relevant wear coefficients obtained through the experiments outlined above

- Expansion of the models to include the fuel handling system, where significant wear is expected to occur.

In the event that a large discrepancy still exists between data (e.g., from AVR) and models after this work has been carried out, investigation of alternate dust generation mechanisms (such as chemical reactions) may be added to the plan.

This series of dust generation tasks is among the highest priority dust R\&D issues. 


\section{DUST DEPOSITION}

Dust particles will deposit on surfaces under the influence of various forces. Potentially important deposition mechanisms include ${ }^{c}$ :

- Gravitational

- Diffusive

- Thermophoretic

- Turbulent

- Inertial

- Electrostatic.

\subsection{Operational Experience}

There are established (well verified and validated) models for deposition by gravity, diffusion, thermophoresis, turbulence, and inertia; a summary of the relevant equations and comprehensive list of more detailed references is available (NEA 2009). The models for deposition by gravity, diffusion, and thermophoresis are included in MELCOR (MELCOR 2005), and the turbulent and inertial deposition models added to a modified version for fusion (Merrill 2010) will be incorporated into subsequent versions of MELCOR. Thus, these phenomena should not require further study.

Electrostatic deposition (or, more generically, electrostatic transport) is another matter. While the potential importance of electrostatic effects is recognized for even LWRs, they appear to have received comparatively little study, and there appears to be no consensus as to the importance of these effects (NEA 2009). Electrostatic models are absent from system codes such as MELCOR, and would be difficult to implement.

\subsection{Recent and Ongoing Research}

Aerosols are electrostatically charged because of the radiation environment (NEA 2009; NRC 2008). The radiation can form ions in the gas phase that can charge dust particles when they collide. Higher mobility of negative ions is expected to lead to negatively charged aerosol particles. Electrostatic repulsion of particles in the gas could then inhibit the usual agglomeration that accelerates deposition processes. Charged particles deposited on a surface may similarly repel particles that approach and would otherwise be deposited there.

An apparently competing effect of electrophoresis is also described (NEA 2009), where particles are stated to carry a positive charge due to $\beta^{-}$and $\alpha$ decay (which strips away more electrons that the positive charge it carries) occurring within the particle. Small particle sizes and high concentrations would serve to limit the amount of self-charging possible.

Equations describing the (positive and negative) ion distribution and aerosol charge have been presented (Clement \& Harrison 2000). Solutions of the aerosol charge distribution have been obtained by a direct simulation monte carlo method (Loyalka 2010) incorporating electrostatic agglomeration and diffusion for a given initial charge distribution. It is not clear how the initial condition has been prescribed; it is presumably determined by the radiation environment and the FP content of the particles, which are likely highly uncertain or unknown.

c. Diffusiophoresis may also be important, but this mechanism is omitted from the present list because it is related to water condensation and evaporation. 


\subsection{Gap Analysis}

While most transport and deposition phenomena are well understood, electrostatic effects are an exception; the present state of knowledge is limited. Continuing fundamental studies are needed to:

- Determine the radiation field and FP content of particles that would give rise to electrostatic forces comparable to other particle forces.

- Provide input on how to proceed with a system model in the event these conditions are likely or achievable.

\subsection{Planned Research}

As the fundamental mechanisms of dust deposition are well understood, it is largely unnecessary to incorporate new research on these subjects into NGNP Project plans. Electrostatic transport and deposition is a possible exception. However, since modeling of nuclear aerosols has not historically been noticeably hindered by the lack of electrostatic transport models, and adequate tools for modeling concurrent thermal-hydraulic and electrostatic aerosol transport do not exist, no new research on this topic is planned. Fundamental studies (e.g., under the auspices of NEUP) should continue to be supported. 


\section{DUST DISTRIBUTION UNDER NORMAL OPERATION}

If dust is continuously being generated, its distribution will be changing in time over the life of the plant. The consequences that result from an accident will then depend on when the accident occurs in this life time and what the dust distribution is at that time the initial condition for the accident calculation forms. Dust generation and the evolution of particle size distribution thus need to be modeled over long time periods.

\subsection{Operational Experience and Legacy Data}

While it is impossible to know precisely what the dust distribution was in any operating reactor, AVR experience indicates that most dust is, in fact, deposited, and circulating concentrations are very low. The cold gas filter experiment consisted of a bypass flow of $8 \mathrm{~m}^{3} /$ hour through a quartz paper filter, which captured dust larger than $3 \mu \mathrm{m}$ at an efficiency of $50 \%$. Forty-five experiments were conducted over many years of operation, allowing circulating dust concentrations to be determined as shown in Figure 5. Concentrations during steady-state were 1 to $2 \mu \mathrm{g} / \mathrm{m}^{3}$, increasing to an average over the final operating years of $5 \mu \mathrm{g} / \mathrm{m}^{3}$. It is apparent from Figure 5, that at times, concentrations could be considerably higher; but because the total estimated dust production is $3 \mathrm{~kg} / \mathrm{y}$ resulting in $60 \mathrm{~kg}$ at the end of life, and $5 \mu \mathrm{g} / \mathrm{m}^{3}$ corresponds to only $8 \mathrm{mg}$, the overwhelming majority of dust remained deposited on surfaces. Minor disturbances temporarily raised the circulating dust quantity. The lines marked T1, T2, and T3 are transients. It is estimated that less than $0.1 \%$ of deposited dust was mobilized in these situations, and that 0.5 to $2 \%$ of circulating dust was redeposited on each circulation.

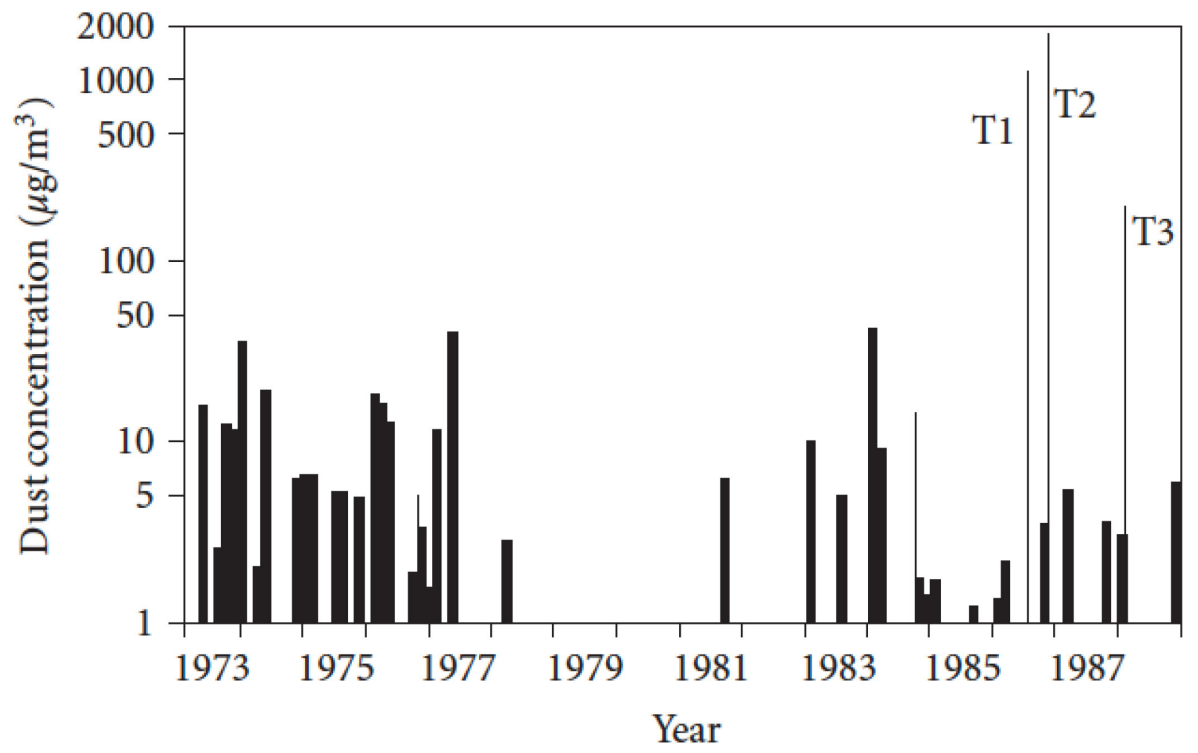

Figure 5. Circulating dust concentrations in AVR (Moorman 2008).

\subsection{Recent and Ongoing Research}

Future HTGR safety analysis will certainly employ system codes in accident analyses. Establishing the initial condition for such accidents presents a difficulty for these codes: dust generation and transport must be modeled over very long periods of time. There is, however, an established method of dealing with the problem in systems codes, and it is employed in both SPECTRA (Westinghouse 2008) and MELCOR (MELCOR 2005). Time steps in thermal hydraulic calculations are limited by the courant limit, but it is assumed that dust is influenced by, but does not in turn influence, these conditions. A 
thermal hydraulic steady-state can then be established at which point those flow conditions are fixed and dust transport is calculated with a much longer time step. This allows for dust transport calculations over an entire plant lifetime.

An analysis of this type has been performed for the 500 MW Pebble Bed Modular Reactor (PBMR) demonstration power plant using SPECTRA. In the analysis, a dust generation rate of $28 \mathrm{~kg} / \mathrm{fpy}$ is extrapolated from AVR data. This results in a total of 1,632 $\mathrm{kg}$ of dust present after 57 years of full power operation. Most $(1,420 \mathrm{~kg})$ of the dust generated is deposited in the reactor vessel, with smaller amounts (10 to $130 \mathrm{~kg}$ ) residing in the intermediate heat exchangers, core inlet and outlet pipes, and helium purification system filter.

\subsection{Gap Analysis}

Analyses like those described above predict the integral effect that results from the combination of separate effects described in the previous section. However, certain integral effects may not be captured by such a method. For example, there is a possibility that dust may be rather inhomogeneously distributed, with accumulation primarily occurring in "dead zones" of low flow. Other aspects of the design, such as pipe bends or entrance effects, may also influence the distribution of dust under normal operation. The ability of existing tools to treat these phenomena needs to be assessed. Integral experiments such as the THAI facility (Section A-2 in Appendix A) could be able to provide this sort of data.

\subsection{Planned Research}

The dust distribution under normal operation is essentially governed by the well-known fundamental phenomena described in Section 3, and system codes such as MELCOR or SPECTRA are designed to apply these in concert to predict integral results in large facilities. The Methods Technical Program Plan (Gougar 2010) should include a dust (and fission product) transport system modeling task, provided the pebble bed reactor design is chosen. Models for both normal operation at steady-state and relevant accident transients should be included in the task. A similar effort was undertaken with SPECTRA, with which a model of the PBMR was developed (Stempniewicz 2010). Such a model would predict the dust distribution under normal operation and, provided it is built with a suitably fast system code, be used to perform sensitivity studies on the more uncertain transport properties.

The complex geometries of large-scale facilities can give rise to unexpected integral transport results. For this reason, integral data for benchmarking dust transport system codes is valuable. Integral data should be obtained for the purposes of benchmarking from the THAI facility (Section A-2, already included in the Methods Technical Program Plan) or the planned integral facilities of the DUSTIN project (Lind 2010). 


\section{DUSTIAEROSOL GROWTH}

\subsection{Operating Experience and Legacy Data}

Though "aerosol" here may also refer to FP aerosols, dust particles will also agglomerate, and their size distribution may evolve over time. Historically, aerosol transport models were developed for wet conditions such as the steam atmosphere that would result from an LWR accident. Growth may proceed differently in the dry, inert-gas environment of a gas-cooled reactor. In particular, water condensation in agglomerate interstices tends to produce more spherical particles (NRC 2008; NEA 2009), and the absence of this phenomenon in most gas reactor accident scenarios may result in agglomeration of aerosols with very high shape factors. The evolution of shape factors as such dry aerosols agglomerate is not known, and they may additionally depend on the particle size, a factor that codes such as MELCOR are presently not equipped to handle. However, models for Brownian, gravitational, and turbulent (shear and inertial) agglomeration do account for shape factors; these are described in (NEA 2009) and are present in MELCOR (MELCOR 2005). The models incorporate both an agglomeration shape factor $(\gamma)$ and a dynamic shape factor $(\chi)$, which are user inputs.

\subsection{Recent and Ongoing Research}

The agglomeration shape factor $(\gamma)$ and a dynamic shape factor $(\chi)$ are essentially corrections that give the real collision cross section and drag force for an irregular particle, referenced to a diameter of a spherical particle of equivalent volume. Some analytical models are available for shape factors as described in NEA (2009). Models for both shape factors depend on their fractal dimension. Two regimes are described: for very small constituent particles $(0.025 \mu \mathrm{m}$ in this case), they initially formed chains for which the dynamics shape factor increased rapidly with agglomerate size. Eventually these chains folded in on themselves, at which point the shape factor was reduced as illustrated in Figure 6.

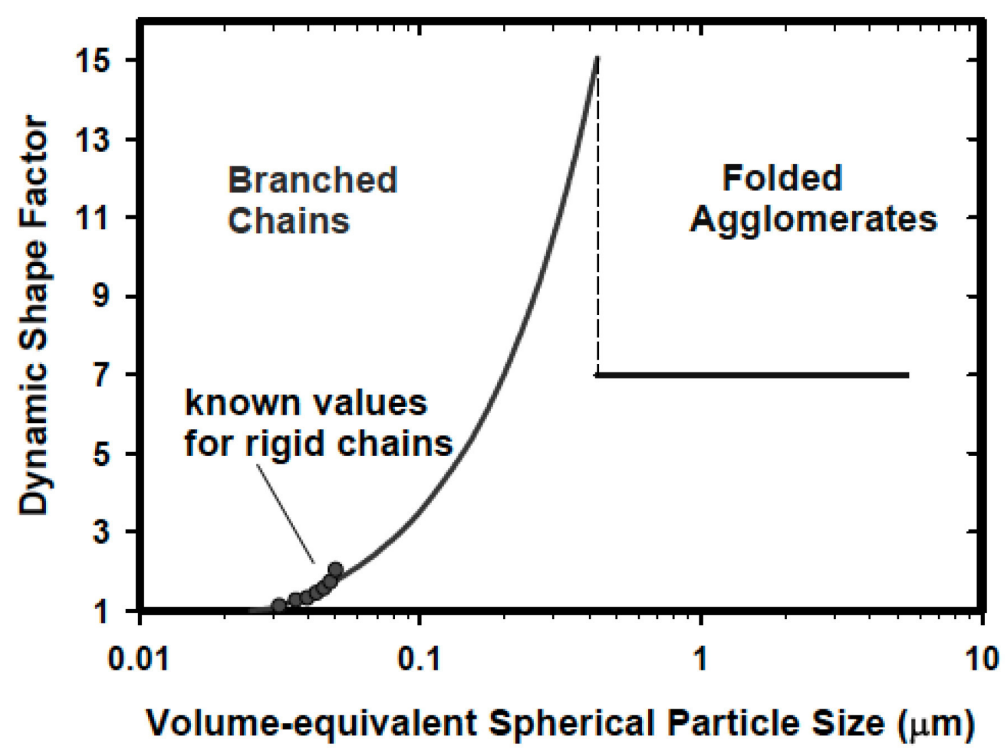

Figure 6. Dynamic shape factor as a function of particles size, from (NEA 2009).

It is not clear if dust particles will be present at such small diameters, so the prior regime may (or may not) be important here. At any rate, it appears that no information related to the shape or fractal dimension 
of characteristic graphite dust (abrasion-generated) is known (e.g., from AVR). Future dust characterization efforts should seek to quantify these variables.

\subsection{Gap Analysis}

As experiments to generate dust from friction are ongoing, these may be a source of useful information on shape factors. This is also a topic that is well suited to sensitivity studies. MELCOR, for example, includes both agglomeration $(\gamma)$ and dynamic $(\chi)$ shape factors in its transport models; these may be altered by changing a single sensitivity coefficient in an input file, and as such should be straightforward to carry out. Studies of this type performed in the past (Reference 8-2 of (NEA 2009), in German) have indicated a large dependence on shape factor, but a similar study on a representative VHTR design is warranted. In the event radionuclide release is highly dependent on shape factors, a more mechanistic determination of them may be necessary. While these are often determined by "fitting" or back calculating (MELCOR 2005), this practice is not recommended (NEA 2009).

\subsection{Planned Research}

Provided it is developed with a suitable code (such as MELCOR) that has the ability to specify shape factors for aerosols, the system modeling effort proposed in Section 4.4 should include sensitivity studies to examine the effect of changing agglomeration and dynamic shape factors on integral dust and fission product transport.

It was proposed in Section 2.5 that properties (including shape factors) of particles generated in friction experiments should be measured. To the extent that these data are available, they should be used to inform the system code analysis. 


\section{DUST BOUNCE AND BREAKUP DURING DEPOSITION}

In the preceding discussion on dust deposition mechanisms, it was implicitly assumed that all particles encountering a wall are deposited. In reality, this is not necessarily the case because particles may bounce on impact and agglomerates may break into their smaller constituents. As growth of particles tends to increase their retention (and thus mitigate radioactive releases) because of increased settling, and decrease the risk of explosion (see Section 9), breakup might be undesirable. This phenomenon does not appear to have received extensive study, and no models for it are available in systems codes such as MELCOR. As with resuspension (see Section 8), the extent to which breakup may occur can be expected to depend strongly on the forces that bind agglomerates, and thus on their surface morphology and contact geometry, which is rather difficult to determine in practice.

\subsection{Operational Experience}

There does not appear to be any information available on these effects from operating experience. Research on this issue has only been conducted more recently.

\subsection{Recent and Ongoing Research}

Theoretical models for particle bounce are based on factors like the coefficient of restitution, which are difficult or impossible to characterize for nuclear aerosols with varying composition and structure. Another approach is correlate bounce probability with particle kinetic energy, as shown in Figure 7 for fly ash.

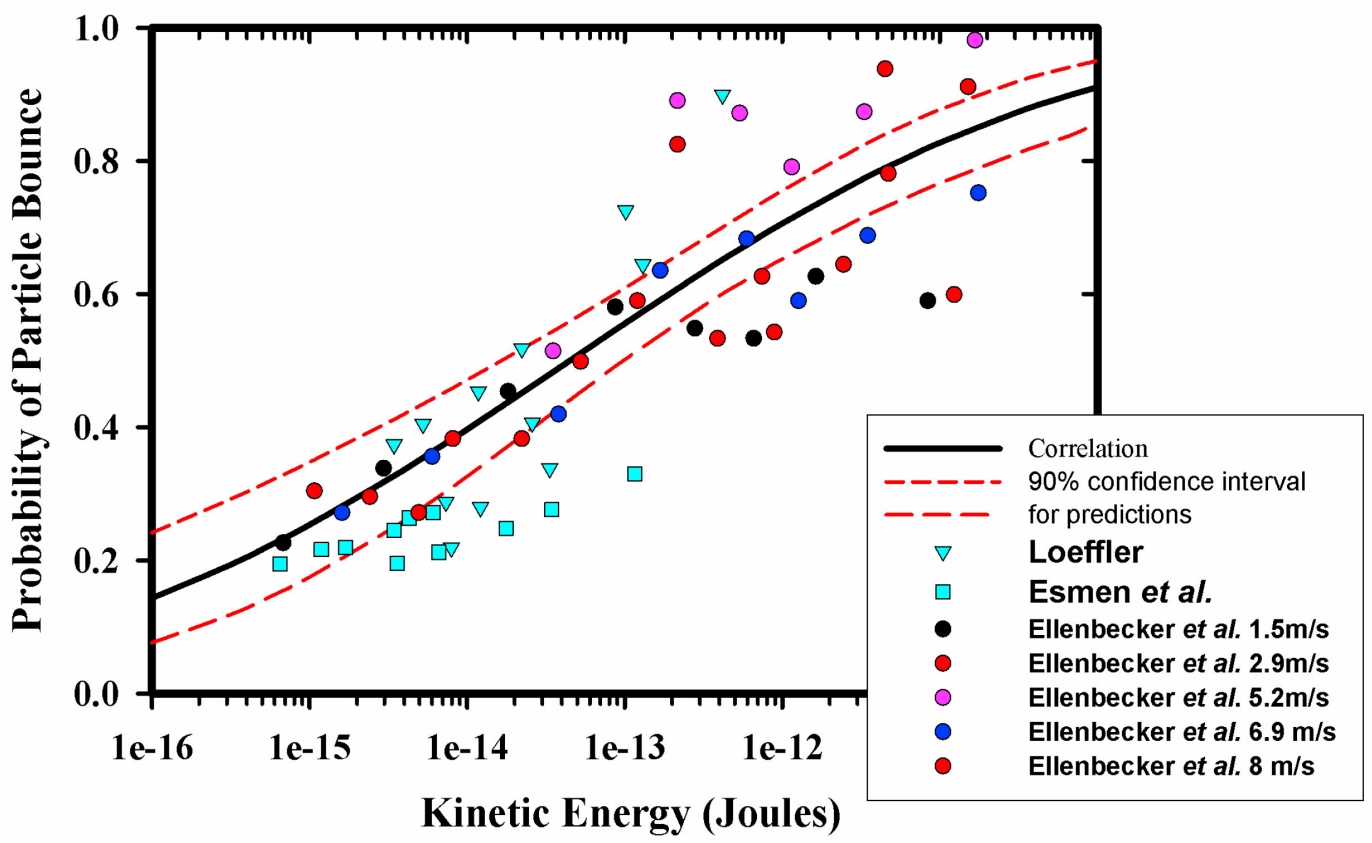

Figure 7. Probability of fly ash particle bounce as a function of kinetic energy (D. Powers, Dust Issues Assessment Meeting). 
Since dust agglomerates in an HTGR are likely to be more fragile, there is a possibility that they will not only bounce, but break, on impact. Phenomena may result in breakup of aerosol particles are:

- Impact with walls

- Shear forces in highly turbulent flows

- Shock waves at pipe exits.

A particular difficulty with wall impact is that particles are presumed to contact surfaces at high rates of speed because of their inertia, but the standard continuum description of aerosols does not include inertial terms for the particles. Inertial deposition in pipe bends is modeled in this case via empirical functions (as described in Section 4.5), but no breakup is accounted for in this model.

Two breakup mechanisms have recently received some experimental and theoretical consideration (Ammar, Lind, Reeks, \& Dehbi 2007). Among the important points in that work were:

- Breakup occurs on shorter timescales than agglomeration

- Turbulence (at $\mathrm{Re}=10^{5}-10^{6}$ ) was dominant over shock-interaction in inducing breakup

- Particle counts indicated breakage of agglomerates into eight constituent particles

- The diameter at breakup was a power law function of the turbulence dissipation rate

- Prior models for the breakage rate indicate dependence on the turbulence dissipation rate and a threshold dissipation rate, but better models are needed that incorporate resuspension.

\subsection{Gap Analysis}

A number of difficulties are apparent if the objective is implementing such a model in system codes. The turbulent dissipation rate was determined here by the use of a computational fluid dynamics (CFD) code; turbulence models of this nature are not present in system codes. In MELCOR, turbulent agglomeration models also depend on the turbulence dissipation rate, but this quantity is simply a user input, and no model is present to calculate it based on flow conditions. In principle, a model of this type could be added to MELCOR and would use that input. However, it is also necessary to know the number of particles into which agglomerates break; this is given as a function of the fractal dimension of the agglomerate (NEA 2009), which, in principle, can be measured but may not be well known. A value of 1.78 is recommended in (NEA 2009), though the applicability of this to graphite aerosols in a gas reactor is not clear.

It is also apparent that an appropriate equation for the evolution of the size distribution must account not only for breakup, but also simultaneous resuspension, deposition, agglomeration, etc.

It will be necessary to use discrete element models and CFD codes to try to assess the importance of breakup. Such models should seek to assess the potential for breakup in conditions that might be encountered in an accident scenario.

\subsection{Planned Research}

The possibility of dust bounce (or breakup) is presently not accounted for in system level codes; the deposition models discussed in Section 3 imply a 100\% sticking probability. Introduction of a simple bounce probability relationship such as that shown in Figure 7, which will be carried out following development of a pebble bed system model as outlined in Section 4.4, should be relatively straightforward. 
Breakup models dependent on either of the turbulence model parameters discussed above, or discrete element models as demonstrated at the Dust Issues Assessment meeting, are more difficult to implement in a system code. Since breakup essentially acts to decrease the particle size distribution, it is recommended that sensitivity studies be performed on the particle size distribution, as with the shape factors described in Section 5.4. Unless demonstrated by these studies, the effect is assumed to be minor and more detailed modeling efforts (e.g., CFD) or experiments on breakup are not proposed at this time. 


\section{DUST/FP INTERACTION}

\subsection{Operational Experience and Legacy Data}

Data on FP interaction with dust from operating experience comes in the form of activity measurements of sampled dust. Available data is summarized in this section by facility.

\subsubsection{Peach Bottom}

Plate-out activities were measured in the diffusion tube experiments at Peach Bottom. Not surprisingly, attempts to quantify the effect of dust were complicated by the fact that the particles were not monodispersed, and large temperature gradients may have affected dust transport. As in other investigations, deposition profiles that were unexpectedly flat were presumed to be a result of the presence of dust.

The activity of dust samples from the cyclone separator was found to be a function of particle size. Most of these sample particles, which were taken over the course of 3 to 4 years and separated by a sieve, were in the smallest $(<44 \mu \mathrm{m})$ size bin. Specific activities for two of these samples are shown in Table 3 to vary widely.

Activities of some isotopes adhered to the soot-like dust on fuel elements were also measured. Specific activity of this dust is reported in Table 4.

Table 3. Specific activity of Peach Bottom Dust as a function of particle size Bottom (Dyer, Wichner, Martin, \& de Nordwall 1977).

Table 5.2-2. Dust sample D2: Particle size distribution by sieve analysis and gamma emitting radionuclide concentrations $(\mu \mathrm{Ci} / \mathrm{g}$, April 24, 1971).

\begin{tabular}{|c|c|c|c|c|c|c|c|c|c|c|c|c|c|c|c|}
\hline $\begin{array}{c}\text { Size } \\
\text { fraction } \\
\text { (um) }\end{array}$ & wto & ${ }^{51} \mathrm{Cr}$ & ${ }^{54} \mathrm{Mn}$ & $59 \mathrm{Te}$ & ${ }^{60} \mathrm{Co}$ & ${ }^{65} \mathrm{zn}$ & ${ }^{90} \mathrm{Sr}$ & ${ }^{95} \mathrm{Zr}$ & $110_{\mathrm{Ag}}$ & ${ }^{106} \mathrm{Ru}$ & ${ }^{125} \mathrm{Sb}$ & ${ }^{134} \mathrm{Cs}$ & ${ }^{137} \mathrm{Cs}$ & ${ }^{144} \mathrm{Ce}$ & ${ }^{154}$ Eu \\
\hline Composite & & $\mathrm{ND}^{\mathrm{a}}$ & 1.3 & 8.5 & 9.5 & 0.5 & 72 & 3.0 & 2.3 & 11.0 & 0.99 & 17.0 & 44.0 & 88.0 & 0.54 \\
\hline$>420$ & 0.86 & 320 & 0.68 & 11.0 & 28.0 & $\mathbb{N D}$ & $\mathrm{b}$ & IND & 0.63 & 2.3 & $\mathrm{ND}$ & 1.5 & 3.6 & 4.2 & $\mathrm{ND}$ \\
\hline $250-420$ & 1.9 & $\mathrm{KD}$ & $\mathrm{WD}$ & $\mathrm{ND}$ & 16.0 & ND & & 180.0 & WD & 356.0 & 34.0 & 480.0 & 1000.0 & 3200.0 & 13.0 \\
\hline $177-250$ & 3.0 & $\mathrm{ND}$ & WD & $\mathrm{ND}$ & 18.0 & ND & & 17.0 & $\mathrm{ID}$ & $36 \mathrm{c} .0$ & 31.0 & 400.0 & 920.0 & 2700.0 & 12.0 \\
\hline $149-177$ & 1.8 & IDD & WD & IND & 17.0 & $\mathbb{N D}$ & & 69.0 & IND & 195.0 & 17.0 & 250.0 & 610.0 & 1700.0 & 11.0 \\
\hline $44-149$ & 33.0 & $\mathrm{ID}$ & WD & & 42.0 & & & 14.0 & 6.33 & 27.0 & 2.6 & 35.0 & 88.0 & 220.0 & 1.0 \\
\hline$<44$ & 60.0 & $\mathrm{ND}$ & 2.8 & 11.0 & 20.0 & 1.1 & & 10.0 & 6.4 & 10.0 & 0.8 & 21.0 & 56.0 & 97.0 & 0.4 \\
\hline
\end{tabular}

Table 5.2-4. Dust sample D4: Particle size distribution and radionuclide concentrations ( $\mu$ Ci/g, October 31, 1974).

\begin{tabular}{|c|c|c|c|c|c|c|c|c|}
\hline $\begin{array}{l}\text { Size } \\
\text { fraction }^{\mathrm{a}} \\
(\mu \mathrm{m}) \\
\end{array}$ & $\mathrm{wt} \%$ & ${ }^{54} \mathrm{Mn}$ & ${ }^{60} \mathrm{Co}$ & $90_{\mathrm{Sr}}$ & $110 \mathrm{~m}_{\mathrm{Ag}}$ & ${ }^{134} \mathrm{Cs}$ & ${ }^{137} \mathrm{Cs}$ & ${ }^{144} \mathrm{Ce}$ \\
\hline Composite & $b$ & 4.0 & 460 & 29 & 4.2 & 99 & 120 & 1.6 \\
\hline$>450$ & $7 \cdot 2$ & 5.4 & 310 & 0.16 & $\leq 2.0$ & 12 & 9 & $\leq 2$ \\
\hline $250-420$ & 3.0 & 5.6 & 275 & 94 & $\leq 2.0$ & 40 & 90 & 200 \\
\hline $177-250$ & 3.6 & 7.8 & 400 & 79 & $\leq 3.0$ & 45 & 174 & 50 \\
\hline $149-177$ & 2.6 & 4.3 & 1000 & 44 & $\leq 5.0$ & 60 & 175 & 86 \\
\hline $44-149$ & 60.0 & 2.3 & 390 & 26 & 3.42 & 101 & 112 & 10 \\
\hline$<44$ & 23.6 & $3 \cdot 3$ & 140 & 28 & 4.89 & 151 & 156 & 6 \\
\hline
\end{tabular}


Table 4. Specific activity of soot-like dust in Peach Bottom (Dyer, Wichner, Martin, \& de Nordwall 1977).

\begin{tabular}{|c|c|c|}
\hline & \multicolumn{2}{|c|}{ Soot deposit removed from fuel element } \\
\hline & 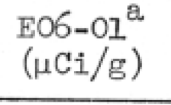 & $\begin{array}{l}\mathrm{FO} 05-05^{\mathrm{b}} \\
(\mu \mathrm{Ci} / \mathrm{g})\end{array}$ \\
\hline $\mathrm{Mn}-54$ & 29.4 & 32.5 \\
\hline Co-60 & 53.1 & 84.9 \\
\hline $\mathrm{Zr}-95$ & 2.2 E3 & \\
\hline $\mathrm{Nb}-95$ & $2.0 \mathrm{E} 5$ & \\
\hline $\begin{array}{l}\text { Ag-110m } \\
\text { Sb-125 }\end{array}$ & 4.84 & 34.0 \\
\hline Cs -134 & 29.1 & $4.19 \mathrm{E} 3$ \\
\hline Cs -137 & 87.8 & $2.38 \mathrm{E} 3$ \\
\hline $\mathrm{Ce}-144$ & 1.03 E3 & \\
\hline $\begin{array}{l}\mathrm{Eu}-154 \\
\mathrm{H}-3\end{array}$ & & 36.7 \\
\hline $\mathrm{Sr}-89$ & $1.0 \mathrm{E} 3$ & \\
\hline $\mathrm{Sr}-90$ & 25.0 & \\
\hline
\end{tabular}

\subsubsection{FSV}

Some information on dust and associated activity is available from a plate-out probe experiment at FSV (Burnette 1982). Deposition of vapor phase FPs on the surface is not distinguished from deposition of dust particles containing FPs in this experiment. But, as deposition profiles in the absence of dust are expected to be steep, more uniform deposition profiles may suggest the involvement of dust. Sr-90 and Cs-137 measurements seemed to suggest this, but the relative absence of similar activity on the dust filters could not initially be explained. The presence of Cs-134, however, suggests significant Cs-dust interaction. Cs-134 is an activation product of Cs-133. As gaseous Cs-134 was expected to plateout on cold surfaces long before reaching the probe and neutron activation could not have occurred near the probe, it was concluded that Cs-134 was present because of the attachment of Cs-133 to dust, which became activated on passing through the core, and was transported all the way to the probe. As Cs-134 activity was also largely absent on the dust filter, it was apparent that dust particles must have been small enough to pass through it. The conclusions were that Cs-137 and Sr-90 were totally associated with dust, that significant fractions of Ba-140 and Sr-89 were similarly associated with dust, but that iodine was not associated with dust. Though activities were measured in the experiment, nothing appears to be known about the quantity or size of dust from this experiment, so an activity per mass of dust cannot be inferred.

\subsubsection{AVR}

The cold gas filter experiment consisted of a bypass flow of $8 \mathrm{~m}^{3} /$ hour through a quartz paper filter, which captured dust larger than $3 \mu \mathrm{m}$ at an efficiency of 50\%. Forty-five experiments were conducted over many years of operation, which allowed for a determination of circulating dust concentrations over those years as shown in Figure 5. Concentrations during steady-state were 1 to $2 \mu \mathrm{m} / \mathrm{m}^{3}$, increasing to an average over the final operating years of $5 \mu \mathrm{m} / \mathrm{m}^{3}$. It is apparent from Figure 5, that at times, concentrations could be considerably higher; but as the total estimated dust production is $3 \mathrm{~kg} / \mathrm{y}$ resulting in $60 \mathrm{~kg}$ at the end of life, and $5 \mu \mathrm{m} / \mathrm{m}^{3}$ corresponds to only $8 \mathrm{mg}$, most dust was deposited on surfaces. Minor disturbances temporarily raised the circulating dust quantity. The lines marked T1, T2, and T3 are transients. It is estimated that less than $0.1 \%$ of deposited dust was mobilized in these situations, and that 0.5 to $2 \%$ of circulating dust was redeposited on each circulation. 


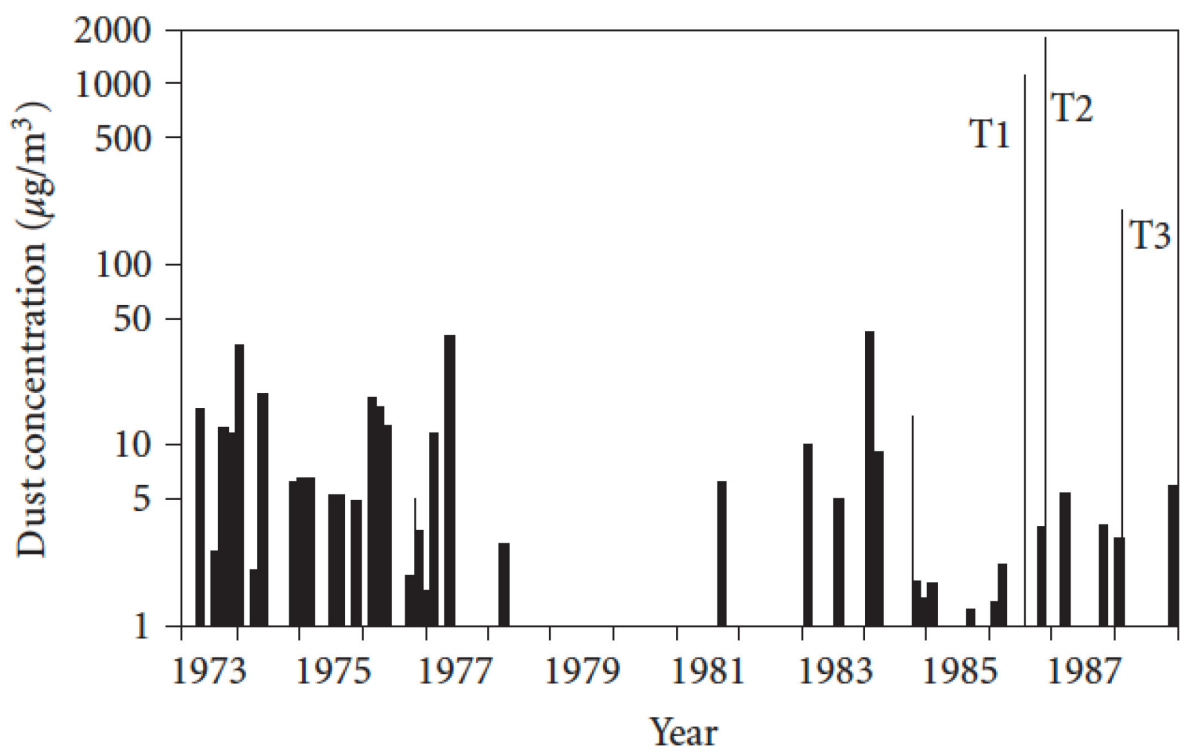

Figure 8. Circulating dust concentrations in AVR (Moorman 2008).

The primary objective of the dust experiment was to measure FP activity of the vapor phase and that associated with dust. Results of several experiments are summarized in Table 5. The experiments were run for varying lengths of time; there does not appear to be any clear correlation between run time and collected dust mass. Similarly, the specific activity of the dust does not appear to be correlated strongly with the coolant activity. For Ag-110m in particular, the coolant activity appears to be relatively constant through most of the tests, less so for Ag-110m on dust, which varied between $5.4 \times 10^{4} \mathrm{~Bq} / \mathrm{g}$ and $5.43 \times 10^{7} \mathrm{~Bq} / \mathrm{g}$. Cs-137 dust activity was between $1.13 \times 10^{6}$ and $9.82 \times 10^{8} \mathrm{~Bq} / \mathrm{g}$. Elsewhere, it is stated that cesium and strontium deposition were 2 to 2.5 times higher in Branch 2 of the experiment because of the presence of a dust filter there. This appears to be generally true of cesium and silver activity in Table 5, though it is not clear why dust in this location would have an activity significantly different than dust extracted from other locations.

Table 5. Results of AVR dust experiments (IAEA 1997).

\begin{tabular}{|c|c|c|c|c|c|c|c|c|}
\hline \multirow{2}{*}{$\operatorname{Run}^{(1)}$} & \multirow{2}{*}{$\begin{array}{c}\text { Time } \\
{[\mathrm{h}]}\end{array}$} & \multirow{2}{*}{$\begin{array}{c}\text { Total coolant } \\
\text { throughput in } \\
\text { branch } 2\left[\mathrm{Nm}^{3}\right]\end{array}$} & \multirow{2}{*}{$\begin{array}{c}\text { Average } \\
\text { gas tempe- } \\
\text { rature } \\
{\left[{ }^{\circ} \mathbf{C}\right]}\end{array}$} & \multirow{2}{*}{$\begin{array}{c}\text { Dust collected } \\
\text { on SFs [mg] } \\
\text { branch } 1 / \\
\text { branch } 2\end{array}$} & \multicolumn{2}{|c|}{$\begin{array}{c}\text { Specific dust activity } \\
{\left[10^{6} \mathrm{~Bq} / \mathrm{g}\right]}\end{array}$} & \multicolumn{2}{|c|}{$\begin{array}{c}\text { Coolant activity } \\
{\left[\mathrm{Bq} / \mathrm{Nm}^{3}\right]}\end{array}$} \\
\hline & & & & & Cs-137 & $\mathrm{Ag}-110 \mathrm{~m}$ & Cs-137 & Ag-110m \\
\hline$S_{1}$ & 146 & 5,287 & 846 & $81.9 / 119.4$ & $1.13 / 1.64$ & $0.057 / 0.054$ & $17.8 / 37.0$ & $0.89 / 1.22$ \\
\hline $\mathrm{S}_{2}$ & 764 & 27,820 & 850 & $42.6 / 46.6$ & $1.27 / 9.45$ & $0.11 / 0.32$ & $1.85 / 15.9$ & $0.17 / 0.52$ \\
\hline$s_{3}$ & 432 & 15,539 & 850 & $1.1 / 4.3$ & $31.1 / 41.9$ & $3.16 / 2.06$ & $2.22 / 11.5$ & $0.22 / 0.59$ \\
\hline$S_{4}$ & 288 & 10,452 & 850 & $3.5 / 3.0$ & $6.94 / 29.4$ & $0.78 / 1.57$ & $2.22 / 8.51$ & $0.26 / 0.44$ \\
\hline$S_{5}$ & 430 & 15,451 & 950 & $7.4 / 6.7$ & $13.3 / 96.1$ & $1.24 / 4.39$ & $6.29 / 41.8$ & $0.59 / 1.92$ \\
\hline$S_{6}$ & 431 & 15,222 & 950 & $5.3 / 12.0$ & $16.7 / 50.3$ & $1.88 / 2.90$ & $5.92 / 39.6$ & $0.67 / 2.29$ \\
\hline$S_{7}$ & 432 & 15,314 & 950 & $4.8 / 8.6$ & $15.2 / 61.5$ & $1.84 / 3.76$ & $4.81 / 34.4$ & $0.59 / 2.11$ \\
\hline$S_{9}$ & 288 & 9,975 & 950 & $5.4 / 4.2$ & $2.48 / 25.0$ & $0.64 / 2.37$ & $1.48 / 10.4$ & $0.33 / 1.00$ \\
\hline$S_{10}$ & 744 & 26,062 & 950 & $2.5 / 8.4$ & $13.1 / 28.3$ & $4.31 / 4.08$ & $1.48 / 9.25$ & $0.41 / 1.33$ \\
\hline$S_{11}$ & 188 & 6,604 & 900 & $5.9 / 7.5$ & $31.2 / 98.2$ & $15.3 / 54.3$ & $27.8 / 112$. & $13.7 / 61.7$ \\
\hline $\mathrm{S}_{12}$ & 680 & 24,015 & 950 & $4.3 / 17.2$ & $28.3 / 29.0$ & $11.0 / 7.46$ & $5.18 / 20.7$ & $1.96 / 5.33$ \\
\hline $\mathrm{S}_{13}$ & 668 & 24,434 & 950 & & & & & \\
\hline $\mathrm{S}_{14}$ & 722 & 26,138 & 935 & & & & & \\
\hline$S_{15}$ & 275 & 10,155 & 933 & & & & & \\
\hline
\end{tabular}


Specific activities of AVR dust taken from different locations in the primary circuit are provided in Table 6, and vary over several orders of magnitude for a given isotope. Though iodine was previously assumed not to interact with dust, activities of $3.5 \times 10^{6} \mathrm{~Bq} / \mathrm{g}$ were found. Based on the estimated inventory of iodine of $25 \times 10^{9} \mathrm{~Bq}$, this was taken as evidence that iodine was not evenly distributed amongst the total mass of dust, and may have resided largely near the surface of dust layers.

Table 6. Other dust specific activities from various locations in AVR (IAEA 1997).

\begin{tabular}{|l|c|c|c|c|c|}
\hline \multirow{2}{*}{ Point of extraction } & \multirow{2}{*}{ Year } & \multicolumn{4}{|c|}{ Concentration [Bq/g dust] } \\
\cline { 3 - 6 } & & $\mathbf{C o - 6 0}$ & $\mathbf{A g - 1 1 0 m}$ & $\mathbf{C s - 1 3 7}$ & Sr-90 \\
\hline \multirow{2}{*}{ Fuel Element Surfaces } & 1978 & $1.8 * 10^{6}$ & $5.4 * 10^{4}$ & $1.0 * 10^{6}$ & $2.7 * 10^{6}$ \\
\cline { 2 - 6 } & 1988 & $3.0^{*} 10^{4}$ & $5.1 * 10^{3}$ & $1.3 * 10^{5}$ & $9.0^{*} 10^{5}$ \\
\hline Steam generator & 1986 & $5.1 * 10^{6}$ & $2.7 * 10^{7}$ & $7.4 * 10^{7}$ & $3.6^{*} 10^{8}$ \\
\hline \multirow{2}{*}{ Cold gas zone } & 1978 & $6.7 * 10^{5}$ & $1.6 * 10^{6}$ & $3.0 * 10^{7}$ & $2.5 * 10^{8}$ \\
\hline & 1988 & $1.6 * 10^{6}$ & $6.4 * 10^{5}$ & $5.9 * 10^{6}$ & $5.0^{*} 10^{7}$ \\
\hline Fuel cycle system & 1978 & $7.4 * 10^{4}$ & $9.3 * 10^{4}$ & $1.5 * 10^{5}$ & - \\
\hline Shutdown rod & 1987 & $4.3 * 10^{5}$ & $1.9 * 10^{5}$ & $1.3 * 10^{7}$ & - \\
\hline
\end{tabular}

The size distribution of AVR dust was measured and found to largely contain submicron particles with a mean diameter of $0.6 \mu \mathrm{m}$, corresponding to a volume-weighted mean of 5 to $10 \mu \mathrm{m}$. The small size of particles is cited as a reason for the aforementioned small deposition per pass, and the size distribution did not appear to vary significantly with the location of the sample. Examples of measured AVR dust size distributions are shown in Figure 2.

VAMPYR-I was a 2.2-m-long, hot-gas sampling tube that operated for many years in order to measure plateout. Dust did not appear to significantly alter deposition profiles measured in VAMPYR-I. Activities on the dust filter were comparable to those of the cold-gas filter (Moorman 2008), and cesium and iodine deposition profiles were well described by the SPATRA code (IAEA 1997).

The VAMPYR-II experiment was installed later in life, and four experiments were carried out. VAMPYR-II was located such that neutron activation would not contribute to Ag-110m production, which had occurred in VAMPYR-I, and so that temperature could be better controlled and measured. The effect of dust on plate-out were apparently more pronounced in VAMPYR-II as deposition profiles (especially for cesium) were relatively uniform, but the profiles did not change significantly even with the addition of a dust filter in the final experiment. Thus, the cesium behavior remains unexplained. This experience appears similar to that in FSV, where it was concluded that dust was not being adequately filtered.

Based on the estimated quantity of $60 \mathrm{~kg}$ of dust in AVR and the various activity measurements, it was estimated that $8 \%$ of the released cesium and $11 \%$ of the released strontium were associated with dust (von der Decken \& Wawrzik 1990).

Another estimate has been put forth (Natesan, Purohit, \& Tam 2003) that, based on AVR data, 73\% of cesium is expected to plateout on metals and only $5 \%$ on dust. The details of that estimate were not known at the time of this writing.

In the PBMR safety analysis report, specific activities for Cs-134 and Cs-137 on dust from AVR are reported to be $6 \times 10^{4} \mathrm{~Bq} / \mathrm{g}$ and $2.6 \times 10^{5} \mathrm{~Bq} / \mathrm{g}$, respectively. 


\subsubsection{THTR}

Dust collected from moisture sensors in THTR had a high activity $\left(2 \times 10^{8} \mathrm{~Bq} / \mathrm{g}\right)$ because of the presence of Co-60, Zr-95, Hf-181, and Pa-233 (EPRI 2004), resulting from damage to spherical fuel elements by shutdown control rods inserted directly into the pebble bed (Baumer \& Kalinowski 1991). Subsequent measurements gave activities of $1.1 \times 10^{6} \mathrm{~Bq} / \mathrm{g}$ for Cs- $137,5.7 \times 10^{5} \mathrm{~Bq} / \mathrm{g}$ for Cs- 134 , and $5.0 \times 10^{5} \mathrm{~Bq} / \mathrm{g}$ for $\mathrm{Ag}-110 \mathrm{~m}$. ${ }^{\mathrm{d}}$ If these activities are assumed for the entire estimated dust inventory of $25 \mathrm{~kg}$, a core release fraction less than $10^{-6}$ is implied. Since it was expected to be much higher, especially because of the fuel failures, it was suggested that cesium must not have associated strongly with dust (EPRI 2004). Since, for a pebble bed reactor, THTR had a relatively small amount of dust present (because of a short operating time), it is possible that larger quantities could result in increasing cesium involvement with dust.

\subsubsection{Deposition Loops}

In the absence of dust, FPs are transported as vapors and plateout on surfaces in the reactor system. The surface concentration of an FP on such (clean) surfaces is limited either by mass transport or sorption isotherms, which relate the surface concentration to the partial pressure and temperature of the FP in the gas. Many experiments and codes treat this phenomenon (EPRI 2004; EPRI 2002; Hanson \& Bolin 2007) but few that have considered explicitly the effect of dust. In addition to the VAMPYR experiments in AVR discussed above, a deposition loop at General Atomics ran plateout and liftoff experiments (Hanson 1976), two of which included dust (one in which $1 / 2 \mathrm{~g}$ had been added, another in which the dust had been generated). The general conclusions of this work on dust were that:

- Deposition profiles were considerably flatter, and there was more scatter in the data, in the presence of dust

- Activity associated with dust was considerably easier to remove in a blow-down

- Results would be difficult to extrapolate to reactor conditions and scale.

On the last point, it was noted that partial pressures of FPs in the experiment were higher than would actually be encountered, that the dust size distribution may not be representative, and that FPs exhibiting a preference for deposition on metals may migrate to them over longer periods of time, even if initially deposited on dust.

None of the operating HTGRs nor those operated in the past experienced a significant and unintended depressurization. Under these circumstances, fission-product-laden dust can be expected to remobilize and be transported into the reactor building. Subsequent release from the reactor building is a function of the building structure and is largely a complex thermal-hydraulic problem that may be addressed with the experimental and computational quantification of FP retention factors. Such factors are functions of particle transport, deposition in multicompartment configurations, dead-end zones, etc., and require generic experiments to improve and validate CFD and lumped parameter codes. They should be included in a larger R\&D experimental program if HTGR FP releases to the building are expected to be significant.

\subsection{Recent and Ongoing Research}

The FPs of greatest concern that potentially interact with dust are iodine, cesium, strontium, and silver. As the various activity measurements discussed above would indicate, the extent to which each will interact with dust rather than metallic or other solid surfaces is highly uncertain. Though dust activities have been measured in many deposition loops, none of the available legacy data appear to have

d. Details of these measurements are presumably given in reference 5-68 of EPRI. (2004), in German. 
quantified the relative fractions of activity on dust versus solid surfaces as, say, a function of dust loading. There also seems to exist uncertainty as to whether or not certain FPs will interact with dust at all. For example, it is stated (Kissane 2009) that there is no evidence for interaction of silver and carbonaceous dust, though elsewhere (Westinghouse 2008) they have been reported to interact strongly. There appears to be almost no theoretical consideration of this topic, but what is available is briefly summarized here.

Some theoretical work has been carried out on the attachment of gaseous FP to dust particles (Skyrme 1985). The results indicate that FPs can be expected to attach to dust particles, that this is more likely to occur for smaller particle sizes, and that it happens rapidly: in most circumstances, the time constant for attachment to dust particles is shorter than that for attachment to surfaces. The size dependence indicates that small particles may carry a disproportionately large quantity of FPs. An example is given for a typical lognormal distribution of particle diameters, in which only $6 \%$ of the total mass is in particles below $0.5 \mu \mathrm{m}$ in diameter, but these particles carry $64 \%$ of the FPs.

However, it is expected that, during normal operation, only a vanishingly small fraction of dust present will be circulating (IAEA 1997); rather, it will be deposited on surfaces. In the event surfaces are largely covered with a thin dust layer, it stands to reason that most FPs would attach to this dust and not the underlying surface. The extent to which this occurs is obviously dependent on the amount of dust produced, its distribution under normal conditions, and the surface area that it presents, which, for irregular particles, may be large even for relatively light surface loadings.

Regardless of its location, the quantity of FPs carried by dust particles will depend not only on the composition of the dust, but also of the particular FP and its chemical form. Interaction models such as those described above, which depend on sticking probabilities that must be measured, should be based on experiments in which conditions are similar to those expected in NGNP such that FPs have the same chemistry. Speciation and chemical behavior of FPs appears to be relatively unknown (Kissane 2009; NRC 2008), and this may be an important prerequisite to meaningful dust analysis. FP adsorption on dust, whether circulating or deposited, will also increase with BET surface area. Graphite particles are irregular and can be expected to have a high surface area, but this does not appear to be well accounted for in previous analyses.

Some analysis of iodine (and other FP) sorption on dust has been considered (Wichner 1991) where it was assumed that the concentration of iodine on dust or metal surfaces was small relative to its maximum (a monolayer). In this regime, the surface concentration is a linear function of the partial pressure of the FP in the gas. If the relevant constants for the sorption isotherms are known for both dust and surface materials, and the available areas of each are known, the quantity adsorbed on surfaces and dust can be determined. An estimate for the Modular High Temperature Gas Reactor, based on Peach Bottom dust data, concluded that between 2.9 and $36.3 \%$ of iodine would be adsorbed on dust. The primary uncertainty is the available area of the dust. This appears not to have been characterized in Peach Bottom (or anywhere else); characterization will be necessary for models to be developed and verified.

Some consideration has also been given to interaction of dust and FPs in SPECTRA. A previous model (Westinghouse 2008) simply uses the measured activity per mass of dust based on AVR data, and the activity associated with dust is inferred from this estimate and the mechanistic calculation of the dust distribution. As the AVR measurement alone carried an order of magnitude uncertainty, and the dustbound activity is about an order of magnitude lower than the surface-bound activity predicted in (Westinghouse 2008), better estimates may dramatically change the distribution of FPs between solid surfaces and dust. A more mechanistic model for FP sorption on dust was recently implemented in SPECTRA (Stempniewicz, Winters, \& Casperson 2010), although references to detailed information on its development were not available to the author at the time of this writing. Still, there is significant uncertainty in the model because of the general lack of experiment data and high uncertainty in what data are available. 


\subsection{Gap Analysis}

Though previous measurements of dust-bound activity in HTGRs may provide a basis for bounding calculations, a better understanding of FP adsorption on dust is needed. Even for a dust free reactor, sorption isotherms for graphite and metals are a present research need. If large quantities of dust are expected in a pebble bed reactor, sorption on dusty surfaces will need to be considered. It will be important to understand the extent of dust coverage in this case. If it is only partial, metal surfaces will compete with dusty surfaces, and FPs might preferentially associate with one or the other. If dust production and transport is such that metal surfaces will all be coated with dust, FPs will have no opportunity to plateout on metals at all, and the importance of sorption on dust is obviously increased. If this is the expectation, dust sorption will need to be considered in the existing R\&D plan for FP adsorption.

\subsection{Planned Research}

A comprehensive plan for examining the effects of dust on fission product plateout and liftoff is already described in the Fuel Development and Qualification Program Plan (Petti 2010). Tasks outlined therein are:

- Task 3.5.7, Radionuclide Deposition Characteristics on Structural Metals. This task calls for measurement of sorption isotherms for $\mathrm{Cs}, \mathrm{Ag}$, $\mathrm{Te}$, and I at relevant partial pressures on primary circuit structural alloys. This is necessary to determine plateout behavior in the "dust-free" case.

- $\quad$ Task 3.5.8, Radionuclide Reentrainment Characteristics for Dry Depressurization. This task describes separate effects tests (out-of-pile) to measure lift-off of fission products from structural metals, the complement to Task 3.5.7. This is necessary to determine liftoff behavior in the "dust-free" case.

- Task 3.5.9, Plateout Distribution Validation Data. This task calls for an inpile loop to provide integral test data for code validation. The task as presently described includes examination of the effects of dust on radionuclide deposit.

- $\quad$ Task 3.5.10, Radionuclide Plateout and Reentrainment (Liftoff) Validation Data. As with Tasks 3.5.7 and 3.5.8, this task compliments Task 3.5.9, and considers liftoff of deposited radionuclides. This task calls specifically for tests with and without dust in an integral facility (inpile loop) to examine the effects of dust on FP liftoff/resuspension.

The research plan outlined above is sufficient to characterize dust/FP interaction in HTGRs. One area that requires clarification is what constitutes "representative conditions" for dust resuspension in Task 3.5.10. This is clarified with a new subtask in Section 8.4. 


\section{RESUSPENSION}

Dust deposited on surfaces during normal operation represents a potential source of FP release since it may be resuspended by an increase in flow rates that result in an accident scenario. It is potentially much easier to resuspend solid particles containing FPs than FPs adsorbed on clean surfaces; thus, the presence of dust could result in a greater release of radioactivity under the same accident conditions.

\subsection{Operational Experience}

Experiments on dust resuspension were carried out in AVR in 1986 (Areva 2011). With the reactor shut down, the circulator speed was quickly increased from 1,500 to 3,000 and 4,000 rpm (and maintained there) to simulate an accident transient. The resulting dust concentrations in the helium were determined from collections in the Cold Gas Filter, Dust Experiment, and VAMPYR-I. The result in each case was an immediate increase in the circulating dust concentration, which then decreased over time as shown in Figure 9.

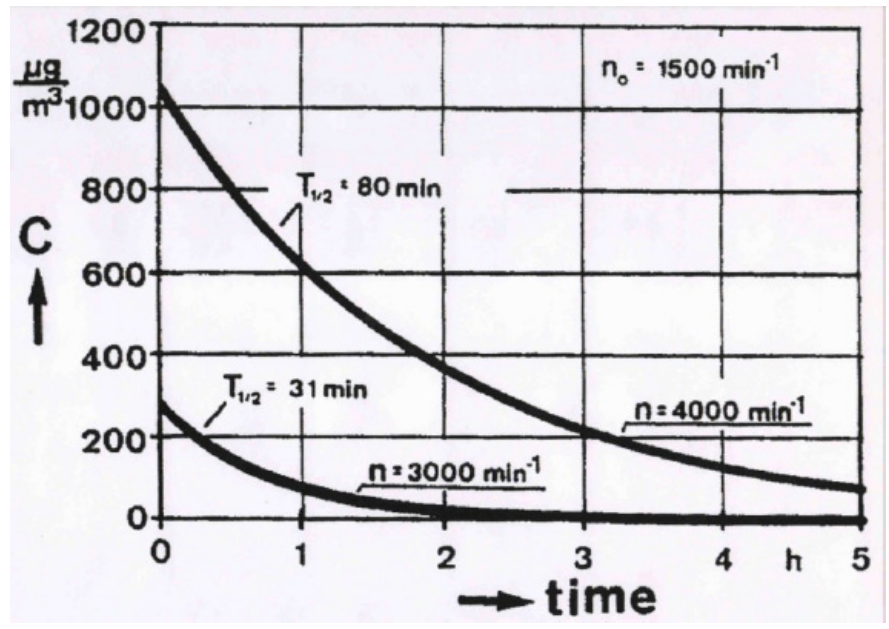

Figure 9. Circulating dust concentrations in the AVR resuspension experiments.

The typical circulating concentration of dust in the AVR was extremely low $\left(\sim 5 \mu \mathrm{g} / \mathrm{m}^{3}\right)$. Even though the AVR experiments at 3,000 and 4,000 rpm circulator speed resulted in increases to $\sim 280$ and $\sim 1050$ respectively, these quantities are still very small, representing less than $0.1 \%$ of the total dust inventory.

A recent reinvestigation of AVR dust deposits (Fachinger et al. 2008) lends some insight into the nature of AVR dust deposits. A section of pipe near the entrance to the helium purification system was removed to analyze dust deposits. These were found to be very tightly adhered to the surface: an insignificant quantity of material was obtained, even after knocking the pipe sections with a hammer. Ultimately, the layers had to be removed by scraping the pipe surface with a knife using considerable force as shown in Figure 10. The nature and formation of this crust is a key issue. If, as in the AVR, most of the generated dust adheres strongly to the pipes and other surfaces in the primary coolant loop, then the importance of dust as a FP vector is greatly reduced, becoming instead a radiological issue during decommissioning. 


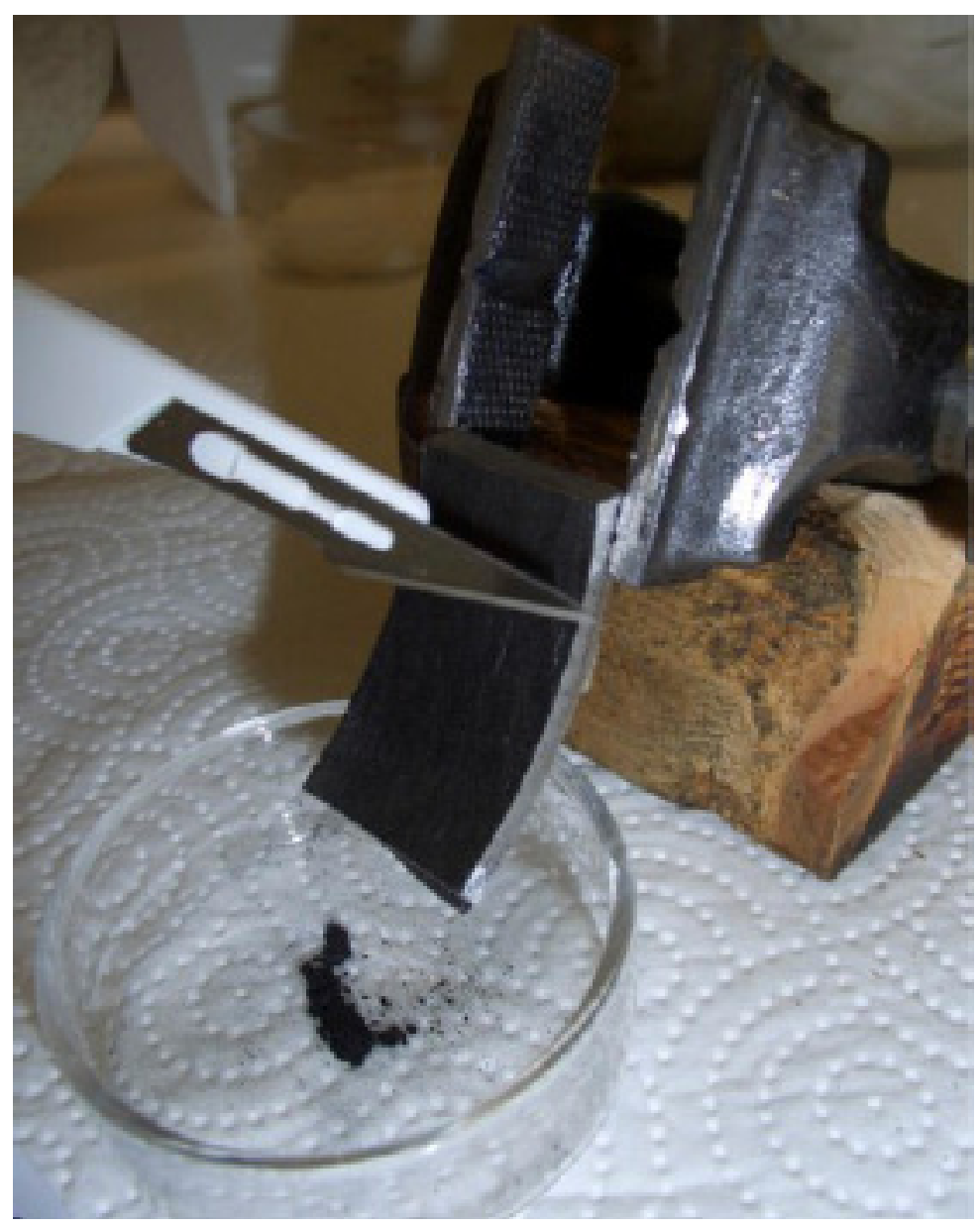

Figure 10. Removal of dust by scraping a section of AVR pipe (Fachinger et al. 2008).

\subsection{Recent and Ongoing Research}

Early attempts were made to model resuspension of dust using the shear ratio model (described in Wichner [1991]) used previously for lift-off of plated-out FPs. In the shear ratio model, resuspension is assumed to not occur under steady-state conditions, and the resuspended fraction is a function of the ratio of fluid shear during a transient to the fluid shear at steady-state (there is no time dependence). While a general trend of increasing resuspension with shear ratio was observed, data were scattered, and resuspension was generally influenced by other factors (Sawa, Nishimoto, \& Miyamoto 1992). Applied to dust, it stands to reason that one such parameter would be the particle size.

More sophisticated empirical models exist, such as those in the ECART code (Parozzi 1997). The ECART code calculates the force on particles resulting from several phenomena combined:

- Gravity

- Cohesion

- Frictional adhesion

- Fluid drag

- Turbulence 
The fractional resuspension rate in ECART is given by an empirical power law correlation with this resultant force. An example, including several sets of experiment data used to make the correlation, is shown in Figure 11.

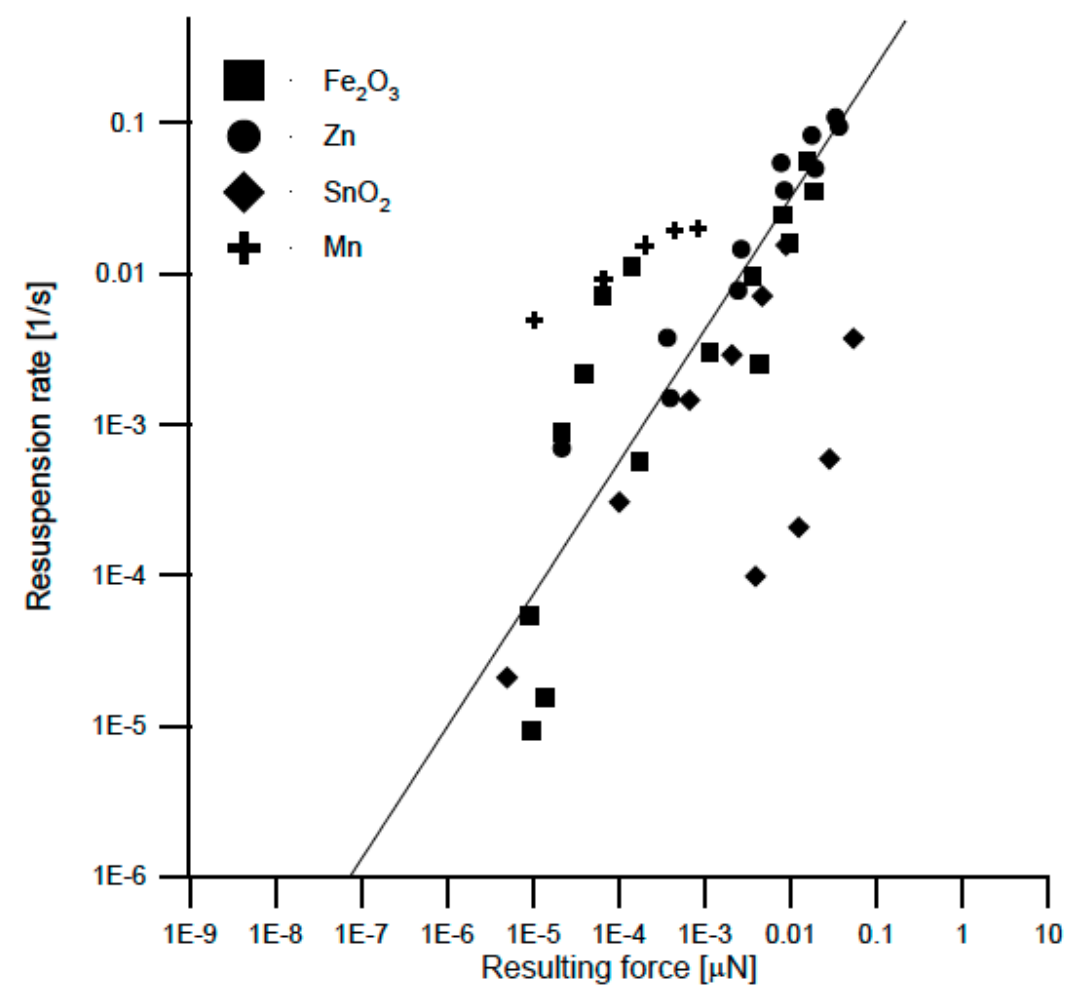

Figure 11. ECART correlation for the resuspension rate and experiment data (Parozzi 1997).

Another class of resuspension models has been developed more or less from first principles. These are similarly based also on the idea that turbulent bursts are largely responsible for resuspension, but they employ an Arrhenius law, rather than an empirical correlation, for the resuspension rate (Reeks, Reed, \& Hall 1988). The activation energy is determined by the adhesive energy and turbulent energy imparted to the particle. The model was refined by (Vainshtein, Ziskind, Fichman, \& Gutfinger 1997) to include rolling because of drag moments as the primary detachment mechanism; Reeks \& Hall (2001) later included both drag and lift forces. Both models are complicated by the fact that the particle sizes are on the order of the features of a rough surface. In this case, the turbulent flow forces are difficult to characterize, and most importantly, the particles are not spheres on a flat surface, for if they were, the adhesive energy would be well characterized. So instead, an entire distribution of surface adhesive forces is present. This is presumed to follow a lognormal distribution, and is therefore characterized by two parameters. Determining these parameters is challenging at best, and has not been done in previous resuspension tests except for the centrifuge experiments (Reeks \& Hall 2001).

Recognizing this difficulty, four sets of available experiment data were considered (Biasi, de los Reyes, Reeks, \& de Santi 2001) and a correlation for both lognormal parameters with the particle radius developed. With these correlations, the Reeks and Hall model reproduces all these experiments within $30 \%$. However, since the lognormal parameters characterize the surface, it is not clear why they should correlate with (only) the particle diameter.

Both the Vainshtein and Reeks and Hall resuspension models have been successfully implemented in SPECTRA (Stempniewicz, Komen, \& de With 2008) and MELCOR (Merrill \& Humrickhouse 2011); DAMD opts for a shear ratio model, though validation against experiments is still an open issue. The 
relatively little available data are sometimes difficult to interpret. Consider, as an example, the STORM experiments, which are described along with an ASTEC/SOPHAEROS model (Bujan, Ammirabile, Bieliauskas, \& Toth 2010). In these experiments, dust was deposited (primarily by thermophoresis) at low velocity, after which step changes in velocity were made, and the corresponding resuspended fraction was measured. Some difficulties are apparent:

- Test 9 had a similar initially deposited mass to Test 11 , despite significantly different temperature gradients (different deposition would be expected by thermophoresis)

- Test 10 had a similar final resuspended fraction to Test 11 , despite rather different final velocities ( $\sim 85$ vs $135 \mathrm{~m} / \mathrm{s})$

- Resuspended particles had larger diameters than when they deposited, implying some agglomeration at surfaces (de los Reyes, Capitao, \& De Santi 1999).

\subsection{Gap Analysis}

Resuspension is a complex phenomenon, and modeling it is challenging. Though approaches to resuspension modeling vary from completely empirical to highly theoretical, they seem to share a similar conception of deposited dust as relatively weakly adhered individual particles. Resuspension experiments have typically dealt with this kind of material. It is apparent that the AVR dust deposits were rather different; they formed hard crusts that were very immobile, as evidenced by resuspension experiments and end-of-life analysis. The immobility of AVR dust was apparently convincing in the licensing of HTR-module, and it is important to understand the basis for that argument. There do not appear to be any existing facilities suitable for investigation of formation (or lack thereof) of such layers.

\subsection{Planned Research}

The large quantity of dust that was present in AVR is the primary motivator for this examination of dust safety issues for HTGRs. Yet, it has been demonstrated that very little of this dust inventory was mobilizable, even in experiments designed to do so. Recent reinvestigations have shown that the dust in AVR had hardened into a crust on surfaces where it resided, and was extremely difficult to remove. This material is altogether different than the loose particulate typically employed in dust resuspension experiments. Understanding this phenomenon was identified as a high priority issue at the Dust Issues Assessment Meeting.

A series of R\&D tasks related to fission product plateout and liftoff, which incorporates the effects of dust, was outlined in Section 7. The Fuel Development and Qualification Program Plan (Petti 2010) calls for tests at "representative conditions," and a new subtask is necessary to elucidate what is representative for dust layers; loose dust introduced to an otherwise dust-free experiment may not be representative.

An experiment should be carried out under this new subtask that examines the effects of dust deposit aging. The experiment will consist of a high temperature and pressure helium loop (NGNP relevant conditions) in which dust is continually circulating. Surface samples should be examined at regular intervals to investigate the nature of dust deposits over time. Effects to be considered in this experiment include:

- Control of relevant impurities ( $\mathrm{CO}, \mathrm{CO}_{2}, \mathrm{H}_{2}, \mathrm{H}_{2} \mathrm{O}$, etc.)

- Consideration of oil ingress. It was suggested that oil ingress in AVR contributed in some way to the crust formation, but this is presently speculative. The absence of oil in an NGNP may lead to a different morphology of deposited layers.

- Resuspension of deposits because of increases in flow rate. These should be carried out on samples of various ages to determine the effect of the aging on resuspension. 
Data collected under this subtask will identify representative conditions for FP plateout and liftoff tests described in Section 7, and provide validation data for dust resuspension models.

It should be noted that the long duration likely required to examine aging effects experimentally would attach a high cost to this subtask. 


\section{EXPLOSION OF DUST IN CONFINEMENT}

It has been noted that large amounts of suspended graphite dust may present a combustion or explosion hazard, similar to that of grain or coal dust. But before proceeding, it is useful to clarify some terminology. Oxidation implies, simply, a chemical reaction of material (graphite) with oxygen. Such a reaction that is self-sustaining is described as burning or combustion. A deflagration or explosion occurs when a mixture of dust and gas permits rapid flame propagation; these are the safety issues we are primarily concerned with here.

\subsection{Operational Experience}

Though graphite can certainly oxidize, it is extremely difficult to burn because it is a near-perfect black-body radiator with a high heat capacity. Graphite is used in some applications where it reaches extremely high temperatures, including electrodes of arc lamps used to melt steel as shown in Figure 12.

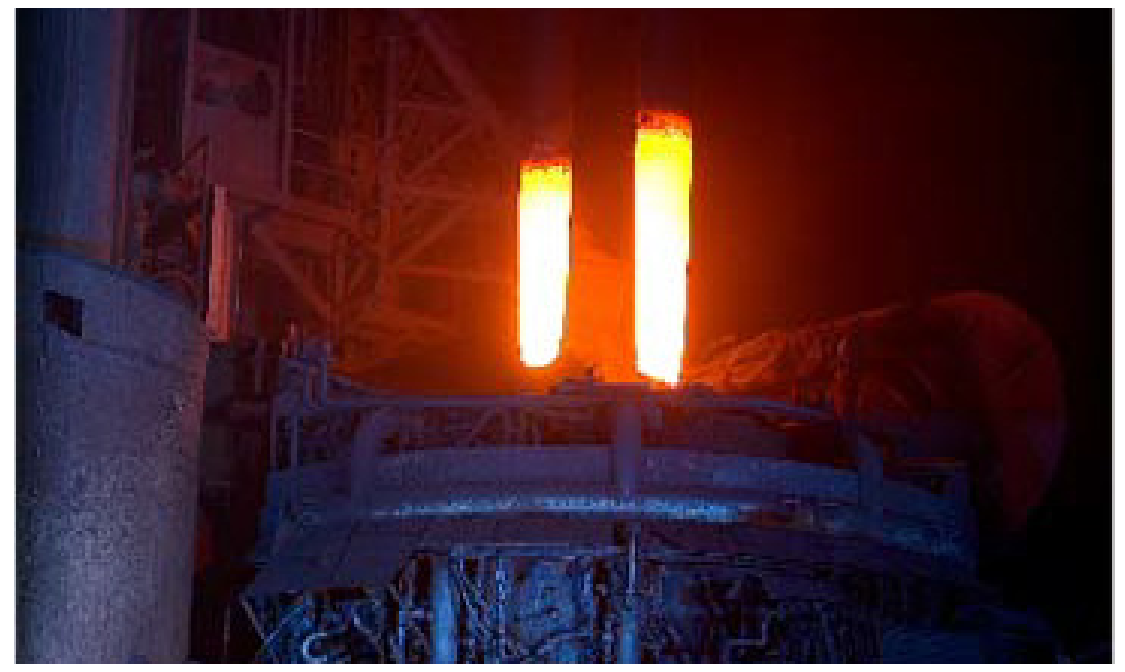

Figure 12. Graphite electrodes being inserted into a scrap metal furnace.

Red-hot graphite is often air-cooled during its manufacture, but graphite structures are never observed to burn, although oxidation may occur. Facilities that manufacture graphite components produce a considerable amount of dust in machining, but there has never been a dust explosion in the history of graphite manufacture. In fact, graphite powder is the primary component in some Class D fire extinguishers, used to extinguish lithium, magnesium, sodium, or other metal fires. The graphite powder smothers the fire, and acts as a heat sink.

The issue of explosive graphite dust, among other graphite safety issues, has been considered previously in support of decommissioning of graphite-moderated reactors such as the Winsdscale Piles in the United Kingdom (IAEA 2006; EPRI 2006). For such an explosion to occur, the following conditions must be present:

- The dust must be combustible

- The dust must be resuspended or mobilized

- The dust/gas mixture must contain sufficient oxygen

- An ignition source of sufficient energy must be present. 
Nuclear graphite dust is much less combustible than, for example, coal dust; volatiles are the real contributors to coal dust explosions. Nuclear graphites, on the other hand, will have few impurities. Testing in support of the aforementioned decommissioning work found that nuclear graphite dusts must fall within a particular range of size and concentration to become explosive, and even then they are only weakly so. For particle sizes between 1.7 and $2.2 \mu \mathrm{m}$, dust mass concentrations of 200 to $2,000 \mathrm{~g} / \mathrm{m}^{3}$ and oxygen concentrations of over $50 \%$ were needed to initiate an explosion (IAEA 2006). Particle sizes $<0.3$ $\mu \mathrm{m}$ were needed to create an explosion in air; larger particles essentially act as heat sinks and are not an explosive hazard. The conclusion of this and other testing (IAEA 2006; EPRI 2006) was that there was no risk of graphite dust exploding.

\subsection{Recent and Ongoing Research}

Considerable amounts of dust are also generated in magnetic fusion devices because of plasmasurface interactions. The presence of radioactive materials in future devices such as ITER has prompted similar investigations of dust in general, and dust explosions in particular, for fusion. Since some plasmafacing components will be carbon fiber composites, these explosion tests have included graphite.

Explosion testing results (Denkevits \& Dorofeev 2005) are more or less consistent with those described in the preceding section: nominal $4 \mu \mathrm{m}$ dust in air could explode at concentrations of 125 to $500 \mathrm{~g} / \mathrm{m}^{3}$, but only for ignition energies of $2 \mathrm{~kJ} .1 \mathrm{~kJ}$ igniters could not initiate an explosion; larger $(\sim 32 \mu \mathrm{m})$ particles could not be ignited at any ignition energy.

A graphite dust explosion experiment, funded by the Nuclear Energy University Program in support of VHTR safety is presently under construction at the University of Idaho. Data are not yet available from this experiment.

\subsection{Gap Analysis}

Extensive research has identified conditions under which a graphite dust explosion may occur. These are not likely to occur in a gas reactor accident scenario:

- Circulating dust concentrations $\left(\sim 5\right.$ to $\left.\sim 1,000 \mu \mathrm{g} / \mathrm{m}^{3}\right)$ are many orders of magnitude below what is required for an explosion $\left(\sim 1 \mathrm{~kg} / \mathrm{m}^{3}\right)$

- The dust/gas mixture will be poor in oxygen (relative to air) because of the large quantities of helium present

- An ignition source of sufficient energy must be present.

Based on the available data, the risk of dust explosion is extremely remote, and this may be regarded as a low priority for subsequent research.

Other safety issues related to graphite oxidation include production of combustible gases (Kroeger 1989; Palmer, Sibulkin, Strehlow, \& Yang 1978) and liberation of FPs. Provided circulating quantities of dust are indeed small, these issues are only important for structural graphite, not dust.

\subsection{Planned Research}

Since it has been determined that the risk of a graphite dust explosion is extremely remote, no new research in this area should be planned. As noted previously, (nonexplosive) dust oxidation, as with structural graphite oxidation, may contribute to liberation of fission products. Oxidation rates are covered in the existing Graphite Technology Development Plan (Windes 2007). 


\section{CONCLUSION AND RECOMMENDATIONS}

Dust in HTGRs is one of those topics that defy simple categorization as either a fuel, material, or thermal fluid phenomenon and thus a multidisciplinary approach is required to address it in its entirety. For the most part, however, dust appears to have an impact only as an FP transport vector. The need to resolve dust issues is driven by the magnitude of the effect it may have on the mechanistic source term. This review of Dust-affected safety issues for HTGRs is a culmination of a literature review, the input from the vendors through DDNs and formal meetings among HTGR dust experts. From this input, the following observations and conclusions can be drawn:

1. Dust is generated in pebble bed reactors (as observed in AVR) primarily from abrasion between fuel spheres and the fuel handling system, while prismatic core reactors are not expected to generate significant amounts of dust.

2. As a first order approximation, carbonaceous dust is considered to behave in a manner similar to aerosol particles insofar as transport properties are concerned, but confirmatory data on dust transport characteristics are needed to validate this assumption.

3. Most of the dust in AVR was deposited as a hardened crust in the interior surfaces of the primary coolant loop. Most ( $>97 \%$ ) of this crust remained immobilized even while the loop was dismantled. It is an open question as to how this crust was formed and if the dust in modern HTGRs will be similarly immobilized.

4. Logistically, FP transport under clean (dust free) conditions should be characterized first in order to provide a baseline for judging the significant effects of dust. Major investigations of dust behavior should be conducted in concert with experiments that address the mechanistic source term, which are a part of the existing AGR program. Small-scale separate effects experiments and analyses can be focused on specific attributes of the overall dust issue.

5. Exploding dust is unlikely to be a significant risk in high temperature reactors, but the oxidation of circulating dust in this high temperature environment may have a consequential effect on FP remobilization.

The extent to which dust presents a safety issue in HTGRs depends first upon how much dust is in the system. If preliminary research indicates that significant amounts of dust will be generated, it is important to understand the affinity of dust for surfaces in the primary coolant loop and how easily the dust is remobilized under transient conditions. In contrast with previous separate effects experiments and modeling efforts, AVR dust formed an extremely immobile crust, and it is important to understand why. Finally, if significant amounts of dust are remobilized, it becomes necessary to understand the affinity of FPs for dust versus metal surfaces. This will require the generation of sorption isotherms for dust layers or dusty surfaces. These three questions, in that logical order, must be the focus of future research efforts.

Dust research within the NGNP R\&D Program should be conducted as part of the broader investigation of FP release, transport, and deposition. Many elements of dust research are also appropriate activities for Department of Energy-funded university research in support of NGNP. Finally, international research efforts such as the DUSTIN project (Lind, Güntay, Dehbi, Liao, \& Rycroft 2010) should be leveraged whenever possible to fill in gaps not captured in the current U.S. effort. 


\section{REFERENCES}

Ammar, Y., Lind, T., Reeks, M., \& Dehbi, A. (2007). Identification of the mechanisms for the break up of aerosol agglomerates in a PWR steam generator tube rupture. 6th International Conference on Multiphase Flow (ICMF).

Areva. (2008). NGNP Conceptual Design DDN/PIRT Reconciliation. Document No. 12-9102279-001.

Areva. (2011). Pebble Bed Reactor Technology Readiness Study. Document No. 12-9151714-000.

Ball, S. J. (2008). Next Generation Nuclear Plant GAP Analysis Report. ORNL/TM-2007/228.

Baumer, R., \& Kalinowski, I. (1991). THTR Commissioning and Operating Experience. Energy, 16 (12), 59-70.

Baumer, R., Kalinowski, I., Rohler, E., Schoning, J., \& Wachholz, W. (1990). Construction and operating experience with the 300-MW THTR nuclear power plant. Nuclear Engineering and Design , 121, 155-166.

Biasi, L., de los Reyes, A., Reeks, M. W., \& de Santi, G. F. (2001). Use of a simple model for the interpretation of experimental data on particle resuspension in turbulent flows. Aerosol Science, 32, $1175-1200$.

Bujan, A., Ammirabile, L., Bieliauskas, A., \& Toth, B. (2010). ASTEC V1.3 code SOPHAEROS module validation using the STORM experiments. Progress in Nuclear Energy, 52, 777-788.

Burnette, R. D. (1982). Radiochemical analysis of the first plateout probe from the Fort St. Vrain hightemperature gas-cooled reactor. GA-A16764.

Castelo, A., Capitao, F. A., \& Santi, G. International Standard Problem 40 Aerosol Deposition and Resuspension Final Comparison Report. European Commission Joint Research Centre Report EUR $18708 \mathrm{EN}$.

Clement, C. F., \& Harrison, R. G. (2000). Enhanced Localised Charging of Radioactive Aerosols. Journal of Aerosol Science, 31 (3), 363-378.

Cogliati, J. J., \& Ougouag, A. M. (2010). Dust Production Model for HTR-10.

Cogliati, J. J., \& Ougouag, A. M. (2008). Pebble Bed Reactor Dust Production Model. HTR-2008.

Cogliati, J. J., Ougouag, A. M., \& Ortensi, J. (2010). Preliminary Pebble Bed Reactor Core Dust Production Literature Review.

de los Reyes, A., Capitao, J. A., \& De Santi, G. (1999). International Standard Problem 40: Aerosol Deposition and Resuspension. NEA/CSNI/R(99)4.

Denkevits, A., \& Dorofeev, S. (2005). Dust explosion hazard in ITER: Explosion indices of fine graphite and tungsten dusts and their mixtures. Fusion Engineering and Design, 75-79, 1135-1139.

Dyer, F. F., Wichner, R. P., Martin, W. J., \& de Nordwall, H. J. (1977). Distribution of RadioNuclides in the Peach Bottom HTGR Primary Circuit During Core 2 Operation. ORNL-5188.

EPRI. (2004). A Review of Radionuclide Release from HTGR Cores During Normal Operation. No. 1009382. 
EPRI. (2006). Graphite Decommissioning: Options for Graphite Treatment, Recycling, or Disposal, including a discussion of Safety-Related Issues. No. 1013091.

EPRI. (2002). Plateout Phenomena in Direct-Cycle High Temperature Gas-Cooled Reactors. No. 1003387.

Fachinger, J., Barnert, H., Kummer, A., Caspary, G., Seubert, M., Koster, A., et al. (2008). Examination of dust in AVR pipe components. Proceedings of the 4th International Topical Meeting on High Temperature Reactor Technology (HTR-2008), (p. 58033). Washington, D.C.

Gallego, N. C., Burchell, T. D., \& Srinivasan, M. (2009). Milestone Report on the "Workshop on Nuclear Graphite Research". ORNL/NRC/LTR-09/03, Oak Ridge National Laboratory.

Gauntt, R. O. MELCOR Computer Code Manuals Vol. 1: Primer and Users' Guide Version 1.8.6. NUREG/CR-6119, Vol. 1, Rev. 3, Sandia National Laboratory.

General Atomics. (2008). Reconciliation of NGNP DDNs with NRC PIRTs. GA Document No. PC000570.

Gottaut, H., \& Kruger, K. (1990). Results of Experiments at the AVR reactor. Nuclear Engineering and Design , 121, 143-153.

Gougar, H. (2010). Next Generation Nuclear Plant Methods Technical Program Plan. PLN-2498.

Hanson, D. L. (2008). Engineering Services for the Next Generation Nuclear Plant (NGNP) with Hydrogen Production. GA-911117.

Hanson, D. L. (1976). Results of the General Atomic Deposition Loop Program. GA-A13140.

Hanson, D. L., \& Bolin, J. M. (2007). Radionuclide Transport in a Vented Low-Pressure Confinement. GA Document No. PC-000541.

Hanson, D. L., Baldwin, N. L., \& Selph, W. E. (1976). Gamma Scanning the Primary Circuit of the Peach Bottom HTGR. GA-A14161.

IAEA. (2008). Accident Analysis for Nuclear Power Plants with Modular High Temperature Gas Cooled Reactors. Safety Reports Series No. 54.

IAEA. (2006). Characterization, Treatment and Conditioning of Radioactive Graphite from Decommissioning of Nuclear Reactors. TECDOC-1521.

IAEA. (1997). Fuel Performance and fission product behaviour in gas cooled reactors. TECDOC-978.

INL. (2010). Mechanistic Source Terms White Paper. INL/EXT-10-17997.

Kissane, M. P. (2009). A review of radionuclide behaviour in the primary system of a very-hightemperature reactor. Nuclear Engineering and Design , 239, 3076-3091.

Kroeger, P. G. (1989). Safety Evaluation of MHTGR Licensing Basis Accident Scenarios. NUREG/CR5261.

Lind, T., Güntay, S., Dehbi, A., Liao, Y., \& Rycroft, C. (2010). PSI Project on HTR Dust Generation and Transport. Proceedings of HTR-2010, Paper 137. Prague.

Loyalka, S. K. (2010). NERI Project number 08-043 Quarterly Progress Report. 
MELCOR. (2005). Computer Code Manuals. NUREG/CR-6119.

Merrill, B. J. (2010). Applicability of MELCOR for Use in Analyzing Air Ingress Accidents in a South African Pebble Bed Modular Reactor (PBMR). INL/EXT-10-17658, INL.

Merrill, B. J., \& Humrickhouse, P. W. (2011). Aerosol Resuspension model for MELCOR for fusion and very high temperature reactor applications. INL/EXT-10-19683, INL.

Merrill, B. J., Humrickhouse, P. W., \& Moore, R. L. (2009). A Comparison of Modifications to MELCOR Versions 1.8.2 and 1.8.6 for ITER Safety Analysis. INL/EXT-09-16715, INL.

Moorman, R. (2008). Fission Product Transport and Source Terms in HTRs: Experience from AVR Pebble Bed Reactor. Science and Technology of Nuclear Installations , 2008, 597491.

Natesan, K., Purohit, A., \& Tam, S. W. (2003). Materials Behavior in HTGR Environments. NUREG/CR6824.

NEA. (2009). Experimental Facilities for Gas-cooled Reactor Safety Studies. NEA/CSNI/R(2009)8.

NEA. (2009). State-of-the-Art Report on Nuclear Aerosols. NEA/CSNI/R(2009)5.

Nieder, R. (1990). Schlußfolgerungen für die HTR-Chemie aus 21 Jahren Betrieb des AVR-Reaktors (Conclusions about HTGR Chemistry from 21 Years of AVR Operation). Chemie im Kraftwerk (Chemistry in Power Plants), 133-137.

NRC. (2008). Next Generation Nuclear Plant Phenomena Identification and Ranking Tables (PIRTs) Volume 3: Fission-Product Transport and Dose PIRTs. U.S. NRC.

NRI. (2010). Unpublished summary of HTHL.

Palmer, H. B., Sibulkin, M., Strehlow, R. A., \& Yang, C. H. (1978). An Appraisal of Possible Combustion Hazards Associated with a High-Temperature Gas-Cooled Reactor. BNL-NUREG50764.

Parozzi, F. (1997). ECART User Manual Part 2: Code Structure and Theory. Milan: ENEL Nuclear Energy Division.

PBMR. Safety Analysis Report.

Reeks, M. W., \& Hall, D. (2001). Kinetic models for particle resuspension in turbulent flows: theory and measurement. Aerosol Science, 32, 1-31.

Reeks, M. W., Reed, J. R., \& Hall, D. (1988). The resuspension of small particles by turbulent flow. Journal of Physics D , 21, 574-589.

Sawa, K., Nishimoto, T., \& Miyamoto, Y. (1992). Experimental Study of Dust Behavior during Depressurization. Journal of Nuclear Science and Technology , 29 (10), 1018-1025.

Petti, D. (2010). Techical Program Plan for the Next Generation Nuclear Plant/Advanced Gas Reactor Fuel Development and Qualification Program. PLN-3636.

Skyrme, G. (1985). Attachment of gaseous fission products to aerosols. IWGGCR-13: Fission product release and transport in gas-cooled reactors, (pp. 262-274).

Sparks, C. J., Specht, E. D., Easton, D. S., Beahm, E. C., Mailen, J. C., Sherrow, S. A., et al. (1991). Characterization of Ft. St. Vrain Reactor Dust. ORNL/NPR-90/12. 
Stempniewicz, M. M., Komen, E. M., \& de With, A. (2008). Model of particle resuspension in turbulent flows. Nuclear Engineering and Design , 238, 2943-2959.

Stempniewicz, M. M., Winters, L., \& Casperson, S. A. (2010). Analysis of Dust and Fission Products in A Pebble Bed NGNP. Proceedings of HTR-2010, (p. Paper 270). Prague.

Stoker, C. C., Olivier, L. D., Stassen, E., Reitsma, F., \& van der Merwe, J. J. (2010). PBMR radionuclide source term analysis validation based on AVR operating experience. Nuclear Engineering and Design, 240, 2466-2484.

Vainshtein, P., Ziskind, B., Fichman, M., \& Gutfinger, C. (1997). Kinetic model of particle resuspension by a drag force. Physical Review Letters , 78 (3), 551-554.

von der Decken, C.-B., \& Wawrzik, U. (1990). Dust and Activity Behavior. In AVR: Experimental HTR (pp. 259-275). Springer.

Westinghouse. (2009). Design Data Needs (DDNs) Reconciliation against PIRTs. NGNP-CDWP TIDDN.

Westinghouse. (2008). NGNP and Hydrogen Production Preconceptual Design Study. NGNP-NHS 50$\mathrm{CC}$.

Wichner, R. P. (1991). Fission Product Plateout and Liftoff in the MHTGR Primary System: A Review. NUREG/CR-5647.

Windes, W., Burchell, T., and Bratton, R. (2010). Graphite Technology Development Plan. INL/EXT-0713165.

Young, M. F. (2010). MELCOR Development for HTGR Applications. 
Appendix A

\section{Experimental Facilities}




\section{Appendix A-Experimental Facilities}

Some experimental facilities for gas cooled reactor safety studies have been summarized recently (NEA 2009). This appendix summarizes the facilities suitable for investigating the various dust phenomena discussed in this report.

\section{A-1. High Temperature Helium Loop}

The high temperature helium loop (HTHL) at NRI in the Czech Republic is primarily designed to investigate corrosion and irradiation of high temperature gas-cooled reactor (HTGR) materials. It is a helium loop that can operate up to $7 \mathrm{MPa}$ and $900^{\circ} \mathrm{C}$. Impurities can be added to the helium in controlled quantities to investigate corrosion and coolant chemistry.

While HTHL was identified (NEA 2009) as an experimental facility for investigation of aerosol growth, elsewhere (NRI 2010) it has been stated that the HTHL is not designed or suitable for dust and fission product transport experiments.

\section{A-2. THAI}

The THAI facility (operated by Becker Technologies GmbH) in Germany is a technical scale experiment facility for reactor containment safety studies. It is a $60 \mathrm{~m}^{3}$ multicompartment vessel $\sim 9 \mathrm{~m}$ high and $\sim 3 \mathrm{~m}$ in diameter. The compartments and connecting ducts can be arranged as necessary to model specific configurations of interest, and helium and dust can be introduced where desired.

Instrumentation in THAI includes 2-D/3-D particle image velocimetry and laser Doppler anemometry for flow measurements, iodine-123 radio-tracing, mass spectrometry, and heat conductivity sensors for gas concentration measurements. Impactors, photometers, filters, and deposition coupons are available for aerosol measurements.

\section{A-3. High Temperature Engineering Test Reactor}

The High Temperature Engineering Test Reactor (HTTR) presently operating in Japan includes monitoring of aerosol and doses in the reactor building and offsite. No serious releases have been found to date. Examination of dust on coolant filters may be possible when they are changed during maintenance. A plateout probe experiment is planned, but does not appear to be designed to consider dust effects. While the HTTR may be available for dust related experiments, it is not clear if any are currently planned.

\section{A-4. Paul Scherrer Institute}

The Paul Scherrer Institute (PSI) in Switzerland is planning an experimental campaign to investigate dust generation and transport for very high temperature reactors (Lind, Güntay, Dehbi, Liao, \& Rycroft 2010). Both integral and separate effects experiments are planned. The integral experiment will consist of both a core simulator (both pebble bed and prismatic experiments are planned) and a steam generator/heat exchanger. A variety of dust characteristics and transport phenomena will be investigated, including:

- The quantity of dust generated in a pebble bed design

- Dust characteristics, including size, BET surface area, and composition (via both in and ex situ techniques)

- The distribution of deposited dust throughout the experiment

- Resuspension of dust during rapid depressurization. 
A separate effects experiment is discussed that would examine the aging of deposits (e.g., the AVR dust crust. It should be noted that the facility will not be able to accommodate radioactive materials or fission products.

\section{A-5. Other experiments}

With the exception of the planned facility at PSI, the experiments described by NEA (2009) appear primarily to focus on transporting dust and thermal hydraulics, and not on the generation, interaction with fission products, or explosions.

The dust generation experiments ongoing at the University of Missouri have been previously mentioned. A dust production experiment is planned or operating at the University of Idaho, but details of this facility are not presently known.

Dust found in representative samples should be well characterized, which has not historically been done. Well-established methods of sampling and characterizing dust size distributions have been developed for fusion, and are available at Idaho National Laboratory (INL) and likely other fusion facilities.

Fission product sorption on dust is another issue of importance that has not been thoroughly studied. It is likely to require use of actual fission products, given the small quantity involved and the associated difficulties in measurement. A fission product sorption experiment is being discussed at INL to which dust might eventually be added, but discussions are only in the very earliest stages.

In the event explosion testing is warranted, established facilities for fusion dust might be leveraged. Such a facility, using a standard 20 liter spherical explosion chamber, will come online at INL in 2011. 
Appendix B

\section{Modeling Tools}




\section{Appendix B-Modeling Tools}

The section summarizes basic aerosol and dust capabilities of several codes, focusing particularly on three that have been or are being developed specifically for gas reactor applications: SPECTRA (Sophisticated Plant Evaluation Code for Thermal-hydraulic Response Assessment), MELCOR, and DAMD (Dust and Activity Migration and Distribution).

\section{B-1. SPECTRA}

The SPECTRA code is an accident analysis code developed at NRG in the Netherlands (Westinghouse 2008). Though applicable to light water reactors (LWRs) and conventional power plants, development for gas reactor applications is ongoing. SPECTRA has recently been applied, for example, to the South African demonstration power plant.

SPECTRA is divided into a number of packages containing models for various plant phenomena. A model consists of a system of control volumes and junctions, similar to MELCOR or CONTAIN.

The code structure is described in terms of Packages, containing Models that perform similar tasks. The modeling approach is based on the Control Volume concept. A model of a certain physical system consists of Control Volumes, connected by junctions. The approach is similar to that taken in, for example, CONTAIN or MELCOR.

The Radioactive Particle Transport Package treats release of fission products and transport of aerosols. Radioactive chains of fission products are tracked, and mechanistic models for various aerosol transport phenomena are present, including:

- Gravitational settling (with Cunningham slip correction factor)

- Thermophoresis

- Brownian diffusion

- Turbulent deposition (inertial impaction, diffusion impaction, diffusional deposition)

- Inertial impaction

- Agglomeration of circulating (Brownian, gravitational and turbulent) and deposited dust

- Resuspension (Rock'n Roll and Vainsthein resuspension models).

Resuspension can also be treated with a parametric model and user-defined coefficients, presumably obtained from some experiment data.

In addition to models for fission product condensation and sorption, a model for sorption on dust particles is included.

\section{B-2. MELCOR}

MELCOR is a fully integrated, engineering-level computer code that predicts the progression of severe accidents in LWR nuclear power plants (MELCOR 2005). MELCOR is being developed at the Sandia National Laboratories (SNL) for the U.S. Nuclear Regulatory Commission. This code calculates a spectrum of accident phenomena, some of which are: reactor cooling system and containment fluid flow, heat transfer, and aerosol transport. Though originally designed for LWR accident modeling, it is now being actively developed for gas reactor applications. 
MELCOR solves nonequilibrium conservation equations for mass, momentum, and energy (different phase temperatures and velocities) for the liquid and vapor phase of water. Noncondensable gases can be included in the vapor atmosphere. Flow between confinement volumes includes the effect of frictional/momentum form losses and sonically limited (choked) fluid flow. MELCOR treats hydrogen and carbon monoxide combustion, including predictions of ignition conditions and burn rates of hydrogen-carbon monoxide-steam-air-mixtures.

Aerosol transport and deposition is treated by MELCOR to allow the user to track the movement of radioactive-laden aerosol particles (e.g., dust or particulate) within a given facility, and even their eventual release to the environment. This is handled by the radionuclide package of MELCOR. Since it is intended as a tool for severe accident analysis, it must account for the release and transport of radioactive fission products that, upon release to the environment, become a source term, which is one major product of the overall accident calculation in MELCOR. Source terms are then used to calculate consequences as the end product of the safety assessment.

While many MECLOR radionuclide package transport models are specifically designed for LWRs, the source term portion of MELCOR can run without invoking LWR specific models. For example, the radionuclide package allows the user to directly specify an aerosol source term such as an aerosol source term of carbon dust. These time-dependent aerosol source terms can have a user-defined size distribution, a uniform source distribution with respect to log diameter (log uniform), or a log-normal distribution with respect to the log of particle diameter.

The MELCOR radionuclide package model considers aerosol transport processes that allow the prediction of aerosols movement between rooms of a confinement building. The MELCOR aerosol dynamics model is based on MAEROS, a multisection, multicomponent aerosol dynamics code. Aerosols move by Brownian motion, in addition to bulk flow within the coolant, and can be deposited directly on surfaces such as heat structures and water pools, by gravitational settling, diffusiophersis, or thermophoresis. Aerosols can grow in size by agglomeration and steam condensation. However, MELCOR automatically models aerosol behavior in a dry atmosphere, like that expected in a very high temperature reactor (VHTR), when water is not included in the calculation.

Chemistry effects can be simulated in MELCOR through the class reaction and class transfer models, which are controlled entirely by user-specified parameters. The class reaction process uses a first-order reaction equation to simulate reversible chemical reactions. With these models, phenomena such as adsorption, chemisorption, and other important chemical reactions can be simulated. Only fission product vapors are currently treated with these mechanisms. Chemisorption of radionuclides on surfaces can also be simulated with the chemisorption model, for example the chemisorption of fission product gases on carbon dust.

A number of additions have recently been made to MELCOR for application to gas reactor systems. These include models specific to thermal hydraulics and heat transfer of pebble bed and prismatic reactors, models for release and diffusion of fission products through tristructural isotropic (TRISO) fuel, including intact and failed particles, and external uranium contamination (Young 2010). Fuel failure fractions and dust generation rates are determined by user inputs, which will presumably be obtained from other codes or experiment data as it becomes available. Dust resuspension and dust-fission product interaction models are planned at SNL (Young 2010).

Some modifications (many originally intended for fusion safety analyses) have also been made to MELCOR at INL. These include turbulent and inertial aerosol deposition, resuspension (Merrill and Humrickhouse, 2011), binary diffusion, graphite oxidation, and tritium transport. INL and SNL MELCOR development efforts for VHTRs are in the process of being integrated. 


\section{B-3. DAMD}

DAMD models the distribution of dust in fission products in an HTGR, and was developed specifically for pebble bed applications. It has been used to model these phenomena in the South African demonstration power plant design and Arbeitsgemeinschaft Versuchsreaktor (AVR; Stoker, Olivier, Stassen, Reitsma, \& van der Merwe 2010).

Though also based on a control-volume thermal hydraulic approach, in some ways DAMD treats dust and activity migration differently than SPECTRA or MELCOR. While some transport phenomena (gravity, drag) are considered explicitly, many deposition phenomena are lumped into empirical parameters that are assumed to be constants. These constants are set primarily based on AVR data, and plateout distributions from VAMPYR have been successfully modeled with DAMD that compare favorably to predictions of the RADAX code. Some analysis of other systems is probably warranted in order to establish the generality of the integral constants employed in the code.

\section{B-4. ECART}

ECART (ENEL Code for the Analysis or Radionuclide Transport; Parozzi 1997) is an integral systems code originally designed in the late 1980s for analysis of LWR accidents. More recently, ECART has been used to analyze fusion reactors (ITER) and industrial facilities. It treats a wide variety of aerosol phenomena, including:

- Deposition by:

- Gravitational settling (Stokes and non-Stokes regimes)

- Impaction (caused by turbulence and pipe bends)

- Diffusion

- Thermophoresis

- Diffusiophoresis

- Agglomeration:

- Brownian

- Gravitational

- Turbulent

- Resuspension (empirical model, based on experiments)

- Shape Factors:

- Aerodynamic

- Collisional.

\section{B-5. NGNP code use}

MELCOR is presently under development at SNL and Idaho National Laboratory for gas reactor applications. It is anticipated that MELCOR will be the primary systems and accident analysis tool for NGNP. SPECTRA analyses, some of which have been discussed above, will be used to make code comparisons and inform MELCOR modeling and development for NGNP. The present development status of DAMD is not known, but in the event the code or results are available, they may be informative as a point of comparison, as with SPECTRA. The ECART code is freely available, and may be downloaded from the internet. 
Appendix C

\section{Vendor DDN/PIRT Reconciliation}




\section{Appendix C-Vendor DDN/PIRT reconciliation}

The issues raised in the fission product transport Phenomena Identification and Ranking Table (PIRT) have been review by AREVA, General Atomics, and Westinghouse and reconciled with their established Design Data Needs (DDNs). Dust related issues from these reports are summarized in this section.

\section{C-1. Areva}

A summary of the AREVA DDN/PIRT reconciliation for dust issues is shown in Table C-1. Most were covered by existing DDNs; a new DDN was created for dust/fission product interaction, which was determined to need further study.

Table C-1. AREVA DDN/PRIT reconciliation for dust issues (AREVA 2008).

\begin{tabular}{|l|l|l|l|}
\hline \multicolumn{1}{|c|}{ Issue } & \multicolumn{1}{c|}{$\begin{array}{c}\text { AREVA DDN } \\
\text { No. }\end{array}$} & \multicolumn{1}{c|}{ DDN Title } & \multicolumn{1}{c|}{ Notes } \\
\hline $\begin{array}{l}\text { Dust distribution under } \\
\text { normal operation }\end{array}$ & N/A & N/A & $\begin{array}{l}\text { Issue will be resolved in normal } \\
\text { design work. Method validation } \\
\text { supported by DDNs described } \\
\text { elsewhere. }\end{array}$ \\
\hline Dust generation & 2.4 .1 .0 & Graphite & Existing DDN \\
\hline (De)Absorption on dust & 2.4 .2 .0 & $\begin{array}{l}\text { Graphite/fission product } \\
\text { (FP) interactions }\end{array}$ & New DDN \\
\hline Aerosol growth & 4.1 .4 .1 & FP Transport & Modified DDN \\
\hline Aerosol/dust deposition & 4.1 .4 .1 & FP Transport & Modified DDN \\
\hline $\begin{array}{l}\text { Aerosol/dust bounce, } \\
\text { breakup during deposition }\end{array}$ & 4.1 .4 .1 & FP Transport & Modified DDN \\
\hline Resuspension & 4.1 .4 .1 & FP Transport & Modified DDN \\
\hline $\begin{array}{l}\text { Combustion of dust in } \\
\text { confinement }\end{array}$ & N/A & N/A & $\begin{array}{l}\text { Issue will be resolved in normal } \\
\text { design work }\end{array}$ \\
\hline
\end{tabular}

\section{C-2. Westinghouse}

Westinghouse comments on PIRT issues and their relevance to pebble bed modular reactors (PBMRs) are given in Table C-2. Most issues were deemed resolved by analyses in support of the South African demonstration power plant (DPP). Analyses appear to have been largely based on AVR data and DAMD analysis. Dust breakup was not present in the table. 
Table C-2. Westinghouse DDN/PIRT reconciliation for dust issues (Westinghouse 2009).

\begin{tabular}{|c|c|}
\hline Issue & Notes \\
\hline $\begin{array}{l}\text { Dust distribution under } \\
\text { normal operation }\end{array}$ & $\begin{array}{l}\text { Modeled with AVR data in DAMD to cover all deposition mechanisms in an } \\
\text { integrated manner by an empirical treatment }\end{array}$ \\
\hline Dust generation & $\begin{array}{l}\text { Data available from AVR/Thorium Hochtemperatur Reaktor (THTR). DEACO } \\
\text { characterizing AVR piping sections in terms of deposited dust particle size and } \\
\text { radionuclide inventory. Bounding analyses in dust generation evaluation } \\
\text { utilized to cover uncertainties. Additional dust generation tests under } \\
\text { evaluation for DPP. }\end{array}$ \\
\hline (De)Absorp & $\begin{array}{l}\text { DAMD calculates based on AVR experience the transport of FPs to and from } \\
\text { the dust on the fuel spheres. Analyses include the FPs on dust, on the spheres, } \\
\text { and on the dust on the spheres, as well as on the metallic components within } \\
\text { the HPB. Benchmarked with AVR/THTR experience as well as from } \\
\text { VAMPYRE tests. Location of FPs whether on dust or on surfaces is important. }\end{array}$ \\
\hline Aerosol growth & $\begin{array}{l}\text { Aerosol growth considered in RB with ASTEC. AVR particle size distribution } \\
\text { initially assumed. Industry standards used for particulate behavior. }\end{array}$ \\
\hline Aerosol/dust deposition & $\begin{array}{l}\text { In HPB, modeled with AVR data in DAMD to cover all deposition } \\
\text { mechanisms in an integrated manner by an empirical treatment in RB, modeled } \\
\text { with ASTEC using industry standard aerosol transport algorithm }\end{array}$ \\
\hline $\begin{array}{l}\text { Aerosol/dust bounce, } \\
\text { breakup during } \\
\text { deposition }\end{array}$ & $?$ \\
\hline Resuspension & $\begin{array}{l}\text { Modeled by shear force ratio based on conservative data base from TECDOC } \\
\text { 978. NRG developing SPECTRA model to complement other PBMR codes. } \\
\text { Further, an integrated code DAMD for PBMR under development. CIEM } \\
\text { (Spain) performing literature review. }\end{array}$ \\
\hline $\begin{array}{l}\text { Combustion of dust in } \\
\text { confinement }\end{array}$ & $\begin{array}{l}\text { Study of dust combustion in reactor building performed by UK consultant } \\
\text { indicated no combustion }\end{array}$ \\
\hline
\end{tabular}

\section{C-3. General Atomics}

Most dust issues for the General Atomics prismatic design have been covered by existing DDNs (Table C-3). The general conclusion is that dust effects are not significant for a prismatic reactor. Conservative estimates for dust quantities and properties for a prismatic reactor have been given previously. 
Table C-3. General Atomics DDN/PIRT reconciliation for dust issues (General Atomics 2008).

\begin{tabular}{|c|c|c|c|}
\hline Issue & GA DDN No. & DDN Title & Notes \\
\hline $\begin{array}{l}\text { Dust distribution } \\
\text { under normal } \\
\text { operation }\end{array}$ & $\begin{array}{l}\text { C.07.03.07 } \\
\text { C.07.03.11 } \\
\text { C.07.03.16 }\end{array}$ & $\begin{array}{l}\text { Radionuclide Deposition } \\
\text { Characteristics of Structural Materials } \\
\text { Characterization of the Effects of Dust } \\
\text { on Radionuclide Transport Plateout } \\
\text { Distribution Validation Data }\end{array}$ & $\begin{array}{l}\text { Dust effects of minimal } \\
\text { importance with prismatic } \\
\text { core. }\end{array}$ \\
\hline Dust generation & C.07.03.11 & $\begin{array}{l}\text { Characterization of the Effects of Dust } \\
\text { on Radionuclide Transport }\end{array}$ & $\begin{array}{l}\text { Dust effects of minimal } \\
\text { importance with prismatic } \\
\text { core. } \\
\text { Confirmed by Fort St. Vrain } \\
\text { (FSV) and High Temperature } \\
\text { Engineering Test Reactor } \\
\text { (HTTR) data. }\end{array}$ \\
\hline $\begin{array}{l}\text { (De)Absorption } \\
\text { on dust }\end{array}$ & $\begin{array}{l}\text { C.07.03.07 } \\
\text { C.07.03.11 } \\
\text { C.07.03.16 }\end{array}$ & $\begin{array}{l}\text { Radionuclide Deposition } \\
\text { Characteristics of Structural Materials } \\
\text { Characterization of the Effects of Dust } \\
\text { on Radionuclide Transport Plateout } \\
\text { Distribution Validation Data }\end{array}$ & $\begin{array}{l}\text { Dust effects of minimal } \\
\text { importance with prismatic } \\
\text { core. }\end{array}$ \\
\hline Aerosol growth & N.07.03.19 & $\begin{array}{l}\text { Physical and Chemical Forms of RNs } \\
\text { Released during Core Heatup }\end{array}$ & $\begin{array}{l}\text { No aerosol formation } \\
\text { expected; radionuclide } \\
\text { concentrations too low even } \\
\text { during core heatup accidents. }\end{array}$ \\
\hline $\begin{array}{l}\text { Aerosol/dust } \\
\text { deposition }\end{array}$ & C.07.03.11 & $\begin{array}{l}\text { Characterization of the Effects of Dust } \\
\text { on Radionuclide Transport }\end{array}$ & $\begin{array}{l}\text { Dust effects of minimal } \\
\text { importance with prismatic } \\
\text { core. } \\
\text { Confirmed by FSV and } \\
\text { HTTR data. }\end{array}$ \\
\hline $\begin{array}{l}\text { Aerosol/dust } \\
\text { bounce, breakup } \\
\text { during deposition }\end{array}$ & C.07.03.11 & $\begin{array}{l}\text { Characterization of the Effects of Dust } \\
\text { on Radionuclide Transport }\end{array}$ & $\begin{array}{l}\text { Dust effects of minimal } \\
\text { importance with prismatic } \\
\text { core. } \\
\text { Confirmed by FSV and } \\
\text { HTTR data. } \\
\end{array}$ \\
\hline Resuspension & $\begin{array}{l}\text { C.07.03.09 } \\
\text { C.07.03.10 } \\
\text { C.07.03.17 } \\
\text { C.07.03.18 }\end{array}$ & $\begin{array}{l}\text { Radionuclide Re-entrainment } \\
\text { Characteristics for Dry } \\
\text { Depressurization } \\
\text { Radionuclide Removal Characteristics } \\
\text { for Wet Depressurization } \\
\text { Radionuclide "Liftoff" Validation Data } \\
\text { Radionuclide "Washoff" Validation } \\
\text { Data }\end{array}$ & $\begin{array}{l}\text { Radionuclide re-entrainment } \\
\text { under dry and water ingress } \\
\text { conditions to be } \\
\text { characterized. }\end{array}$ \\
\hline $\begin{array}{l}\text { Combustion of } \\
\text { dust in } \\
\text { confinement }\end{array}$ & N/A & & $\begin{array}{l}\text { Dust effects of minimal } \\
\text { importance with prismatic } \\
\text { core. Confirmed by FSV and } \\
\text { HTTR data. }\end{array}$ \\
\hline
\end{tabular}


Appendix D

\section{Proceedings of the VHTR Dust Assessment Meeting}




\section{Appendix D-Proceedings of the VHTR Dust Assessment Meeting}

\section{D-1. Introduction}

Carbonaceous dust is present in the coolant of high temperature gas-cooled reactors (HTGRs). It is generated through abrasion, chemical reactions, and spallation of friable surface films. Some fission products diffuse from the fuel matrix and adhere to the dust particles. The radioactive dust particles are then deposited throughout the cooling system and can be remobilized into the reactor building in the event of a pipe break. Dust, therefore, may have an impact on worker dose rates and mechanistic source term.

There is large uncertainty with regard to the generation, transport, deposition, and interaction of dust with fission products and components of the reactor system. Some data is available from past HTGR programs but considerable gaps remain and the extent to which the existing data is relevant to modern HTGR designs is unknown.

The issue of dust in HTGRs overlaps many areas of research, including fission product migration through graphite, fluid transport, chemistry, tribology, and materials. Consequently, a comprehensive treatment is not found in the separate Next Generation Nuclear Plant (NGNP) Research and Development (R\&D) planning documents (roadmaps and technical program plans). Although some facet of dust is discussed in each of these documents, an integrated approach is needed to identify the knowledge gaps and prioritize any research needed to fill those gaps. The NGNP Project therefore hosted informal discussions among knowledgeable parties that led to the formal assessment meeting summarized in this document. The first informal discussion took place in Washington, D.C. on November 19, 2009. Presentations given at that meeting by academic, laboratory, and industry personnel provided a brief overview of current research activities and major issues. A pair of meetings followed in late April of 2010 in conjunction with the Very High Temperature Reactor (VHTR) Annual Review. These meetings focused on the ability to model dust transport and fission product interactions, even more specifically on the current and planned capabilities of the MELCOR code with regard to dust and fission product behavior. Work on the MELCOR code has been underway at Idaho National Laboratory (INL) and Sandia National Laboratory. Those development teams have since coordinated their efforts.

The objective of the March 2011 VHTR Dust Assessment Meeting was to survey the state of knowledge in all aspects of HTGR dust and identify the R\&D that needs to be addressed by either the NGNP Project or its collaborators. The discussion notes will be used to draft a review of VHTR dust safety issues (a preliminary review was issued by INL in advance of the assessment) and to enhance or supplement the Fuel Qualification, Graphite, and High Temperature Materials Qualification, and Methods Technical Program Plans.

All documents associated with the informal discussion meetings and this assessment, including the Preliminary VHTR Dust Safety Issues review presented in this document, were posted to a public website for convenient access by the participants (URL: https://secure.inl.gov/dust2010/workshop2011.aspx). 


\section{D-2. Format and Agenda of the VHTR Dust Issues Assessment}

The agenda for the VHTR Dust Issues Assessment is presented below.

\section{Organizers}

Hans Gougar (Chair), Paul Humrickhouse (Idaho National Laboratory)

Sudhamay Basu (Nuclear Regulatory Commission)

Madeline Feltus (Department of Energy)

\section{Format}

The meeting was divided into four successive technical sessions covering: Generation and Characterization, Transport, Fission Product Interactions, and Combustion and Other Issues. A few experts were invited to serve as panel members. Each session began with an introductory presentation given by the Lead Panelist followed by briefings from the other panel members. The briefings and discussion focused upon:

- physical phenomena and impact on HTR safety and licensing

- computational modeling and code maturity,

- availability and suitability of input and validation data (gap analysis)

- current and planned experiments

- impact upon licensing

The final session served to summarize major findings of the earlier sessions and formulate R\&D needs.

\section{Moderators}

Dust Generation: Characterization, Shape Factors (Don Carlson, NRC)

Transport Processes: Deposition, Breakup, Resuspension, Electrostatics (Paul Humrickhouse, INL)

Fission Product Interaction (Sudhamay Basu, NRC)

Combustion, Ingress, and Other Issues (Rob Bratton, INL)

\section{Agenda}

Monday, March 14

08:00 Welcome

08:30 Introductions and Opening Remarks

09:00 Session I: Dust Generation, Characterization, Shape Factors

12:30 Lunch No Host

13:30 Session II: Transport Processes (Deposition, Breakup, Resuspension, Electrostatics)

Tuesday, March 15

08:00 Convene

08:30 Session III: Fission Product Interactions

12:00 Working Lunch: Continuation of Session III Provided

13:30 Session IV: Combustion, Ingress, and Other Issues

Wednesday, March 16

08:00 Convene

08:30 Session V: Summary and Follow-on Activities 


\section{D-3. Technical Discussions}

This section summarizes the major elements and findings of the technical sessions.

\section{D-3.1 Generation, Characterization}

\section{D-3.1.1 Opening Remarks and Questions for the Panel}

\section{Context and Perspective}

Why is dust important? It is relevant in that it affects mechanistic source term, but there are probably other mechanistic source term-related issues of higher importance.

Missouri Studies on High Purity Graphite (GRAFTEC) - S. Loyalka

Spark Generation: produces particles 10-200 nm, carbon particles are not spherical, asymmetry in charge distribution

Abrasion: Dust particle sizes range from 18 to $800 \mathrm{~nm}$ with a wide range of shapes. Very high surface area per unit mass. $68 \%$ porosity, with pore diameter $10-60$ angstrom. This probably leads to high fission product retention.

The shape and size profile varies significantly for different grades and A3-3 (pebble) carbon composite.

Operating Reactor Experience - D. Hittner

Mainly Arbeitsgemeinschaft VersuchsReaktor (AVR), some from High Temperature Test Reactor (HTTR).

Based on studies at the research center in Jülich, Germany, friction is the most important source of dust in a pebble bed reactor (PBR). This is consistent with the observation that far more dust is generated in PBRs than in prismatic reactors. PBR dust is formed mainly in the fuel handling system (pebbles scraping on the metal tubes), not from pebble motion. This has been confirmed by researchers at the Institute for Nuclear Energy Technology (INET) in China.

Some corrosion of graphite in the AVR and Thorium HochTemperatur Reaktor (THTR) has been associated with ingress of different substances (air, water, oil). Carbon deposits may result from reverse boudouard reaction.

Fast transient: increasing the circulator speed in AVR caused dust concentrations to spike. Most of the dust was deposited in hard crust on internal walls, independent of generation mechanism. Dust crust did not easily break loose. Very little loose dust was found.

Questions:

1) Was the hard crust common to high temperature reactors (HTRs) or specific to AVR?

2) Will it remain immobilized under all conditions?

3) Is dust production dependent on chemistry? If so, why the differences between prismatic and PBRs? We must understand the chemistry.

4) How can the design be changed to mitigate dust generation?

Carbonaceous layers were also observed In the Peach Bottom (prismatic) reactor but they were dependent upon the type and temperature of the metal upon which they were deposited. 


\section{Dust Generation - P. Humrickhouse}

Conventional wisdom:

- Dust is a PBR issue (abrasion) and not a problem in prismatics. Wear mechanisms are adhesion, abrasion, fatigue, and corrosion. Wear coefficients are temperature-dependent but not uniformly so. Wear coefficients are also dependent upon the chemistry of the coolant and the impurities in the metals.

- Dust generation behavior is similar in the HTR-10.

- Three to $5 \mathrm{~kg} /$ year in AVR produced in reverse catalytic reaction with metal in steam generator. This, not abrasion, was the source of most dust in AVR according to R. Nieder, but this is not a majority opinion, even at Jülich.

Question: Can wear coefficients be obtained for A3-3 given that it is a composite of graphite, binder, etc.?

Check AGC-1 heatup results versus AGC-2. AGC-1 uses A3-3 binder.

\section{Dust Generation and Combustion - A. Tokuhiro}

The University of Idaho is engaged in a research project in which stress calculations and machined graphite (SGL) sphere-on-sphere wear measurements are being performed. However, the project is not yet performing measurements of wear between A3-3 pebbles, pebbles and graphite, and pebbles and metals.

\section{Resuspension - G. Poss}

Concerning source term the retention factor of the reactor confinement is essential. The resuspendable fraction of fission product loaded graphite dust in the primary circuit, depending on the dominant accident scenarios (e.g. steam generator tube rupture, subsequent loss of coolant via stuck-open safety valve), should be chosen as basis for mechanistic modeling of the fission product transport and deposition phenomena in the confinement building. Making experiments as simple as possible is highly recommended to validate CFD and LP models. This covers multicompartment configurations, dead end zones etc.

Graphite Wear in HTR-10 - S. Yu

Generation: Mechanical wear-pebble-on-pebble and pebble in fuel handling systems (FHS):

- Roughness is important

- INET experiments show that wear mass loss rate in helium is much greater than in air or nitrogen.

- INET experiments with pebbles in tubes show that gas flow prevents the formation of a lubricating layer and the flow also affects the collision frequency.

HTR-10 result: $15 \mathrm{mg}$ mass loss per cycle and $0.07 \mathrm{~kg} / \mathrm{MW}(\mathrm{t}) /$ year. Compare to AVR in which 0.1 $\mathrm{kg} / \mathrm{MW}(\mathrm{t}) /$ year was formed and International Atomic Energy Agency reports, which indicate that $0.05 \mathrm{~kg} / \mathrm{MW}(\mathrm{t}) /$ year was formed in THTR

Other investigations underway at INET (HTR-1):

- Different (less rough) materials for FHS tubes

- Ways to decrease the tube length

- Dynamics of pebbles in tubes

- Effect of gas flow 
- $\quad$ Coating pebbles with SiC. Need more data. Should the tubes be coated as well? Will these coatings spall and generate more dust?

Simulations: Need a set of dimensionless parameters that characterize dust generation.

Other INET questions:

1) Is dust generation affected by the fuel loading in pebble?

2) Does irradiation change the surface area and porosity of pebbles? Probably.

Phenomena and processes responsible for the generation of dust in a gas-cooled reactor include:

- Abrasion and impact both within the core and elsewhere, including within any fuel handling system.

- Disproportionate reactions, including:

$-\mathrm{C}_{\mathrm{n}}$ (gas) $\rightarrow \mathrm{C}$ (solid) $\mathrm{n}=1,2,3$

$-2 \mathrm{CO} \rightarrow \mathrm{CO}_{2}+\mathrm{C}$ (solid).

- Neutron irradiation of graphite and stress relief of graphite that accumulates radiation damage (probably not significant at expected fluence levels).

- Rust dust formed by spallation of oxides and carbides from metal surfaces or even the abrasion of fines from metal surfaces.

Most of the discussion at the meeting was on the first two processes: abrasion of matrix and graphite structures, and gas-to-particle conversion. Data from past gas-cooled reactors, such as AVR, appear to suggest that abrasion is the predominant source. However, some have suggested that gas-to-particle conversion would become a more predominant source as engineering efforts to reduce abrasion reached fruition. Gas-to-particle conversion comes about because of impurities (air, water vapor) in the coolant (helium) reacting with graphite to form carbon monoxide.

The possibility of dust generation due to neutron irradiation was mentioned, but the potential magnitude of this source is not known. Rust dust was mentioned at the meeting but not discussed. Until there is specification of the metal alloys to be used in the reactor, about the only thing that can be said about this source is that metal oxidation will occur in the presence of water.

Current investigations of dust generation include:

- Abrasion mechanism to support engineering efforts to reduce abrasion (University of Idaho)

- Abrasion and gas-to-particle conversion (University of Missouri)

- Abrasion mechanism (China).

Current inpile experiments will be conducted to see if neutron irradiation can be a significant source of dust. It may also be that irradiation will roughen surfaces so that more dust is generated by abrasion.

University of Missouri work showed that particles produced by gas-to-particle conversion are chain agglomerates of essentially spherical primary particles. These particles can potentially have very large shape factors $(>10)$ and very high surface areas for radionuclide absorption. Particles produced by abrasion are not spherical but can have modest shape factors $(<4)$. They have a pore structure with pore diameters of $\sim 50$ Angstroms, which complicates prediction of radionuclide absorption. It remains to be demonstrated that this is an issue in HTGRs.

There do not appear to be predictive models. There was some discussion that the PEBBLES code attempted to predict dust generation rates, but it is unclear that this is a mechanistic model suitable for generic application to gas-cooled reactors. On the other hand, it may not be essential to have precise 
predictive models. It is essential to know that there are different types of dust. It may be that accident analyses have to be done assuming a broad range of dust inventories and a range of contributions by dusts of different types. It will be important to know the aerosol characteristics of dust generated by neutron irradiation if this mechanism is, in fact, an important source of dust.

\section{D-3.2 Transport}

The Transport session covered the mechanisms of dust transport. There is a general consensus that dust particles are not spherical but can reasonably be assumed to be formed of spherical subparticles. This assumption will be helpful in modeling efforts.

Deposition - T. Lind

Charging: Charging of dust particles increases deposition. Particles are self-charged but can be neutralized by ions. Charging may be important in confined geometries.

Impaction: Particle bounce and breakup by impaction are areas that must be studied further.

Question: Are particles more or less likely to deposit on a fresh surface?

Resuspension: One cannot compute resuspension with today's models because it is sensitive to many factors. At least part of the AVR dust could be resuspended (Moormann).

Deposit Aging: This phenomenon is strongly linked to resuspension. It is uncertain how AVR dust became a crust that formed on the insides of pipes but is postulated that water and oil ingress play a role.

We do not know the:

- Dust generation rate

- $\quad$ Size and morphology (transport and breakup)

- Electrostatic effects

- $\quad$ Adhesive forces

- Complex geometries and flow field

- Effect of changes in the substrate that may also lead to resuspension.

Resuspension - F. Parozzi

Resuspension issues include:

- Aerodynamic forces can stop deposition. Traditional aerosol deposition models neglect resuspension and thus are not valid.

- $\quad$ Dry particles already deposited can form aggregates and still be resuspended.

- Conservation laws do not improve resuspension models.

- ECART semiempirical approach seems to work well. Mechanistic approaches require too many tuning factors.

- Resuspension depends upon thickness of deposit. Drag forces dominate lift forces.

- Aerosols do not resuspend in the presence of moisture.

- Resuspension is complex and perhaps chaotic.

Resuspension model requirements are: 
- Wide applicability

- Reasonable running time

- Must work for pipes, vessels, and heat exchangers

- Physical-chemical data and accommodation factors as input

- Accuracy of model must be consistent with accuracy of boundary conditions

- Phenomena computed must be measurable and visible.

Dust Effects in Prismatic HTRs - D. Hanson

Important phenomena are not aerosol and dust transport but fission product release rates and speciation. General Atomics deliberately assumed design margins of $4 \mathrm{x}$ for fission gases, $10 \mathrm{x}$ for fission metals, and 10x for plateout/liftoff to get within an order of magnitude. We are not there yet.

Impact of dust:

- In HTGRs, there is been minimal impact except for pebbles damaged by control rods in THTR:

- Peach Bottom: lots of soot from oil, no real impact

- Fort St. Vrain: dust was mostly rust (not carbonaceous), no real impact

- In non-nuclear high temperature gas systems, dust is only an issue at very high dust loadings in refineries. As the concentrations and chemistry are significantly different, this is likely to be irrelevant to HTGRs.

- Dust is likely a $2^{\text {nd }}$ or $3^{\text {rd }}$ order effect on any phenomena.

Needs include:

- Sorption isotherms for fission product in primary - an empirical model compared against COMEDIE tests got within an order of magnitude.

- For dust:

- Separate effects and an inpile loop testing should be performed

- Integrated accident and irradiation behavior data

- Start with clean (no dust) case.

- Validate the fission product transport codes that exist. Dust is not explicitly modeled. Design margins should cover the uncertainty imposed by dust. Current methods are sufficient for conceptual and preliminary designs. Final design requires more rigorous validation and reduction of uncertainty. Proof is in the prediction of integral test results for fission product transport.

Aerosol Interactions and Dynamics - S. Loyalka

Aerosol transport is reasonably captured with MAEROS module in MELCOR.

Dust transport studies at the University of Missouri are as follows:

- Thermophoresis is postulated as an important deposition mechanism AVR, but this needs to be confirmed. Current work shows that a very fine mesh is needed to model the thermophoresis boundary layer.

- Resuspension (adhesion and modeling)

- Looking at composition and charge effects not captured by the sectional technique of MELCOR Experimental Data Needs of System Codes - B. Winters \& M. Stempniewicz 
System codes require the following experimental data:

- Dust generation

- Dust transport and deposition needs/gaps:

- Influence of wall roughness

- Pipe entrance effects

- Impaction at pipe bends

- Stagnation (dead) zones

- High flow zones

- Resuspension needs:

- Large uncertainty in adhesion forces; measure these then validate models. This may prove to be too difficult without knowing the precise nature of dust and the surface state of the primary circuit metals.

- Confinement/containment behavior

- Other probably minor phenomena:

- Effect of dust cloud on heat transfer from reactor pressure vessel

- Data on impaction of dust on cavity walls

- Estimation of importance of different phenomena - (see the presentation for details).

\section{MELCOR Capabilities - M. Young}

MELCOR has the following capabilities:

- Agglomeration and deposition of aerosols, no turbulent deposition

- Modeling thermophoresis, although this is probably not a significant phenomenon in HTGRs

- The code needs size distribution of deposited aerosols - aerosol growth at low dust concentrations

- Current resuspension models not well developed — not much data exists

- fission product adsorption

- Condensation of fission product on aerosols

- Chemisorption of cesium

- Deposited dust will cover part of wall surface, competing for fission products - well understood

- Electrostatics?

- Breakup upon impact is probably minor.

Dust generated by whatever mechanism will enter into the coolant flow within the reactor coolant system. This circulating dust will deposit on surfaces. Little dust is expected to be circulating in the coolant system at any one time during normal operations.

There do not appear to be any qualitative differences in the transport and deposition of dust than in usual aerosol physics. Quantitative differences arise because of differences in particle properties (size, shape factors, density, and the like). For the purposes of accident analysis, the resuspension of deposited dust is the topic of interest. 
Crusting and bonding of deposited dust to surfaces in the AVR might be the result of lubricating oil intrusion into the reactor and subsequent pyrolysis or sintering, but the formation mechanism is not really known. Dust formed from oil ingress was observed in Peach Bottom.

Deposited particles can be resuspended by a variety of mechanisms including:

- Lift from surfaces by increases in flow velocity

- Turbulent jets penetrating to surfaces from the bulk flow

- Shock and vibration of the deposit substrate

- Impact of circulating particles on particles already deposited on surfaces.

Planned Investigation of Resuspension

1) Work planned in connection with the DUSTIN project in Europe. The investigations seem to be exclusively focused on resuspension caused by changes in flow. There is a willingness to examine the effects of substrate shock and vibration (which will occur under accident conditions) if estimates of frequencies of vibrations and amplitudes can be supplied. There are computational tools that could provide such estimates.

2) Two national experimental projects -STAUB-II (Prof. Hurtado, Dresden) and TARGET (Prof. Allelein, Aachen/Jülich)- planned in Germany.

\section{D-3.3 Fission Product Interactions}

Opening Presentation - S. Basu

Questions:

1) What are the physical phenomena?

2) Which are important for licensing (safety-significant):

- fission product adsorption

- clustering

- fission product transport

- fission product retention and release

- effects of moisture and air ingress?

Questions to be answered?

1) Do dust-free (clean) scenarios provide an upper-bound estimate of fission product release?

2) Does dust promote or inhibit fission product retention?

3) How are these affected by moisture?

4) How much dust is a significant amount?

5) Why care about dust?

Dust is a mechanism for getting radiological inventory quickly into the containment (D. Powers).

Dust concentrations are normally low.

Graphite adsorption varies widely by graphite type (surface area) - can fit data to a Langmuir isotherm, and is affected by irradiation - vacancy formation and formation of functional groups on displaced planes. 
Effects of water vapor:

- Surfaces have varying levels of polarity

- Functional groups on basal planes are also active sites for water absorption.

Technical framework for radionuclide movement in the core exists (dusty gas model) that can handle air and water interactions. Needs include:

- Characterize irradiated graphite (permeability and porosity; and put it in the spec?)

- Characterize dust and dust deposits

- Develop an empirical database on vapor isotherms.

Fission product experiments - D. Hanson

Experiments are often not protypical. A predictive capability is needed as long as it is predicated upon simpler reproducible experiments. Baseline experiments must be dust-free, so dust can be added to see its effects. Need a way to extract data from HTTR and HTR-10. Past reactors are not comparable to modern designs (oil ingress, control rods into core, etc.)

Dust effects probably need not be modeled for prismatic reactors. Under current regulations, vendors do not take credit for hold-up in the building except perhaps during a depressurized conduction cooldown (Modular High Temperature Gas Reactor Preliminary Safety Information Document).

Fission product Interactions - S. Loyalka

A hindered diffusion model can be used, Langmuir. The deposit rate on chain-like particles has been computed.

Experiments and validation of system codes - F. Parozzi

Aerosol models are partially validated and should address:

- resuspension

- chemical interactions - equilibrium assumed

- multicomponent behavior

- interaction with radiation field

- $\quad$ effects of particle shape.

Thoughts in response to a question from Basu - Poss

- Assuming that the entire FP inventory released from fuel would find its way into the environment (extremely conservative), the dust free scenario would indeed provide an upper bound estimate of FP release.

- Under the presence of dust the amount of FP released into the confinement and into the environment might be less than the supposedly upper bound estimate but there might be situations which quantitatively come close to the upper case. Time constants might be different in both cases.

Whether in the presence of graphite dust, fractions of FPs might mix with the circulating dust depends on the properties of the interacting species (graphite dust/FP's). FPs might be trapped irreversibly or might be chemically or physically sorbed and released again depending on TH conditions.

- It cannot be excluded that in presence of graphite dust, FP inventory might have a preferential adherence to circulating dust and that the FP inventory will be released. The release will only be accelerated in time as dust will act as an enhanced transport medium 
- Concerning the post examinations of AVR tubes (crusts, dust fixed) there might be doubts about their representativeness. The observed integral results might be the outcome of many incidents during AVR lifetime.

- It has been shown that the dominant aerosol term is caused by mechanical vibrations, followed by the processes of flow reversal and re-entrainment by enhanced gas flow. For water ingress scenarios, a substantial aerosol source is caused by graphite corrosion.

Fission Product Sorption - I. Szlufarska

Graphite becomes more sorptive after irradiation.

More graphitization leads to less sorption (less surface area and the chemical nature of the surface is altered). However, recent results show the opposite behavior. Why?

BET measurements of surfaces are less accurate for more impregnated material.

Questions:

1) Would another porosity measurement technique better characterize the surface?

2) How does cesium sorption affect that of other species?

3) We must quantify the impurity level.

Dust created within the reactor will become contaminated with absorbed fission products. Dust created by abrasion may be contaminated when it is first formed. The contamination comes from the operational releases of fission products out of defective or failed fuel. Similarly, radionuclide releases during normal operations will create a partial pressure of fission product vapors that can be gettered by the dust. Anticipated fission product vapor concentrations are not expected to be high enough to form a fission product aerosol to interact with the dust. The contamination will be carried into the containment by any dust mobilized (resuspended) during depressurization associated with an accident.

The principal phenomena of interest are:

1) Fission product absorption on dust. Fission product vapors will absorb on active sites on dust. The absorption is characterized by "isotherms" peculiar to each radionuclide and each type of dust. At present, there does not appear to be technology to predict either the isotherm for any radionuclide or the total concentration of active sites for any particular graphite.

2) Fission product absorption on graphite and metal surfaces. Fission product vapors can also absorb on graphite structures and metal surfaces in the coolant system. Radionuclides absorbed on these surfaces will not be promptly dispersed into the reactor containment during a blowdown the way fission products are on dust. There is, then, a competition for dust between surfaces and dust that will have to be evaluated in an accident analysis.

Some investigations of absorption isotherms are being conducted at the University of Wisconsin. Powers is collecting isotherms for various graphites. There does not seem to be a systematic investigation of absorption isotherms for the particular graphites and dust to be used in the planned gas-cooled reactors.

3) Active site formation. Dust and graphite will have natural active sites for adsorption as well as pores for absorption. Additional active sites can be formed during irradiation of the graphite. A common mechanism for active site generation is the nucleation of graphite plates by the accumulation of displaced carbon atoms in the interplanar spaces of graphite.

4) Competition for active sites by other species. Other species in the coolant - notably water vapor and carbon monoxide - can also absorb on active sites of graphite and dust. Though these impurities will be present at very low concentrations, they will be at concentrations much higher 
than radionuclide concentrations in the coolant during normal operation or postulated accidents. The impurities in the coolant can effectively compete with radionuclides for active sites on dust and graphite. There is a well-established technology for understanding water vapor absorption on graphite, though it is not evident that isotherms can be predicted for any particular graphite.

Truly predictive models for radionuclide absorption on arbitrary graphites and carbon are beyond the state of the art. It is relatively easy to develop models that apply empirically determined isotherms to prediction of absorption of radionuclides on graphite and dust, even in the face of competitive reactions with metal surfaces and site competition by impurities in the coolant gas. Isotherms can be estimated or bounded for major radionuclides such as iodine, cesium, strontium, and silver.

Accuracies may not be high if accurate estimates of surface area and pore structure are not available.

\section{D-3.4 Combustion and Other Issues}

General Dust Issues - D. Hittner

Concentration of dust in AVR

- Average $=5 \mu \mathrm{g} / \mathrm{m} 3$, range of $1-40 \mathrm{microgram} / \mathrm{m} 3$

- Accident conditions $\sim 1 \mathrm{mg} / \mathrm{m} 3$ peak value when the circulator speed was increased from 1,500 to 4,000 rpm.

Question: Can dust have a significant impact? Answer: No.

\section{Combustion - L. Lommers}

Carbonaceous dust can deflagrate, but the conditions (concentrations, ignition sources, etc.) expected in HTGRs under normal and accident conditions are not expected to sustain combustion in any significant sense. Matrix material (partially graphitized carbon) is the source of dust. Dust agglomerates so its oxidation properties change. Graphite is among the mildest explosives, and its release concentration in a building would be well below explosibility limits. Design changes can mitigate this possibility even further.

The only issue is the quantity of dust and its transport and retention.

\section{Other Issues}

Dust and its heat transfer is not an issue, but dust maintenance and in-service inspection are issues. Dust resuspended during a reactor accident will be injected into an air rich containment atmosphere. The dust will be hot and can react with the atmosphere. Any radionuclides absorbed on the dust may be remobilized by the reaction. Notably, iodine absorbed on dust could be freed in gaseous form.

Dust Combustion. Three modes of dust combustion are detonation, deflagration, and slow oxidation. The detonation of graphite dust suspended in air was the only mode discussed at the meeting. It was generally concluded that the detonation of dust concentrations expected in reactor accidents was not likely. Slower modes of oxidation were not excluded. There do not appear to be any active investigations of dust combustion by either fast modes (detonation and deflagration) or slow oxidation mechanisms. It appears that phenomena associated with slow combustion of dust can be predicted using current modeling supplemented by isotherm models of radionuclide absorption and desorption from dust.

Radionuclide release from oxidizing dust. At expected concentrations, the freed radionuclides would likely remain gaseous, so dust oxidation, whether by fast or slow means, will occur. Agglomeration with residual dust would seem to follow the general processes of aerosol physics as understood for light water reactors, except that shape factors and the like for dust will be very different. There do not seem to be active investigations of the fate of radionuclides when dust oxidizes. 


\section{D-4. Areas of Research}

\section{D-4.1 Dust Generation, Transformation, Resuspension}

Questions include: What is the source of dust; what is its range size, shape, and other properties; why did it form a crust in AVR, and will it do so in modern HTGRs; if so, what are the mechanical properties of the crust that affect resuspension?

University research should be supported to produce data on the characteristics of particles produced by various mechanisms. It would be useful to get dynamic shape factor data on the particles. Also, scour around for data on neutron irradiation as a source of data or a mechanism that can enhance abrasive generation of dust. MELCOR modeling should be upgraded to accept aerosol particles with substantially different shape factors as input, and the code needs to track radionuclides on dust.

It will be useful to stay abreast of work within DUSTIN in the resuspension area and to provide the resuspension researchers useful source materials such as the graphite dust generation research at universities and elsewhere using abrasion and gas-to-particle conversion methods. Estimates of vibrational frequencies and amplitudes for coolant line breaks should be provided and investigators encouraged to look at other mechanisms of resuspension. MELCOR will need a valid resuspension model.

Resuspended particles will be injected into the containment during an accident. The behavior of the particles in containment will depend on the unusual shape factors of these particles. It will be useful to have tests that validate the MELCOR predictions of the dust settling and deposition in a multicompartment containment. Tests that might be useful in this regard are planned in frame of the OECD/NEA THAI 2 project at the THAI facility.

MELCOR should have a good model for fission product absorption on graphites and dust. The model will have to use estimated isotherms until more realistic data are available for actual materials. Isotherm data collection for radionuclides, water vapor, carbon monoxide, and carbon dioxide should continue. Data for fission product absorption on metal surfaces will also need to be available. It is likely that data from isotherms will be for unirradiated material. We will need to have a method to estimate how the isotherms of irradiated material will differ. Accident analyses will have to consider a range of behaviors.

\section{D-4.1.1 Tests to be Performed}

Reactor data:

- Dust filter measurements in HTTR, HTR-10

- Plate-out probes in HTR-10, HTTR

- Crust characteristic data from AVR

- $\quad$ AVR generation rates $(700 \mathrm{~kg}$ total, $20 \mathrm{~kg}$ mobile).

Integral tests:

- Inpile loop plate-out tests with and without dust (clean)

- With water (oiled bearings are not proposed for current designs.

Separate effects tests:

- Tribology and rates of generation:

- Matrix on matrix 
- Matrix on graphite

- Matrix on metal (FHS)

- Irradiated carbon properties

- Particle deposition:

- 'Clean' tests

- With oil/water

- Pipes of different composition and roughness

- Resuspension of crust:

- Mechanical properties (from AVR)

- Generation

- abrasion

- chemical

- catalyzed-oxidation by fission product.

\section{D-4.2 Dust Transport}

How is dust entrained in the coolant during normal and transient conditions? What is the nature of dust deposition and resuspension in stagnation zones? Can one model dust as an aerosol? Yes.

\section{D-4.2.1 Tests to be Performed}

Reactor data:

- Very little information on mobilized dust is available.

- dust spectra can be inferred from filter deposits.

Integral experiments:

- THAI experiments can be used to validate system codes for ex-core dust transport and distribution.

Separate effects tests and fundamental experiments:

- DUSTIN project in Europe will provide a comprehensive investigation of separate effects and fundamental experimental data that can be used as input to and validation of system codes.

- DUSTIN will not examine aging effects; it is difficult to do this experimentally because of the long times involved.

\section{D-4.3 Fission Product Interactions}

What is the fission product loading on dust or crust in the primary and what are its dependencies?

\section{D-4.3.1 Tests to be Performed}

Reactor data:

- Activity measurement in dust in HTTR, HTR-10

- Data from plate-out probes (with and without dust) in HTR-10, HTTR

- Past reactor data must be mined (least expensive) 
Integral experiments:

- characterization of irradiated samples from the AGR graphite experiments.

- Inpile experiments; add dust (graphite, matrix, alloy)

Separate effects tests and fundamental experiments:

- Measure isotherms for fission products and competitive species:

- temperature range

- prototypic metals and dusts (matrix, etc.)

- irradiated dusts

- Measure characteristics of deposited dust

- spatial dependence (variable over sample)

- intra- and extra-dust transport of fission product.

\section{D-4.4 Combustion and Explosibility}

Not a significant phenomena; no R\&D required.

\section{D-5. Follow-up Actions}

1. A brief follow-up meeting was held in Albuquerque, NM in conjunction with the VHTR Annual Review (April 2011) in which the R\&D needs listed above were confirmed and prioritized.

2. NGNP Project personnel must review the vendor design data needs on dust.

3. The Preliminary VHTR Dust Safety Review shall be updated to form a VHTR Dust R\&D Plan to be integrated into the other R\&D planning documents.

4. Department of Energy must establish agreements with the Japan Atomic Energy Agency, INET, and European projects such as DUSTIN to coordinate efforts. 


\section{D-6. Participants}

\begin{tabular}{|c|c|c|c|}
\hline Basu & Sudhamay & NRC & Sudhamay.Basu@nrc.gov \\
\hline Bratton & Rob & INL & robert.bratton@inl.gov \\
\hline Carlson & Donald & $\mathrm{NRC}$ & Donald.Carlson@nrc.gov \\
\hline Caspersson & Sten & Westinghouse & caspersa@westinghouse.com \\
\hline Esmaili & Hossein & NRC & hossein.esmaili@nrc.gov \\
\hline Feltus & Madeline & DOE & Madeline.Feltus@nuclear.energy.gov \\
\hline Gougar & Hans & INL & hans.gougar@inl.gov \\
\hline Hanson & Hanson & General Atomics & David.Hanson@ga.com \\
\hline Hittner & Dominique & Areva & dominique.hittner@areva.com \\
\hline Holbrook & Mark & INL & Mark.Holbrook@inl.gov \\
\hline Humrickhouse & Paul & INL & paul.humrickhouse@inl.gov \\
\hline Kelly & Joe & NRC & joseph.kelly@nrc.gov \\
\hline Lee & Richard & $\mathrm{NRC}$ & richard.lee@nrc.gov \\
\hline Lind & Terttaliisa & PSI & Terttaliisa.lind@psi.ch \\
\hline Lommers & Lew & AREVA & Lewis.lommers@areva.com \\
\hline Loyalka & Sudarshan & U-Missouri & loyalkaS@missouri.edu \\
\hline Merrill & Brad & INL & brad.merrill@inl.gov \\
\hline Nosek & $\mathrm{AJ}$ & $\mathrm{NRC}$ & andrew.nosek@nrc.gov \\
\hline Pappano & Peter & DOE & Peter.pappano@nuclear.energy.gov \\
\hline Parozzi & Flavio & RSE (Italy) & Flavio.Parozzi@rse-web.it \\
\hline Poss & Gerhard & Becker Technologies & poss@,becker-technologies.com \\
\hline Powers & Dana & Sandia & dapower@sandia.gov \\
\hline Rubin & Stuart & $\mathrm{NRC}$ & stuart.rubin@nrc.gov \\
\hline Salpeter & Nathaniel & TAMU & natesal@neo.tamu.edu \\
\hline Scott & Michael & NRC & michael.scott@nrc.gov \\
\hline Srinivasan & Makuteswara & $\mathrm{NRC}$ & makuteswara.srinivasan@nrc.gov \\
\hline Steingass & Timothy & $\mathrm{NRC}$ & txs3@nrc.gov \\
\hline Stempniewicz & Marek & NRG & stempniewicz@,nrg.eu \\
\hline Stummayer & Katharina & GRS & Katharina.stummeyer@grs.de \\
\hline Suh & Namduk & KINS & k220snd@kins.re.kr \\
\hline Szlufarska & Izabela & Uwisc & izabela@engr.wisc.edu \\
\hline Tokuhiro & Akira & U Idaho & tokuhiro@uidaho.edu \\
\hline Wei & Tom & ANL & tycwei@anl.gov \\
\hline Winters & Bert & NRG & winters@,nrg.eu \\
\hline Young & Michael & Sandia & mfyoung@sandia.gov \\
\hline $\mathrm{Yu}$ & Suyuan & INET & suyuan@tsinghua.edu.cn \\
\hline Zaki & Tarek & $\mathrm{NRC}$ & tarek.zaki@nrc.gov \\
\hline Zamore & Janelle & DOE & janelle.zamore@nuclear.energy.gov \\
\hline
\end{tabular}

